\title{
Löslichkeit und Diffusion von Wasserstoff in dünnen Schichten amorpher ZrTiNiCuBe- und ZrAlNiCu-Legierungen
}

\author{
Dissertation \\ zur Erlangung des Doktorgrades \\ der mathematisch-naturwissenschaftlichen Fakultäten \\ der Georg-August-Universität Göttingen
}

vorgelegt von

Joachim Bankmann

aus Diepholz

Göttingen 2002 
Referent:

Korreferent:

Tag der mündlichen Prüfung:
Prof. Dr. R. Kirchheim

Prof. Dr. K. Samwer

Dienstag, 28. Januar 2003 
2.1 BERECHNUNG VON PLATZDichtEVERTEILUNGEN IM SINNE VON ZUSTANDSDICHTEN FÜR WASSERSTOFF IN AMORPHEN LEGIERUNGEN .

2.1.1 Zustandsdichten für den Wasserstoff in Kristallen und binären Legierungen............................ 5

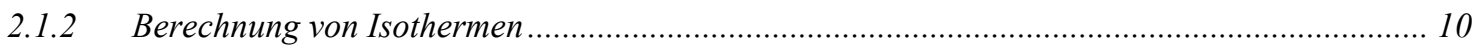

2.2 DIFFUSION VON H IN AMORPHEN LEGIERUNGEN ......................................................................... 12

2.3 ZUSTANDSDICHTEN MEHRKOMPONENTIGER AMORPHER LEGIERUNGEN ........................................... 14

2.3.1 Häufigkeitsverteilung von Tetraederplätzen in einer homogenen amorphen Legierung........... 14

2.3.2 Bestimmung der mittleren Energien der einzelnen Gaußverteilungen ................................. 17

2.3.3 Kurzreichweitige Wechselwirkungen - Blockieren von Tetraederplätzen ............................... 23

3 PROBENHERSTELLUNG UND EXPERIMENTELLE METHODEN..........................................26

3.1 Herstellung Und CharaKterisierung AMORPHER LEGIERUNGSSCHICHTEN AUS ZR - TI - CU -

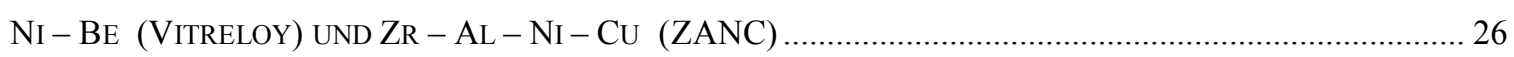

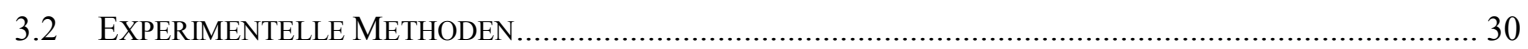

3.2.1 Isotherme elektrolytische Wasserstoffbeladung dünner Schichten........................................ 31

3.2.2 Messung der Wasserstoffdiffusion mit Hilfe der Doppelzelle ...................................................... 33

3.2.3 Ein neues Verfahren zu Bestimmung von Durchbruchszeiten für Diffusionsuntersuchungen.... 35

4 ERGEBNISSE 40

4.1 EXPERIMENTELLE BESTIMMUNG VON ISOTHERMEN UND ZUSTANDSDICHTEN Z(E) , .......................40

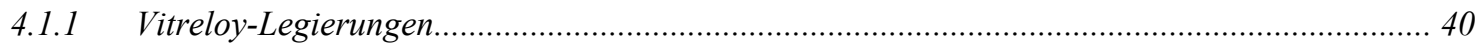

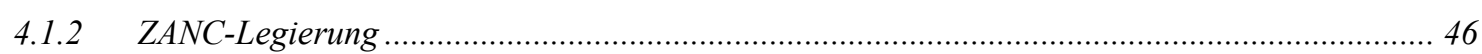

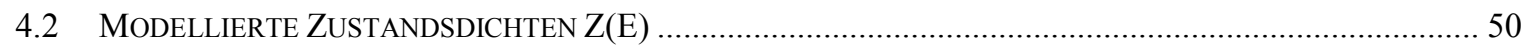

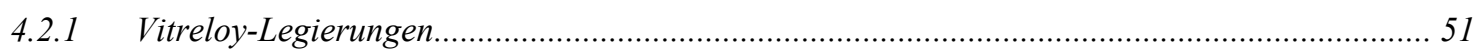

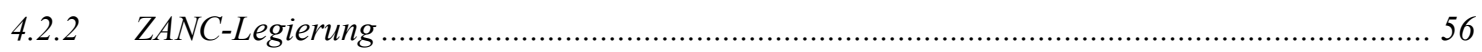

4.3 VERGLEICH DER ZUSTANDSDICHTEN AUS EXPERIMENT UND MODELL .............................................. 59

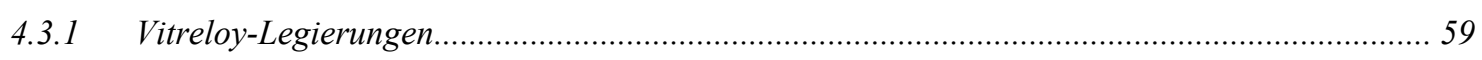

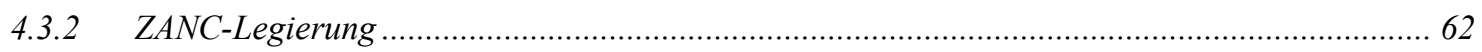

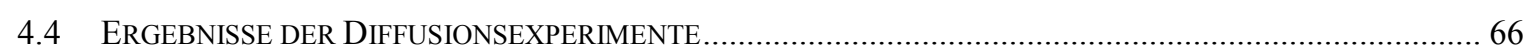

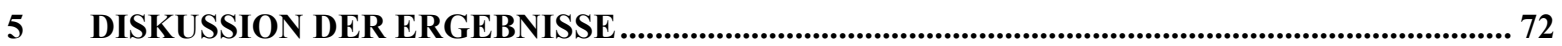

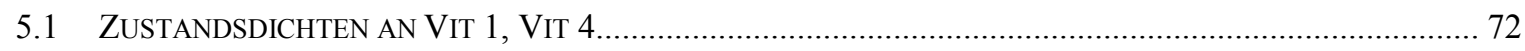

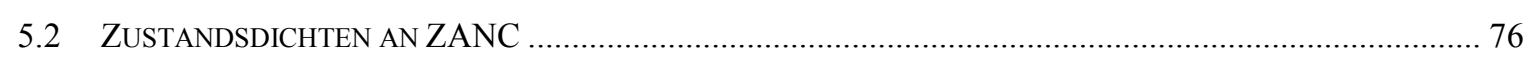

5.3 BESTIMMUNG DER WASSERSTOFFDIFFUSION IN AMORPHEN MATERIALIEN ...................................... 79

5.3.1 Schichtablösung bei der Wasserstoffbeladung ....................................................................... 79

5.3.2 Einfluss der Pd-Deckschicht auf den gemessenen Widerstand .................................................. 80 
5.3.3 Bestimmung des intrinsischen Diffusionskoeffizienten von Wasserstoff in amorphen

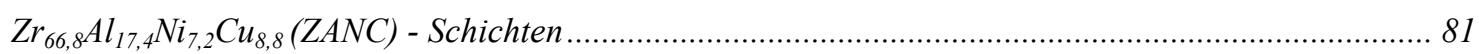

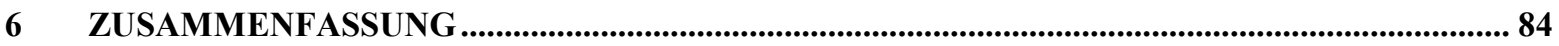

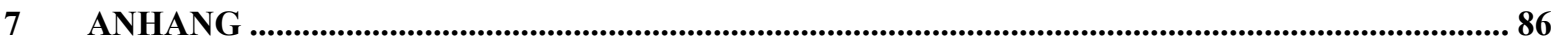

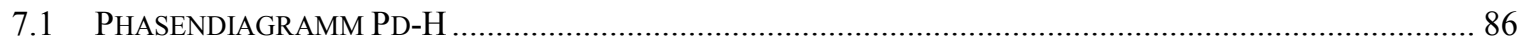

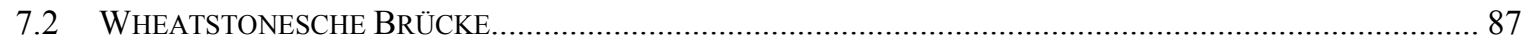

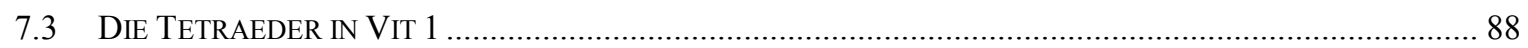

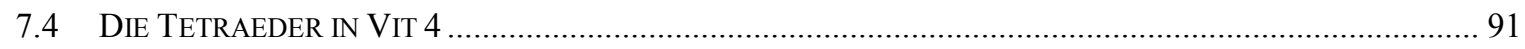

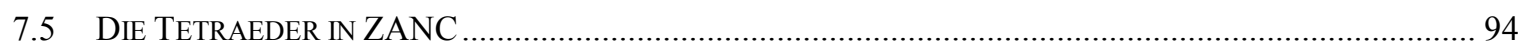

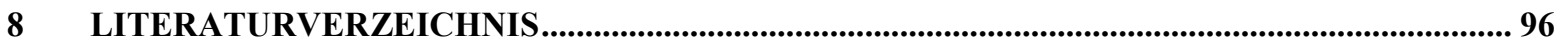




\section{Einleitung}

Seit Graham 1866 [Gra66] entdeckte, dass sich Wasserstoff in nicht geringen Mengen in Palladium löst, ist das Forschungsgebiet "Wasserstoff in Metallen" von sehr großen Interesse. Es gibt seitdem starke Bemühungen, ein fundiertes und umfassendes Wissen über dieses Gebiet $\mathrm{zu}$ gewinnen. Neben den negativen Erscheinungen, wie die Wasserstoffversprödung, die Ursache großer Schäden an metallischen Strukturwerkstoffen verursacht, ist das wirtschaftliche Potential der Wasserstofflöslichkeit von sehr großer Bedeutung. In geeigneten Legierungen, die z.B. auf $\mathrm{LaNi}_{5^{-}}$, NiTi- oder NiZr-Legierungen basieren [Uch91, Yan95, Kie89], lässt sich Wasserstoff in großen Mengen speichern und einfach wieder extrahieren. Mit Wasserstoff als Energieträger hat man damit die Möglichkeit, solche Legierungen als Materialien für Akkumulatoren zur Stromerzeugung einzusetzen. Die heute schon in jedem zweiten Mobiltelefon zum Einsatz kommenden Nickel-Metall-Hydrid (NiMH) Akkumulatoren beweisen die Einsetzbarkeit solcher Metall-Wasserstoffsysteme. Ein zweites großes Forschungsfeld, bei dem solche Systeme eine Rolle spielen, ist die Entwicklung von Brennstoffzellen, die besonders für den Einsatz in Kraftfahrzeugen konzipiert sind, um eine umweltverträgliche Alternative zum herkömmlichen Einsatz fossiler Brennstoffe zu bieten.

Wasserstoff löst sich interstitiell in einem Wirtsgitter und besitzt selbst bei Raumtemperatur noch eine sehr große Beweglichkeit. Dabei kann ein Wasserstoffatom mit einer Vielzahl von Defekten in dem Gitter wechselwirken. Dazu gehören neben Leerstellen und Fremdatomen auch die Segregation von Wasserstoff in Korngrenzen und Versetzungen [Max00]. Die Häufigkeit solcher Defekte hat einen entscheidenden Einfluss auf die Beweglichkeit oder die Löslichkeit von Wasserstoff. Kennt man andererseits die Wechselwirkung zwischen Wasserstoffatom und einem Defekt, so erlauben die leicht messbaren Eigenschaften Diffusion und Druck-Konzentrations-Isothermen Rückschlüsse auf eben diese Defekte. Damit ist der Einsatz von "Wasserstoff als Sonde" [Fla01] möglich, der es zum Beispiel erlaubt Versetzungsdichten oder gar Nahordnungsphänomene innerhalb metallischer Proben zu studieren [Bro89b, Fee88]. 
Amorphe Legierungen zeigen eine große Vielfalt an chemisch und strukturell unterschiedlichen Quasi-Zwischengitterplätzen. Sie sind daher geeignete Systeme, in denen Wasserstoff als Sonde eingesetzt werden kann, um die Verteilungen von Lösungsenergien zu bestimmen bzw. um Informationen auf die chemischen und strukturellen Eigenschaften der Zwischengitterplätze zu erhalten. Im amorphen System PdSi konnte so 1981 gezeigt werden [Kir88], dass es eine Gaußverteilung der Lösungsenergien der Zwischengitterplätze gibt, die direkt mit der Struktur der amorphen Proben korreliert ist. 1987 und 1989 zeigten [Har87] und [Kie89], dass die Lösungsenergien für Wasserstoff in binären NiZr- und NiTi-Legierungen als eine Superposition einzelner Gaußverteilungen betrachtet werden kann. Jede einzelne Verteilung entsprach dabei einem durch seine chemische Zusammensetzung bestimmten Zwischengitterplatztyp. Es konnte ebenfalls gezeigt werden, dass bei der Bestimmung der mittleren Energien sowohl chemische Einflüsse, als auch aus den Platzgrößen resultierende elastische Energiebeiträge berücksichtigt werden müssen.

Es war das Ziel der vorliegenden Arbeit diese Erkenntnisse und Modellierungen zu erweitern und auf Legierungssysteme anzuwenden, die aus mehr als nur zwei Komponenten bestehen. Die dazu verwendeten Legierungen waren zum einen die als Vitreloy bekannten Zr-Ni-Ti-Cu-Be-Legierungen [Pek93, Gey96]. Zum anderen wurde eine Zr-Al-Ni-CuLegierung [Zha91] (in dieser Arbeit ZANC abgekürzt) untersucht.

Diese Legierungen sind von besonderen Interesse, da sie als glasbildende Systeme, mit geringen Abschreckraten leicht herzustellen sind [Ino90, Zha91, Ino92, Pek93]. Da diese Materialien oft sehr spröde sind, gibt es große Bemühungen ihre mechanischen Eigenschaften $\mathrm{zu}$ verstehen. Eine besondere Fragestellung ist dabei der Einfluss der Konzentration des in einer solchen Legierungen gelösten Wasserstoffes auf die Härte oder die Rissbildung [Suh01]. In [Suh99] wurde zum Beispiel gezeigt, dass mit zunehmender Wasserstoffkonzentration ein Anstieg der Vickershärte in Zr-Ni-Ti-Cu-Be-Legierungen zu beobachten ist.

Neben den mechanischen Eigenschaften sind diese Gläser hinsichtlich ihrer Kristallisation sehr interessant. Führt man zum Beispiel mit entsprechenden Aufheizraten Kristallisationsexperimente durch, so sind in diesen Systeme quasikristalline 
Zwischenphasen mit einer fünffachen Symmetrie zu beobachten [Mac02]. Auch hier hat gelöster Wasserstoff einen entscheidenden Einfluss auf das Verhalten der Legierung [Ism00, Zan00].

Die genannten Legierungen wurden aus zwei weiteren Gründen ausgewählt. Sie unterscheiden sich zunächst in der Anzahl ihrer Komponenten (4 bzw. 5). Die Gültigkeit der Erweiterung der Modellierung des Wasserstofflösungsverhalten auf mehrkomponentige Systeme kann an ihnen geprüft werden. Des weiteren sind Unterschiede des Einflusses elastischer Energiebeiträge zu erwarten. Diese hängen von der Größe der vom Wasserstoff besetzten Plätze ab [Bro89a]. Die Platzgrößen wiederum korrelieren mit den Atomradien der Atome, die diese Plätze bilden. Während in der ZANC-Legeirung die Atomradien der beteiligten Elemente vergleichbar sind, enthalten die Vitreloy-Legierungen mit dem Be einen sehr kleinen Legierungspartner. Diese sehr kleinen Atome sollten zu einem merklichen Einfluss auf die Lösungsenergie führen.

Löst sich Wasserstoff inhomogen in diesen Legierungen, so treten in den Proben nicht zu vernachlässigende Spannungen auf [Dor02], die aufgrund der Sprödigkeit der Materialien zu einer Zerstörung der Probe führen können. Daher wurden in dieser Arbeit dünne Schichten dieser Legierungen untersucht. Diese Schichten wurden auf feste Substrate deponiert. Die Haftung auf diesen Substraten sorgte dafür, dass die dünnen Schichten während den Messungen stabil blieben.

Zur Bestimmung der Lösungsenergieverteilung wurden elektrolytisch Isothermen an den Legierungen gemessen. Darüber hinaus wurde an der $\mathrm{Zr}-\mathrm{Al}-\mathrm{Ni}-\mathrm{Cu}-\mathrm{Legierung}$ der intrinsische Diffusionskoeffizient des Wasserstoffes bestimmt. Dazu wurde eigens ein neues Verfahren entwickelt, dass es ermöglicht, quantitative Wasserstoff-Diffusionsexperimente an den Legierungen durchzuführen. Der Einsatz der herkömmlich Doppelzellenmessung kam aufgrund der Sprödigkeit und Zerstörung der Proben bei Wasserstoffaufnahme nicht in Frage.

In Kapitel 2 wird nach Vorstellung der Modellierung der Lösungsenergien für zweikomponentige Systeme die Erweiterung dieses Models auf mehrkomponentige Legierungen eingeführt. Die Probenherstellung, sowie die experimentellen Methoden, die in 
dieser Arbeit verwendet worden sind, sind im Kapitel 3 beschrieben. Die Präsentation der Messergebnisse erfolgt in Kapitel 4, bevor sie im Kapitel 5 diskutiert werden. Die Zusammenfassung der Arbeit stellt Kapitel 6 dar. 


\section{Wasserstoff in metallischen amorphen Legierungen}

\subsection{Berechnung von Platzdichteverteilungen im Sinne von Zustandsdichten für Wasserstoff in amorphen Legierungen}

In diesem Kapitel soll zunächst eine Einführung gegeben werden, auf welche Art in der Vergangenheit Platzdichteverteilungen für den Wasserstoff in binären amorphen Legierungen berechnet worden sind. Diese Platzdichteverteilungen beziehen sich auf die Häufigkeit bestimmter Tetraederplätze innerhalb eines Energieintervalls. Die Ähnlichkeit der Betrachtung lässt einen Vergleich mit den Zustandsdichten für Elektronen in einem Festkörper zu. Es werden statistische Überlegungen zur Häufigkeit der Tetraederplätze, die der Wasserstoff besetzen kann, angestellt. Die Energie eines solchen Platzes wird als Superposition der Lösungsenergien der einzelnen den Platz bildenden Elemente betrachtet. Später wird ein erweitertes Modell vorgestellt, dass es ermöglicht diesen Ansatz auch für Legierungen mit mehr als zwei Komponenten anzuwenden. Es wird bei der Berechnung der Platzenergien berücksichtigt, dass neben der Chemie auch elastische Energiebeiträge die Lösungsenergien für Wasserstoffatom beeinflussen.

\subsubsection{Zustandsdichten für den Wasserstoff in Kristallen und binären Legierungen}

Wasserstoff löst sich interstitiell in einem Metall und besetzt dabei atomare Zwischengitterplätze innerhalb des Kristallgitters. Die potentielle Energie, die ein Wasserstoffatom auf einem solchen Platz besitzt, hängt entscheidend von der chemischen nächsten Nachbarschaft und der Größe des Platzes ab. Man kann die Anzahl der Zwischengitterplätze innerhalb eines Energieintervalls in Analogie zu den Elektronenzuständen im Metall mit Hilfe einer Zustandsdichte Z(E) beschreiben. Die 
Zustandsdichte $\mathrm{Z}(\mathrm{E})^{1}$ gibt dabei an, welcher Bruchteil dN aller N Zwischengitterplätze eine Lösungsenergie für den Wasserstoff im Intervall [E, E+dE] besitzt. Wenn die Gesamtzahl der in einer Probe befindlichen Zwischengitterplätze $\mathrm{N}_{0}$ ist und jeder Platz die gleiche Lösungsenergie $\mathrm{E}_{0}$ für den Wasserstoff besitzt, so wird $\mathrm{Z}(\mathrm{E}) \mathrm{zu}$ einer Deltafunktion [Kir88]:

$$
Z(E)=\frac{d N}{d E}=N_{0} \delta\left(E-E^{0}\right)
$$

Die Fermienergie aus der Betrachtung der Elektronenzustände wird durch das chemische Potential des Wasserstoffes ersetzt, da bei $\mathrm{T}=0 \mathrm{~K}$ keine Plätze besetzt werden können, deren Energie größer als das chemische Potential des Wasserstoffes ist. Da jeder Zwischengitterplatz nur ein einziges Wasserstoffatom aufnehmen kann, wird die thermische Besetzung der Plätze durch den Wasserstoff mit Hilfe der Fermi-Dirac-Statistik beschrieben. Die thermische Besetzungswahrscheinlichkeit ist dann $1 /\left[1+\exp \left(\left(\mathrm{E}-\mu_{\mathrm{H}}\right) / \mathrm{RT}\right)\right]$, wobei $\mu_{\mathrm{H}}$ die Fermienergie, bzw. das chemische Potential des Wasserstoffes darstellt. Diese Funktion zeigt den bekannten Stufencharakter, d.h. sie ist 0 für $\mathrm{E}>\mu_{\mathrm{H}}$ und 1 für $\mathrm{E}<\mu_{\mathrm{H}}$. Die Summation über das Produkt aus Zustandsdichte $Z(E)$ und thermischer Besetzungswahrscheinlichkeit ergibt die Konzentration des Wasserstoffes [Kir88]:

$$
c_{H}=\int_{-\infty}^{+\infty} \frac{Z(E)}{1+\exp \left(\left(E-\mu_{H}\right) / R T\right)} d E
$$

In amorphen Materialien wurde gezeigt, dass sich der Wasserstoff quasi „interstitiell“ auf Tetraederplätzen löst [Sam83]. Diese Tetraederplätze sind durch die amorphe Struktur in ihrer Größe und Form und somit auch in ihrer Energie von einander unterschiedlich. Daraus ergibt sich für $\mathrm{Z}(\mathrm{E})$ eine Platzenergieverteilung statt einer Delta-Funktion. Bereits die Anzahl aller in einem Material vorkommender Tetraeder eines einzelnen Typs ist so groß, dass eine diskrete Beschreibung der Platzenergieverteilung durch eine kontinuierliche ersetzt werden kann.

\footnotetext{
${ }^{1}$ Streng genommen handelt es sich bei Z(E) um eine Zwischengitterplatzhäufigkeitsverteilung, die in dieser Arbeit als Zustandsdichte benannt wird.
} 
Wenn die Breite der Verteilung $\mathrm{Z}(\mathrm{E})$ in Gleichung (2) viel größer als RT ist, und das chemische Potential innerhalb der Verteilung $Z(E)$ liegt, kann die bereits erwähnte Stufennäherung auf diese Gleichung angewendet werden. Das bedeutet, dass in erster Näherung nur Plätze in der Verteilung $Z(E)$ besetzt werden können, die energetisch unterhalb des chemischen Potentials $\mu_{\mathrm{H}}$ liegen. In diesem Fall reicht es aus, die Integration nur bis $\mu_{\mathrm{H}}$ statt bis $+\infty$ durchzuführen. Damit vereinfacht sich Gleichung (2) zu :

$$
c_{H}=\int_{-\infty}^{\mu_{H}} Z(E) d E
$$

Differenzieren von Gleichung (3) unter Anwendung der Leibnitzschen Regel [Kim88] führt $\mathrm{zu}$

$$
\frac{d c_{H}}{d \mu_{H}}=Z(\mu)
$$

Mit Gleichung (4) kann bei Kenntnis des Verlaufs einer Isotherme in Form von $\mu_{\mathrm{H}}\left(\mathrm{c}_{\mathrm{H}}\right)$ die entsprechende Zustandsdichte bestimmt werden. (s. auch Kapitel 3.2). Da die Verteilung der NN - Abstände in einem amorphen Festkörper, d. h. das erste Maximum in der radialen Verteilungsfunktion (RDF), durch Gaußfunktion beschrieben werden kann, kann man annehmen, dass auch die platzgrößenabhängige Zustandsdichte Z(E) eine Gauß - Verteilung zeigt. Aus der Deltafunktion in der Zustandsdichte in Gleichung (1) wird dann [Kir82, Kir83]:

$$
Z(E)=\frac{1}{\sigma \sqrt{\pi}} \exp \left[-\left(\frac{E-E^{0}}{\sigma}\right)^{2}\right]
$$

Für die Halbwertsbreite $\sigma$ wurden in amorphen Legierungen, wie PdSi [Kir82] und NiZr [Kie89] Werte in der Nähe von $\sigma=10 \mathrm{~kJ} / \mathrm{mol}$ gefunden. Die Energie $\mathrm{E}^{0}$ in Gleichung (5) stellt die mittlere Energie der Verteilung dar und wird am stärksten durch die chemische Zusammensetzung der vier Atome bestimmt, die den Tetraeder bilden, also den nächsten Nachbarn eines gelösten Wasserstoffatoms. 
Löst sich Wasserstoff in einem Festkörper, so werden zuerst die Plätze mit möglichst niedrigen Energien besetzt [Kir88]. Zur Beschreibung des Wasserstofflösungsverhaltens reicht daher eine Betrachtung der Verteilung der Minima, wie in Abbildung 1 schematisch dargestellt, aus.
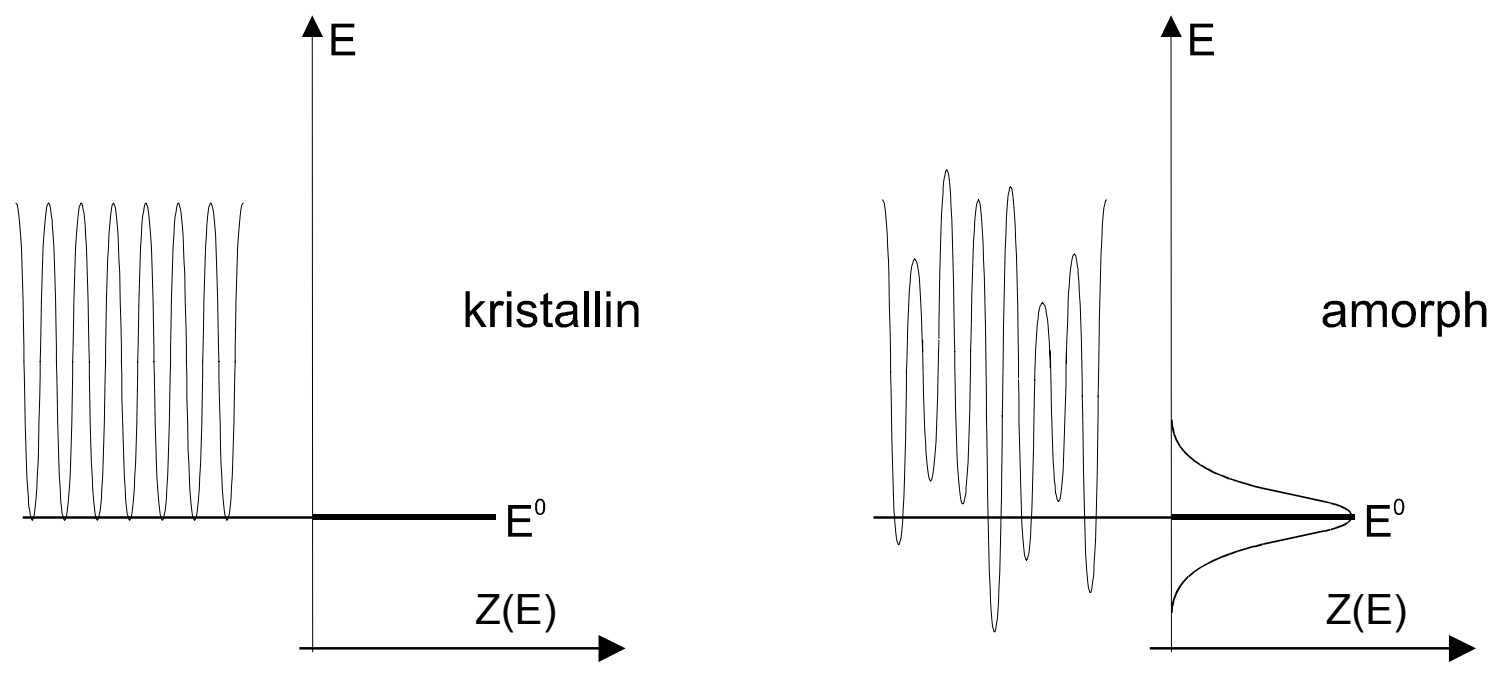

Abbildung 1: skizzierte Zustandsdichten Z(E) für einen kristallinen und einen amorphen Festkörper, die jeweils nur aus einer Atomsorte bestehen.

Die bisher angestellten Überlegungen bezogen sich lediglich auf einkomponentige Systeme. In Legierungen, die aus zwei metallischen Komponenten, A und B, bestehen, sind die fünf verschiedene Tetraedertypen in Abbildung 2 denkbar:
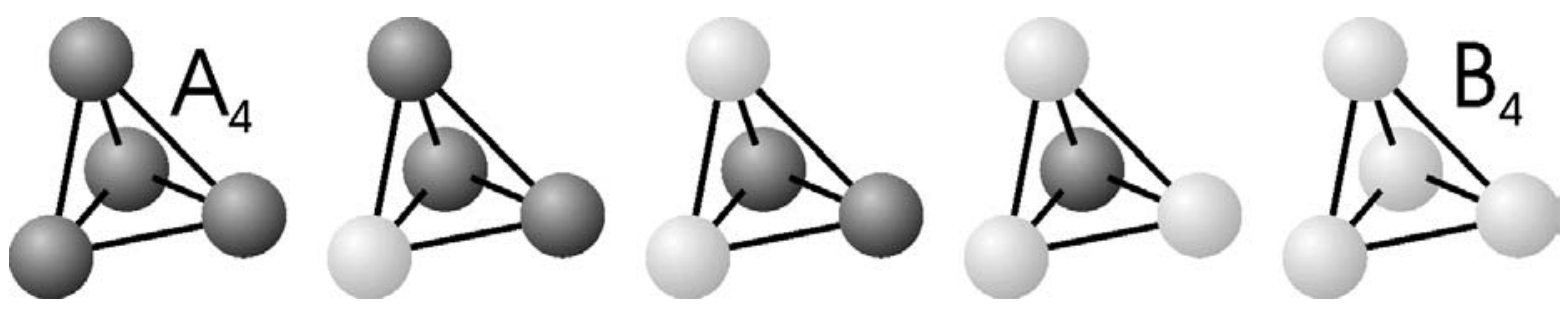

Abbildung 2: In einer zweikomponentigen A-B-Legierung ist die Konstruktion von fünf chemisch voneinander verschiedenen Tetraedern möglich: $A_{4}, A_{3} B_{1}, A_{2} B_{2}, A_{1} B_{3}$ und $B_{4}$. 
Jeder Zwischengitterplatz der Tetraeder $A_{4}, A_{3} B_{1}, A_{2} B_{2}, A_{1} B_{3}$ und $B_{4}$ besitzt aufgrund der fünf verschiedenen chemischen Nachbarschaften für den Wasserstoff eine andere mittlere Lösungsenergie $\mathrm{E}_{\mathrm{i}}^{0}$. Die genauen mittleren Energien eines jeden Tetraeders wird später ausführlich diskutiert. Eine erste Abschätzung der Platzenergien ist jedoch mit Gleichung (6) möglich [Har87]:

$$
E_{i}^{0}=\frac{\left(a \cdot E_{A}^{0}+b \cdot E_{B}^{0}\right)}{a+b}
$$

Die Zahlen a und $\mathrm{b}$ geben an, wie oft das Atom A bzw. das Atom B in einem Tetraeder $\mathrm{i}$ enthalten sind. Die Energie eines Tetraederplatzes berechnet sich hiernach als dass Mittel der Lösungsenergien $\mathrm{E}_{\mathrm{A}}^{0}$ und $\mathrm{E}_{\mathrm{B}}{ }^{0}$ des Wasserstoffes auf den reinen Tetraederplätzen $\mathrm{A}_{4}$ und $\mathrm{B}_{4}$.

$\mathrm{Z}(\mathrm{E})$ kann in einer binären amorphen $\mathrm{A}-\mathrm{B}$ - Legierung durch eine Überlagerung von fünf Normalverteilungen (eine für jeden Tetraederplatz i), beschrieben werden [Kie89].

$$
Z(E)=\frac{\sum_{i=1}^{5} n_{i} \frac{1}{\sigma_{i} \sqrt{\pi}} \exp \left[-\left(\frac{E-E_{i}^{0}}{\sigma_{i}}\right)^{2}\right]}{\sum_{i=1}^{5} n_{i}}
$$

wobei $\mathrm{n}_{\mathrm{i}}$ die relative Häufigkeit ist, mit der ein Tetraedertyp in der Legierung vorkommt. Diese Häufigkeit hängt nach Gleichung (8) von der Konzentration x der Komponente B in der Legierung ab. Der Parameter b entspricht der Anzahl der B-Atome auf den Ecken des Tetraeders des Typs i:

$$
n_{i}=\frac{\text { Tetraeder vom Typ i }}{\text { alle Tetraederim Material }}=\left(\begin{array}{l}
4 \\
b
\end{array}\right) x^{b}(1-x)^{4-b}
$$

Die Häufigkeiten, die sich für die fünf verschieden möglichen Tetraeder in einer $\mathrm{A}_{0,75} \mathrm{~B}_{0,25}-$ Legierung ergeben, sind in der Abbildung 3 zu sehen. 


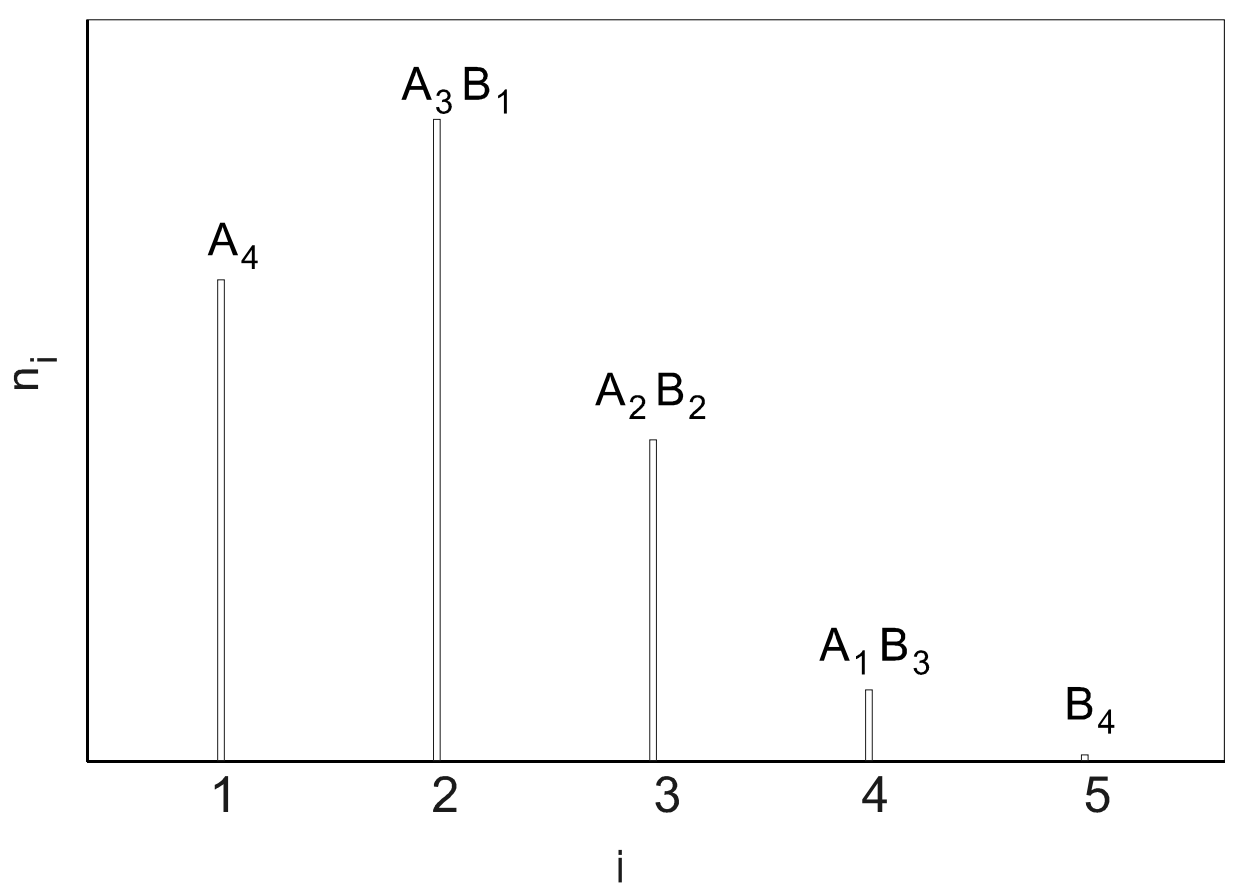

Abbildung 3: relative Häufigkeit $n_{i}$ der Tetraeder $i$ in der Beispiellegierung $A_{0.75} B_{0.25}$

\subsubsection{Berechnung von Isothermen}

Es ist darauf $\mathrm{zu}$ achten, dass $\mathrm{Z}(\mathrm{E})$ eine Platzdichteverteilung darstellt. Somit führt die Berechnung der Gleichung (2) zu einer "Wasserstoff pro Tetraederplatz" - Konzentration. Üblicherweise wird die Wasserstoffkonzentration aber als $\mathrm{H} / \mathrm{Me}$ also "Wasserstoff pro Metallatom" angegeben. Zur Umrechnung dieser Konzentrationen benötigt man einen Umrechnungsfaktor f, der Zahl an Tetraedern entspricht, die im Mittel von einem Atom gebildet werden. Für die Konzentrationen, also Wasserstoffatome pro Metallatome $\mathrm{c}_{\mathrm{H}, \text { At }}$ und Wasserstoff pro Tetraederplätze $\mathrm{c}_{\mathrm{H} \text {, Tet }}$ folgt somit:

$$
c_{H, A t}=f \cdot c_{H, T e t}
$$

bzw. für die entsprechenden Zustandsdichten:

$$
Z_{H, A t}(E)=f \cdot Z_{H, T e t}(E)
$$


Im dichtest gepackten fcc-Gitter, in dem 4 Atome in einer Elementarzelle zwölf Zwischengitterplätze bilden, gilt $\mathrm{f}=3$. Für den hypothetischen Fall, ein Festkörper wäre komplett aus Tetraedern mit gleichseitigen Dreiecksflächen zusammengesetzt, ist eine Abschätzung zu f $=5$ denkbar: Das mittlere Atom in einem Ikosaeder ist jeweils zu einem Teil in einem der 20 den Ikosaeder bildenden Tetraeder. Rein rechnerisch besteht dann der Tetraeder aus 4 Atomen, die zu einem zwanzigstel $(1 / 20=0,05)$ in ihm enthalten sind, also aus $0,2=1 / 5$ Atomen, womit $f=5$ wäre. Einen solchen Festkörper gibt es jedoch nicht. Der für eine amorphe Struktur zu erwartende Wert sollte in dem Intervall $f=3$ und $f=5$ liegen. In dieser Arbeit werden auch im späterem Verlauf nur die Platzdichtenverteilungen, also $\mathrm{Z}_{\mathrm{H}, \mathrm{Tet}}(\mathrm{E})$ in Gleichung (10), diskutiert und in der Form $\mathrm{Z}(\mathrm{E})$ benannt. Experimentell ermittelte Zustandsdichten werden entsprechend umgerechnet.

Zur Berechnung von $\mu_{\mathrm{H}}\left(\mathrm{c}_{\mathrm{H}}\right)$ aus modellierten Zustandsdichten $\mathrm{Z}(\mathrm{E})$ müsste man die Gleichung (2) nach $\mu_{\mathrm{H}}$ auflösen. Analytisch ist dies nur für den Fall möglich, dass es sich bei Z(E) um eine einzige Gaußverteilung handelt. Für Überlagerungen von Gaußkurven ist diese Auflösung nicht mehr geschlossen durchführbar. Eine Bestimmung von $\mu_{\mathrm{H}}\left(\mathrm{c}_{\mathrm{H}}\right)$ ist für kompliziertere Z(E) nur möglich, in dem die Integration in Gleichung (2) numerisch durchgeführt wird und der gewonnenen $\mathrm{c}_{\mathrm{H}}\left(\mu_{\mathrm{H}}\right)$-Verlauf in der achsenvertauschten Form $\mu_{\mathrm{H}}\left(\mathrm{c}_{\mathrm{H}}\right)$ betrachtet wird. 


\subsection{Diffusion von $\mathrm{H}$ in amorphen Legierungen}

Im folgenden Abschnitt wird eine kurze Herleitung präsentiert, mit der die Druckabhängigkeit des Wasserstoffdiffusionskoeffizienten in einer amorphen Umgebung berechnet werden kann. Zunächst gilt für den Tracerdiffusionskoeffizienten [Kir85]:

$$
D^{*}=D^{0} \frac{\left(1-c^{2}\right)}{c} \exp \left(\frac{\mu_{H}-G^{0}}{R T}\right)
$$

$\mathrm{D}^{*}$ beschreibt die Diffusion von Teilchen in einem Gitter ohne vorhandenen makroskopischen Konzentrationsgradienten. Der Parameter $\mathrm{D}^{0}$ hat die Bedeutung eines Diffusionskoeffizienten in einem hypothetischen Referenzmaterial, in denen alle dem diffundierenden Teilchen zur Verfügung stehenden Plätze die gleiche Energie $G^{0}$ besitzen und die zu überwindende Energiebarriere für einen Platzwechsel einer festen Energie $\mathrm{Q}^{0}$, der Aktivierungsenergie, entspricht. In dieser Arbeit wird $\mathrm{D}^{0}$ als Fitparameter benutzt. Der Vorfaktor $(1-\mathrm{c})^{2} / \mathrm{c}$ beschreibt die Abnahme freier Plätze mit zunehmender Wasserstoffkonzentration und die Änderung der thermodynamischen Aktivität mit zunehmender Füllung des Gitters. Der Ausdruck $\left.\exp \left[\left(\mu_{\mathrm{H}^{-}} \mathrm{G}^{0}\right) / \mathrm{RT}\right)\right]$ sagt aus, dass hauptsächlich der Wasserstoff nahe dem chemischen Potential zur Diffusion beiträgt.

Mit der Definition der Aktivität $\lambda^{0}=\exp \left[\left(\mu_{\mathrm{H}^{-}} \mathrm{G}^{0}\right) / \mathrm{RT}\right]$ und dem Aktivitätskoeffizienten $\gamma^{0}=\lambda^{0} / \mathrm{c}_{\mathrm{H}}$ ergibt sich für $\mathrm{D}^{*}$ :

$$
D^{*}=D^{0} \cdot \gamma^{0}
$$

Dabei wurde die Annahme gemacht, dass die Konzentration c sehr klein gegen 1 ist, so das der Term (1-c $\left.{ }^{2}\right)$ in Gleichung (11) vernachlässigt werden kann. Für den intrinsischen Wasserstoffdiffusionskoeffizienten $D_{i n}$ in einer amorphen Legierung gilt [Kir85]:

$$
D_{i n}=D^{*} \cdot\left(1+\frac{\delta \ln \gamma^{0}}{\delta \ln c_{H}}\right)
$$


Der Klammerterm in Gleichung (13) stellt den sogenannten thermodynamischen Faktor dar und berücksichtigt thermodynamische Triebkräfte bei der Diffusion. Führt man die Differentiation nach Einsetzen von $\gamma^{0}$ in Gleichung (13) durch, so folgt mit Gleichung (12) für den intrinsischen Diffusionskoeffizienten bei der Wasserstoffkonzentration $\mathrm{c}_{\mathrm{H}}$ :

$$
D_{i n}\left(c_{H}^{*}\right)=\left.D^{0} \cdot \gamma^{0} \cdot \frac{c}{R T} \cdot \frac{\delta \mu_{H}}{\delta c_{H}}\right|_{c_{H}^{*}}=\left.D^{0} \cdot \lambda^{0} \cdot \frac{1}{R T} \cdot \frac{\delta \mu_{H}}{\delta c_{H}}\right|_{c_{H}^{*}}
$$

Besitzt man Kenntnis über die Isotherme $\mu_{\mathrm{H}}\left(\mathrm{c}_{\mathrm{H}}\right)$ so kann der Term $\delta \mu_{\mathrm{H}} / \delta \mathrm{c}_{\mathrm{H}}$ an der Stelle $\mathrm{c}_{\mathrm{H}}=\mathrm{c}_{\mathrm{H}}^{*}$ in Gleichung (14) und damit auch der konzentrationsabhängige Diffusionskoeffizient $\mathrm{D}_{\text {in }}\left(\mathrm{c}^{*}{ }_{\mathrm{H}}\right)$ bestimmt werden:

$$
D_{i n}\left(c_{H}\right)=\left.\frac{D^{0}}{R T} \cdot \frac{\delta \mu_{H}}{\delta c_{H}}\right|_{c_{H}^{*}} \cdot \sqrt{\frac{p_{H 2}}{p_{H 2,0}}}
$$

in Gleichung (15) wurde benutzt, dass das chemische Potential $\mu_{\mathrm{H}}$ bei einem Wasserstoffdruck $\mathrm{p}_{\mathrm{H} 2}$ gleich $\mu_{\mathrm{H}}=\mu_{\mathrm{H}, 0}+(\mathrm{RT} / 2) \ln \left[\mathrm{p}_{\mathrm{H} 2} / \mathrm{p}_{\mathrm{H} 2,0}\right]$ und die Aktivität somit $\lambda^{0}=\left(\mathrm{p}_{\mathrm{H} 2} / \mathrm{p}_{\mathrm{H} 2,0}\right)^{1 / 2}$ ist. Für eine feste Temperatur und eine bestimmte Wasserstoffkonzentration $\mathrm{c}_{\mathrm{H}}^{*}$ in der Probe ist also der intrinsische Diffusionskoeffizient proportional zur Wurzel des angelegten Wasserstoffdruckes. 


\subsection{Zustandsdichten mehrkomponentiger amorpher Legierungen}

Auf den Grundlagen des Kapitels 2.1 wurde in dieser Arbeit ein Modell entwickelt, mit dessen Hilfe sich die Zustandsdichten Z(E) auch für amorphe Legierungen, die aus mehr als nur aus zwei Elementen bestehen, berechnen lassen. Eine solche Zustandsdichte lässt sich ebenfalls als Überlagerung mehrerer Gaußverteilungen darstellen. Jede einzelne Gaußverteilung stellt die Energieverteilung eines einzelnen Tetraederplatztyps i um den mittleren Wert $\mathrm{E}_{\mathrm{i}}^{0}$ dar. $\mathrm{Zu}$ Beginn dieses Kapitels werden zunächst statistische Überlegungen angestellt, um zu berechnen, wie viele verschiedene Tetraeder mit welcher Wahrscheinlichkeit in einer beliebigen Legierung vorkommen. Anschließend wird ein Modell zur Berechnung der mittleren Energien $\mathrm{E}_{\mathrm{i}}^{0}$ der jeweiligen Tetraederplätze unter Berücksichtigung von sowohl chemischen als auch elastischen Energiebeiträgen entwickelt. Schließlich wird eine neue Betrachtung der Wasserstoff - Wasserstoff - Wechselwirkung, wie sie in dieser Arbeit Verwendung findet, vorgestellt.

\subsubsection{Häufigkeitsverteilung von Tetraederplätzen in einer homogenen amorphen Legierung}

Um die Gleichung (7) nun auf eine beliebige m-komponentige Legierung zu erweitern, muss sowohl die Anzahl aller möglichen Tetraeder als auch die Wahrscheinlichkeit, mit der ein bestimmter Tetraeder in der Legierung vorliegt, bestimmt werden.

Da in die Platzenergien nur die chemische Umgebung und die Größe jedes Tetraederplatzes eingehen, ist es irrelevant, welche der vier Tetraederecken bei gegebener Zusammensetzung des Tetraeders durch ein herausgegriffenes Atom besetzt werden. Danach ergibt sich die Anzahl von Tetraedern unterschiedlicher Zusammensetzung als Anzahl der Kombinationen mit Wiederholung, d.h. die Anordnung der Atome im Tetraeder ist irrelevant und eine einzelne der m Legierungskomponenten kann keinmal, einmal oder bis zu viermal im Tetraeder auftreten. Daher gilt [Bro77]: 


$$
K=\left(\begin{array}{c}
m+k-1 \\
k
\end{array}\right)=\frac{(m+3) !}{4 !(m-1) !}
$$

für den Fall einer $\mathrm{m}$ - komponentigen Legierung aus der Tetraeder mit $\mathrm{k}=4$ Atomen gebildet werden. Tabelle 1 zeigt das Ergebnis der Gleichung (16) als Funktion von m für die Bildung von Tetraedern $(\mathrm{k}=4)$.

Tabelle 1: Mit zunehmender Anzahl der Legierungskomponenten m steigt die Zahl der verschiedenen Tetraedertypen K, die gebildet werden können.

\begin{tabular}{c|cccccc}
\hline $\mathrm{m}$ & 1 & 2 & 3 & 4 & 5 & 6 \\
\hline \hline $\mathrm{K}$ & 1 & 5 & 15 & 35 & 70 & 126 \\
\hline
\end{tabular}

Wie man sieht, können in einer Legierung aus drei verschiedenen Atomsorten schon 15 verschiedene Tetraedertypen vorkommen. In einer fünfkomponentigen Legierung gibt es sogar bereits 70 verschiedene Plätze, die in Gleichung (7) zu berücksichtigen sind. Jeder Tetraeder (Index i) aus der Menge der $\mathrm{K}$ möglichen Tetraeder wird durch $\mathrm{m}$ Besetzungszahlen $n_{i j}$ beschrieben. Die Besetzungszahlen können dabei Werte von $n_{i j}=0$ (die Komponente $\mathrm{j}$ tritt in dem Tetraeder nicht auf) bis $\mathrm{n}_{\mathrm{ij}}=4$ (die Komponente bildet den Tetraeder ganz alleine) vorkommen. Ferner gilt (genau vier Atome bilden einen Tetraeder):

$$
\sum_{j=1}^{m} n_{i j}=4
$$

Die relative Häufigkeit jedes Tetraedertyps hängt von der Anzahl der Realisierungsmöglichkeiten und der Zusammensetzung der Legierung ab. Für einen Tetraeder vom Typ $\mathrm{n}_{\mathrm{ik}}=4, \mathrm{n}_{\mathrm{ij}}=0 \forall \mathrm{j} \neq \mathrm{k}$ gibt es genau eine Realisierungsmöglichkeit: alle vier Ecken sind mit Atomen der Komponente $\mathrm{k}$ besetzt. Für einen Tetraeder des Typs $\mathrm{n}_{\mathrm{ik}}=3, \mathrm{n}_{\mathrm{il}}=1, \mathrm{n}_{\mathrm{ij}}=0 \forall$ $\mathrm{j} \neq \mathrm{k} \wedge \mathrm{j} \neq 1$ gibt es genau vier Realisierungsmöglichkeiten: Das Atom der Komponente 1 kann jede der vier Ecken des Tetraeders besetzen. Die Anzahl der Realisierungsmöglichkeiten ergibt sich also als Anzahl der Permutationen mit Wiederholung (Anordnung ist relevant, eine Komponente kann keinmal oder bis zu viermal auftreten) [Bro77]: 
(18)

$$
P_{i}=\frac{4 !}{\prod_{j=1}^{m} n_{i j} !}
$$

Liegt eine Legierung aus $m$ Elementen mit den Konzentrationen $x_{j}$ der jeweiligen Komponente $\mathrm{j}$ vor, so ist die relative Häufigkeit eines Tetraeders i gegeben durch:

$$
X_{i}=\prod_{j=1}^{m} x_{j}^{n_{i j}}
$$

Die Wahrscheinlichkeit, dass ein Tetraedertyp $\mathrm{i}$ in der Legierung auftritt, berechnet sich aus dem Produkt von $\mathrm{P}_{\mathrm{i}}$ und $\mathrm{X}_{\mathrm{i}}$.

$$
W_{i}=P_{i} \cdot X_{i}
$$

In Gleichung (7) muss nun die Summation durch eine Summation über K und die $n_{i}$ durch $\mathrm{W}_{\mathrm{i}}$ aus Gleichung (20) ersetzt werden.

Der Formelapparat der Gleichungen (16) bis (20) wurde zur Veranschaulichung an der binären Beispiellegierung $\mathrm{A}_{0.75} \mathrm{~B}_{0.25}(\mathrm{~m}=2 \rightarrow \mathrm{K}=5)$ exemplarisch durchgerechnet und in Tabelle 2 zusammengefasst.

Tabelle 2: Tetraeder in einer Beispiellegierung $A_{0.75} B_{0.25}$

\begin{tabular}{|c|c|c|c|c|c|}
\hline Tetraeder: & $\mathrm{A}_{4}$ & $\mathrm{~A}_{3} \mathrm{~B}_{1}$ & $\mathrm{~A}_{2} \mathrm{~B}_{2}$ & $\mathrm{~A}_{1} \mathrm{~B}_{3}$ & $\mathrm{~B}_{4}$ \\
\hline \hline $\mathbf{I}$ & 1 & 2 & 3 & 4 & 5 \\
$\underline{\mathbf{T}}_{\mathbf{i}}(\mathbf{a}, \mathbf{b})$ & $(4,0)$ & $(3,1)$ & $(2,2)$ & $(1,3)$ & $(0,4)$ \\
$\mathbf{P}_{\mathbf{i}}$ & 1 & 4 & 6 & 4 & 1 \\
$\mathbf{W}_{\mathbf{i}}$ & 0,3164 & 0,1055 & 0,0352 & 0,0117 & 0,0039 \\
$\mathbf{n}_{\mathbf{i}}$ & $\mathbf{0 , 3 1 6 4}$ & $\mathbf{0 , 4 2 2 0}$ & $\mathbf{0 , 2 1 1 2}$ & $\mathbf{0 , 0 4 6 8}$ & $\mathbf{0 , 0 0 3 9}$ \\
\hline
\end{tabular}

Wie zu erwarten war, ergaben sich hier für die $n_{i}$ die bereits in Abbildung 3 gezeigte Häufigkeitsverteilung. 


\subsubsection{Bestimmung der mittleren Energien der einzelnen Gaußverteilungen}

Die Energien $\mathrm{E}_{\mathrm{A}}{ }^{0}$ und $\mathrm{E}_{\mathrm{B}}{ }^{0}$ in der Gleichung (6), die eine erste Abschätzung der Lösungsenergien [Har87] ergab, stellten die Lösungsenergien von Wasserstoff auf den Tetraederplätzen $\mathrm{A}_{4}$ und $\mathrm{B}_{4}$ in den reinen Elementen $\mathrm{A}$ und $\mathrm{B}$ dar. Diese Energiebeiträge kommen zum einen durch die chemische Nachbarschaft zustande. Zum anderen muss aber beachtet werden, dass in jedem Fall eine strukturelle Aufweitung eines Tetraederplatzes durch die Besetzung mit einem Wasserstoffatom bedingt ist. Die Energien $\mathrm{E}_{\mathrm{A}}{ }^{0}$ und $\mathrm{E}_{\mathrm{B}}{ }^{0}$ enthalten diese elastischen Energiebeiträge bereits für die Tetraeder in den reinen Elementen. Wenn ein A4-Tetraeder in einer A-B-Legierung ein anderes Volumen besitzt, als in dem reinen Element A, so muss eine Veränderung des Tetraederplatzvolumens bei der Platzenergieberechnung durch zusätzliche elastische Energiebeiträge berücksichtigt werden. In [Bro89a] konnte an NbV-Legierungen gezeigt werden, dass die mittleren Energien der Tetraederplätze stark von den Konzentrationen des Nb bzw. V abhängen und auf eine Volumenveränderung der Plätze im Vergleich zu freien Tetraedern zurückzuführen sind. Die Berücksichtigung der Tetraederplatzgrößen geschieht im Rahmen der von [Bro89a] angewendeten Volumenabschätzung des "eingebetteten clusters". Unter der Annahme, dass der Wasserstoff als Kugel einen kugelförmigen Tetraederplatz, der in einer homogenen isotropen Matrix eingebettet ist, besetzt, wird der resultierende Energiebeitrag schließlich nach Eshelby berechnet.

Das Platzvolumen $\mathrm{V}^{0}{ }_{\mathrm{i}}$ des freien Tetraeders i kann im Modell starrer Kugeln abgeschätzt werden. $\mathrm{V}^{0}{ }_{\mathrm{i}}$ wird im folgenden als das maximale Volumen einer Kugel, die noch auf einen solchen Tetraederplatz passt, behandelt. Dabei wird angenommen, dass das Volumen eines freien Tetraeders vom Typ $\mathrm{n}_{\mathrm{ik}}=4, \mathrm{n}_{\mathrm{ij}}=0 \forall \mathrm{j} \neq \mathrm{k}$, genauso groß ist, wie der Tetraederplatz in dem reinen Element k. Der Radius der diesem Tetraeder eingeschriebenen Kugel beträgt [Fuk93]( $\mathrm{R}_{\mathrm{k}}$ : Atomradius des Elements $\left.\mathrm{k}\right)$ :

$\begin{array}{ll}\text { fcc/hcp-Gitter: } & 0.22 \times \mathrm{R}_{\mathrm{k}} \\ \text { bcc-Gitter: } & 0.29 \times \mathrm{R}_{\mathrm{k}}\end{array}$ 
Im folgenden wird unter Berücksichtigung dieser beiden Beziehungen näherungsweise angenommen, dass in dem reinen Element $\mathrm{k}$, mit dem Atomradius $\mathrm{R}_{\mathrm{k}}$, für den maximalen Radius $\mathrm{R}_{\mathrm{k} 4}$ einer eingeschriebenen Kugel auf einem Tetraederplatz gilt:

$$
R_{k 4}=\frac{1}{4} R_{k}
$$

Dementsprechend errechnet sich das Volumen $\mathrm{V}^{0}{ }_{\mathrm{k} 4}$ dieser Kugel zu:

$$
V_{k 4}^{0}=\frac{4}{3} \pi \cdot\left(R_{k 4}^{0}\right)^{3} \cong \frac{1}{48} \pi \cdot R_{k}^{3}
$$

Besteht ein freier Tetraeder i aus vier Atomen mit unterschiedlichen Atomradien $R_{k}$, so wird das Lochvolumen des freien Tetraeders $\mathrm{V}^{0}{ }_{\mathrm{i}}$ als arithmetisches Mittel zwischen den Lochvolumina $\mathrm{V}_{\mathrm{k} 4}^{0}$ freier Tetraeder, die nur aus einer Atomsorte bestehen, abgeschätzt:

$$
V_{i}^{0}=\frac{1}{4} \sum_{k} V_{k 4}^{0}=\frac{\pi}{192} \sum_{k} R_{k}^{3}
$$

Nachdem das Volumen $\mathrm{V}^{0}{ }_{\mathrm{i}}$ eines freien Tetraeders in dem vorhergehenden Abschnitt berechnet worden ist, soll nun die Größe eines Tetraederplatzes in einer Legierung aus m Elementen abgeschätzt werden. Es wird zunächst die Annahme gemacht, dass alle sich in einer Legierung befindlichen Tetraederplätze das gleiche mittlere Volumen $<\mathrm{V}>$ besitzen. Im Modell starrer Kugeln folgt, dass dieses Volumen direkt proportional zu dem Molvolumen $\mathrm{V}_{\text {mol, Leg }}$ dieser Legierung sein muss:

$$
\langle V\rangle \propto V_{m o l, L e g}
$$

Für zwei verschiedene Legierungen $V_{\text {mol, Leg a }}$ und $V_{\text {mol, Leg } b}$ folgt aus dieser Proportionalität für das Verhältnis ihrer mittleren Volumina $\left\langle\mathrm{V}>^{\mathrm{a}} \text { und }<\mathrm{V}\right\rangle^{\mathrm{b}}$ : 


$$
\frac{\langle V\rangle^{a}}{\langle V\rangle^{b}}=\frac{V_{m o l, \operatorname{Leg} a}}{V_{m o l, \operatorname{Leg} b}}
$$

Das Molvolumen einer solchen Legierung wird mit Hilfe des Vegardschen Gesetzes berechnet:

$$
V_{\text {mol, Leg }}\left(x_{A}\right)=x_{A} \cdot V_{\text {mol,Element } A}+\left(1-x_{A}\right) \cdot V_{\text {mol,Element } B}
$$

bzw. bei mehr als zwei Legierungskomponenten:

$$
V_{m o l, L e g}\left(x_{i}\right)=\sum_{i=1}^{m} x_{i} \cdot V_{m o l, i}
$$

Wenn nun statt der Legierung B das reine Element k als Referenz in Gleichung (25) gesetzt wird, so folgt (das mittlere Volumen $\langle\mathrm{V}\rangle^{\mathrm{b}}$ beträgt dann $\langle\mathrm{V}\rangle^{\mathrm{b}}=\langle\mathrm{V}\rangle^{\mathrm{k}}=\mathrm{V}^{0}{ }_{\mathrm{k} 4}$ ):

$$
\langle V\rangle=V_{k 4}^{0} \frac{V_{m o l, L e g}}{V_{m o l, k}}
$$

Mit Hilfe dieses mittleren Volumens, dass sich für jede Legierung einfach berechnen lässt, folgt sofort durch Subtrahieren von Gleichung (23), die Volumenveränderung eines Tetraeders $i$ in der Legierung im Vergleich zu seinem freien Zustand:

$$
\Delta V_{i}=\frac{V_{m o l, L e g}}{V_{m o l, k}} V_{k 4}^{0}-V_{i}^{0}
$$

Die Abbildung 4 verdeutlicht diesen Zusammenhang noch einmal. Es ist sehr gut zu erkennen, dass in der A-B-Legierung mit $\mathrm{x}_{\mathrm{Leg}}$ die $\mathrm{A}_{4}$ - Tetraeder kleiner, die $\mathrm{B}_{4}-$ Tetraeder jedoch größere als in ihren reinen Elementen sind. $\Delta \mathrm{V}_{\mathrm{i}}$ (durch Pfeile skizziert) kann daher sowohl positiv als auch negativ sein. 


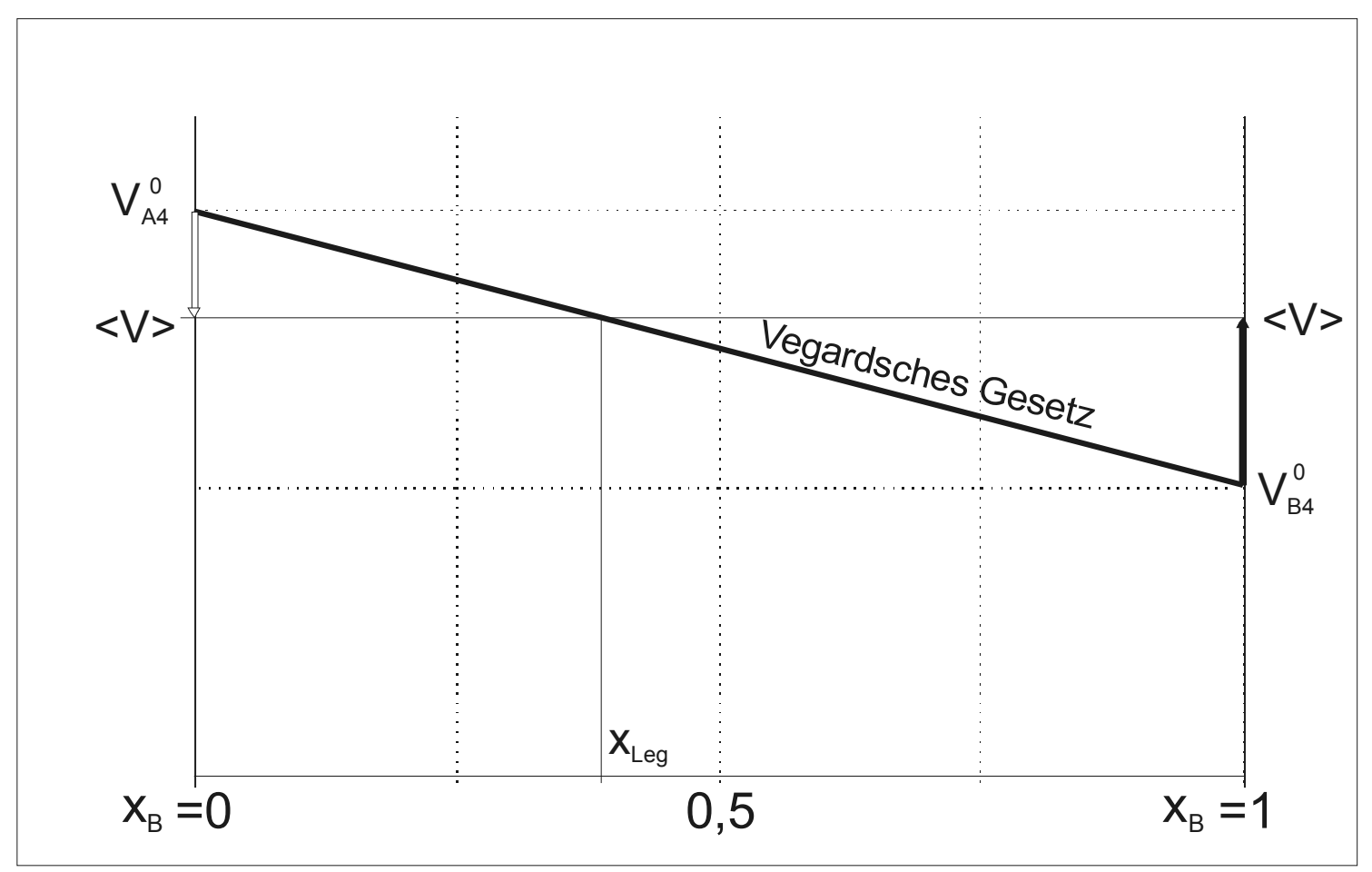

Abbildung 4: Mit Hilfe des Gesetzes von Vegard lässt sich mit Gleichung (28) das mittlere Tetraedervolumen $<V>$ aller Tetraeder in einer Legierung der Konzentration $x_{\text {Leg }}$ berechen. Im Vergleich zu ihren freien Äquivalenten können Tetraederplätze dadurch sowohl größer als auch kleiner werden. Hier ist dies für die A4 und B4 - Tetraeder skizziert.

Die bis hierhin angestellten Überlegungen berücksichtigen noch nicht die lokalen Tetraedergrößen in dem Gitter, d.h. die bisherige Annahme, das $<\mathrm{V}>$ das Volumen aller Tetraeder sei, wird fallen gelassen. Es ist zu erwarten, dass die Größe eines Tetraedertyps durchaus von der eines anderen Tetraedertyps unterschiedlich sein kann. Es wird nun die Annahme gemacht, dass das Tetraedervolumen $V_{i}$ eines Tetraeders $i$ in einer amorphen Legierung zwischen $\langle\mathrm{V}\rangle$ und dem Volumen eines freien Tetraeders $\mathrm{V}_{\mathrm{i}}^{0}$ liegt. Diese Annahme ist sinnvoll, da jeder Tetraeder das Bestreben hat, sich in seinen freien Zustand zu begeben. Der Einbau in eine Legierung hindert ihn daran. Diese Abschätzung wird durch einen Laufparameter S vorgenommen, der nur Werte zwischen 0 und 1 annehmen kann [Bro89a].

$$
V_{i}=S \cdot\langle V\rangle+(1-S) V_{i}^{0}
$$


Der Fall $\mathrm{S}=1$ bedeutet, dass alle amorphen Tetraederplätze in einer Legierung gleich groß sind. Alle haben das gleiche mittlere Volumen $<\mathrm{V}>$. Für $\mathrm{S}=0$ gilt, dass alle Tetraederplätze ein unterschiedliches Volumen haben. Dieses entspricht dann dem Volumen $\mathrm{V}_{\mathrm{i}}{ }^{0}$ der entsprechenden freien Tetraeder. Die Bedeutung des Parameters S ist in der Abbildung 5 veranschaulicht. Dort sind beispielhaft für drei verschiedene Tetraeder $\mathrm{i}=1,2$ und 3 die nach Gleichung (30) zu berechnenden Volumina $\mathrm{V}_{\mathrm{i}}$ für verschiedene Werte von $\mathrm{S}$ zu sehen. Für die freien Tetraeder gilt: $\mathrm{V}_{1}{ }^{0}<<\mathrm{V}><\mathrm{V}_{2}{ }^{0}<\mathrm{V}_{3}{ }^{0}$. Die $\mathrm{S}-$ Werte sind den Dreiecken einbeschrieben und durch die verschiedene Graustufen einfach zu unterscheiden:
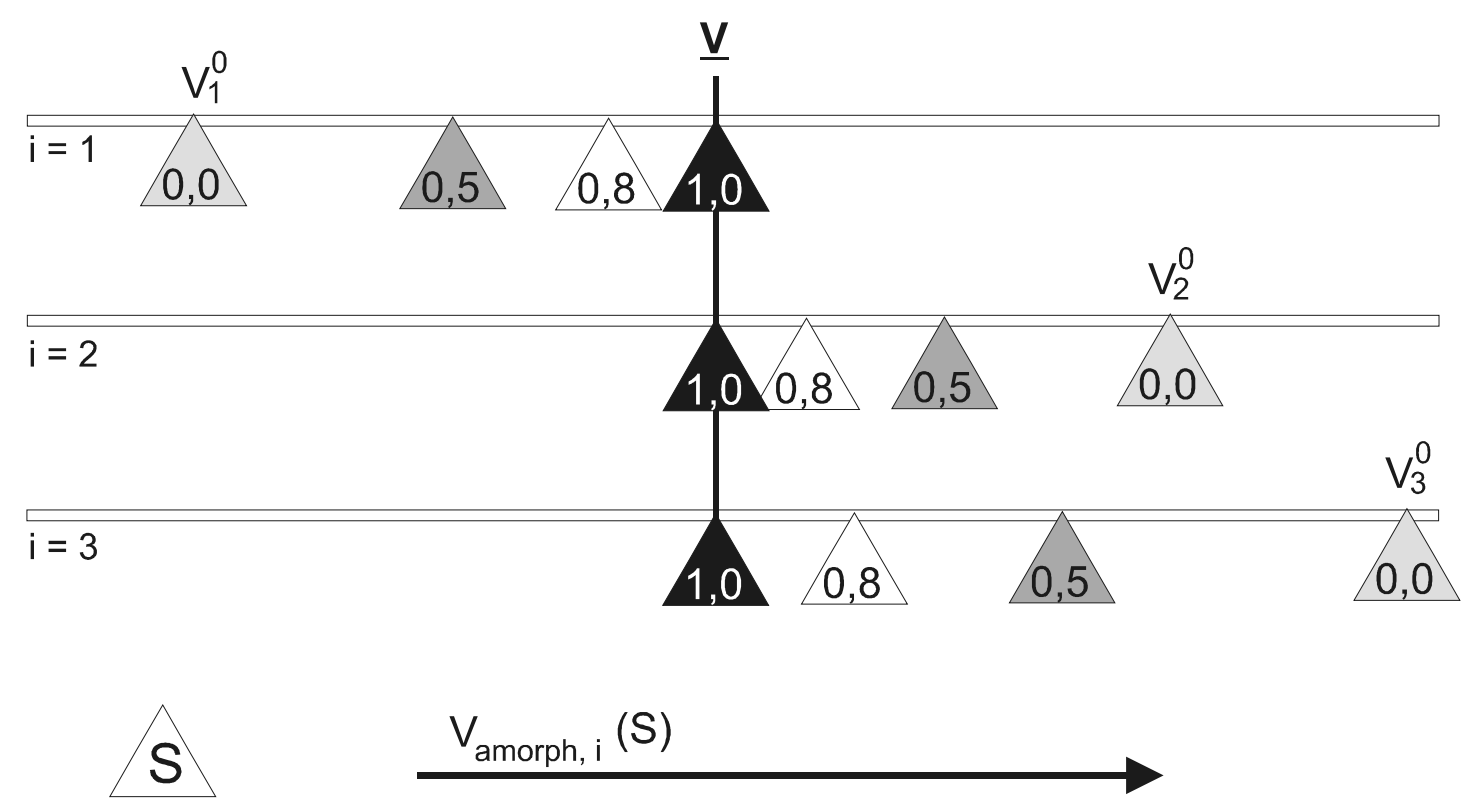

Abbildung 5: Einfluss des Parameters $S$

Ein zunehmender Parameter S bewirkt also eine Verschiebung der Tetraedervolumina auf das mittlere Volumen $<\mathrm{V}>\mathrm{zu}$. Kleine Tetraeder werden gedehnt, während große Tetraeder gestaucht werden. $\mathrm{S}=0,5$ führt beispielsweise dazu, dass die betrachteten Tetraedergrößen der Tetraeder $\mathrm{i}$ genau "in der Mitte" zwischen $\langle\mathrm{V}\rangle$ und $\mathrm{V}^{0}{ }_{\mathrm{i}}$ liegen. Die absoluten Volumenänderungen der einzelnen Tetraeder können dabei vollkommen unterschiedlich sein. Mit diesem Ansatz hat man dann die Möglichkeit gefunden die mittleren Volumina der Tetraedertypen in einer beliebigen Legierung abzuschätzen. 
Um die elastischen Energiebeiträge $\mathrm{zu}$ berechnen, die aus den in Gleichung (30) berechenbaren Tetraederplatzgrößen resultieren, wird im folgenden der Wasserstoff als ideale Kugel mit dem Volumen $V_{K}$ angenommen. Der Tetraederplatz i, auf dem sich der Wasserstoff befindet, wird ebenfalls ideal kugelförmig angenommen und besitzt das Volumen $V_{\text {Loch }}$. Außerdem wird angenommen, dass er sich bezüglich der elastischen Eigenschaften in einer homogenen isotropen Matrix mit dem Schermodul $\eta$ befindet. Die elastische Energie, die benötigt wird, um den Wasserstoff auf den Tetraederplatz i zu setzen, kann somit nach Eshelby [Esh56] mit der Gleichung (31) berechnet werden. Dabei ist zu beachten, dass das Wasserstoffatomvolumen größer als das des zu besetzenden Tetraederplatzes ist.

$$
E_{K, L}=\frac{2}{3} \eta \cdot \frac{\left(V_{K}-V_{\text {Loch }}\right)^{2}}{V_{\text {Loch }}}
$$

Der Wasserstoff findet den Tetraeder $\mathrm{i}$ in der amorphen Legierung nicht in der Größe $\mathrm{V}^{0}{ }_{\mathrm{i}}$ vor, sondern in der aus der Gleichung (30) folgenden Größe $\mathrm{V}_{\mathrm{i}}$. Im Vergleich zum freien Tetraeder tritt also eine zusätzliche konzentrationsabhängige Verzerrungsenergie $\Delta \mathrm{E}_{\mathrm{i}}$ auf.

$$
\Delta E_{i}\left(V_{i}^{0}, V_{i}\right)=\frac{2}{3} \eta\left(\frac{\left(V_{K}-V_{i}^{0}\right)^{2}}{V_{i}^{0}}-\frac{\left(V_{K}-V_{i}\right)^{2}}{V_{i}}\right)
$$

bzw.

$$
E_{i, \text { Lö }}^{0}=E_{i, f r e i}^{0}+\Delta E_{i}\left(V_{i}^{0}, V_{i}\right)
$$

Die Lösungsenergie des Wasserstoffes in einem freien Tetraeder $\mathrm{i}$ beziehungsweise des Tetraeders $\mathrm{i}$ in seiner Referenzlegierung berechnet sich analog zu Gleichung (6) unter der Annahme, dass in den $\mathrm{E}_{\mathrm{j}}^{0}$ die elastischen Energiebeiträge für den freien Tetraeder bereits enthalten sind.

$$
E_{i, f r e i}^{0}=\frac{1}{4} \cdot \sum_{j=1}^{m} n_{i j} E_{j}^{0}
$$


Mit Hilfe dieser Abschätzung können nun die mittleren Energien der Gaußverteilungen, die schließlich die amorphe Struktur der Legierung berücksichtigen, in Gleichung (7) berechnet werden. Zusammen mit den Wahrscheinlichkeiten $\mathrm{W}_{\mathrm{i}}$ aus Gleichung (20) kann die Zustandsdichte $\mathrm{Z}(\mathrm{E})$ für die Wasserstofflösung in einer $\mathrm{m}$-komponentigen Legierung berechnet werden.

\subsubsection{Kurzreichweitige Wechselwirkungen - Blockieren von Tetraederplätzen}

Befindet sich ein Wasserstoffatom auf einem Tetraederplatz so kann er einen benachbarten Tetraederplatz aufgrund elektrostatischer Wechselwirkungen blockieren. Dies ist immer dann der Fall, wenn der Abstand zweier Wasserstoffatome geringer als 0,21nm ausfällt [Swi78]. Jeder Tetraeder besitzt vier Nachbartetraeder. Durch die gemeinsamen Seiten ist die mögliche Zusammensetzung dieser Nachbartetraeder aber eingeschränkt. Befindet sich ein Wasserstoffatom in einem Tetraeder mit der Zusammensetzung $\mathrm{A}_{3} \mathrm{~B}_{1}$, so können nur Tetraeder vom Typ $A_{4}, A_{3} B_{1}$ und $A_{2} B_{2}$ blockiert werden, Tetraederplätze vom Typ $A_{1} B_{3}$ oder $\mathrm{B}_{4}$ existieren jedoch nicht als Nachbartetraeder und können somit auch nicht geblockt werden. Dies resultiert aus der Tatsache, dass sich diese nahen Tetraederplätze, die Seite an Seite liegen zu zweit jeweils drei Atome teilen müssen. Zur Veranschaulichung wurde dieser Sachverhalt in Abbildung 6 noch einmal schematisch für den zwei dimensionalen Fall mit Dreiecken statt Tetraedern dargestellt.

Die Prozedur zur Berechnung von $\mathrm{Z}(\mathrm{E})$ sieht so aus, dass zunächst die energetisch tiefliegenden Tetraeder besetzt und dann deren Nachbarn für weitere Besetzungen nach dem obigen Prinzip ausgeschlossen werden. So blockierte Plätze werden durch eine entsprechende Verringerung von $\mathrm{W}_{\mathrm{i}}$ aus der Zustandsdichte entfernt. Nun werden die nächsten energetisch niedrigsten Plätze der verbleibenden $Z(E)$ aufgefüllt. Mit Hilfe dieses Ansatzes wurden resultierende effektive Zustandsdichten in dieser Arbeit berechnet. Da alle in dieser Arbeit dargestellten berechneten Z(E) eigentlich effektive Zustandsdichten sind, und diesen Effekt des Wasserstoffblocking berücksichtigen, werden diese nach wie vor mit $\mathrm{Z}(\mathrm{E})$ benannt. 


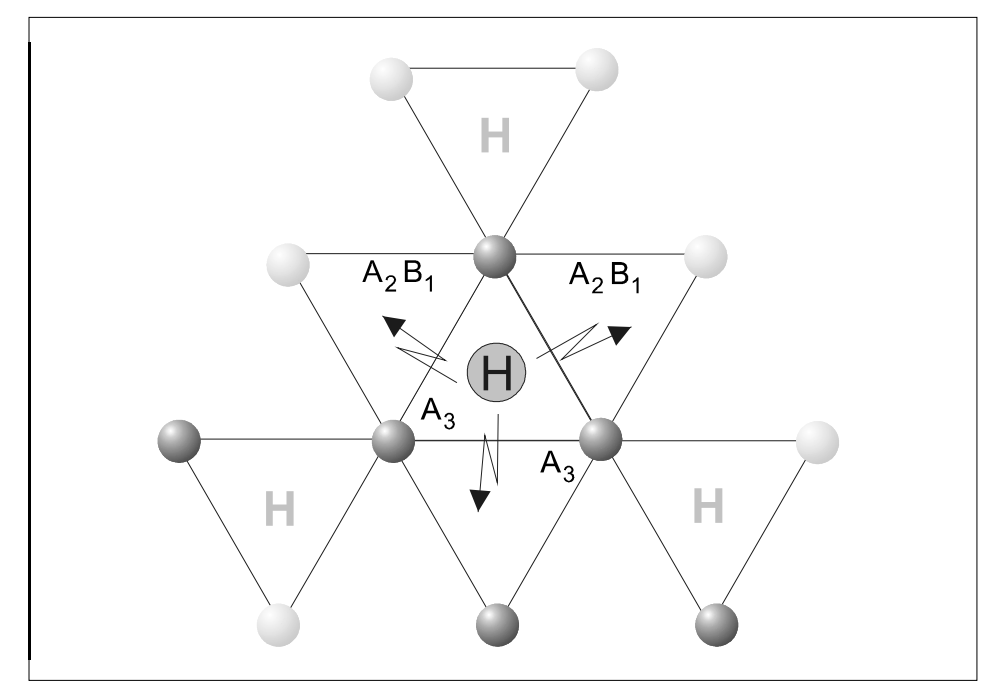

Abbildung 6: Blockieren von Nachbarplätzen zur Veranschaulichung im 2 -dim. Fall : Ein Wasserstoffatom auf einem bestimmten Platz blockiert nur die Plätze, die Seite an Seite mit dem besetzten Platz liegen. Die Zusammensetzung der blockierten Tetraeder ist damit eingeschränkt. Im dargestellten Fall werden keine $A_{1} B_{2}$ und $B_{3}-$ Plätze blockiert.

In der Abbildung 7 ist der Effekt der kurzreichweitigen repulsiven Wasserstoff-WasserstoffWechselwirkung auf $\mathrm{Z}(\mathrm{E})$ in einer $\mathrm{A}_{0,75} \mathrm{~B}_{0,25}$-Legierung dargestellt (die Energien der reinen Elemente sind $\mathrm{E}_{\mathrm{A}}^{0}=-40 \mathrm{~kJ} / \mathrm{mol}$ und $\mathrm{E}_{\mathrm{B}}^{0}=0 \mathrm{~kJ} / \mathrm{mol}$, die Halbwertsbreite aller Verteilungen wurde zu $\sigma=5 \mathrm{~kJ} / \mathrm{mol}$ gewählt). Zuerst werden die tiefliegenden Plätze besetzt. Mit zunehmender Besetzung werden immer mehr Plätze nach der obigen Prozedur blockiert. Die resultierende effektive Zustandsdichte hat daher eine ganz andere Form, als die Zustandsdichte, die man ohne Berücksichtigung dieser Wechselwirkung erhält. Berechnet man die Flächen unter beiden Kurven, so erhalt man 1 für die (normierte) Zustandsdichte ohne Blockieren und 0,45 für die mit Blockieren erhaltene Zustandsdichte. Die früher gemachten Annahmen, dass nur ca. die Hälfte aller möglichen Plätze aufgrund solcher Wasserstoffblockingeffekte besetzt werden [Kir88], ist somit qualitativ richtig. 


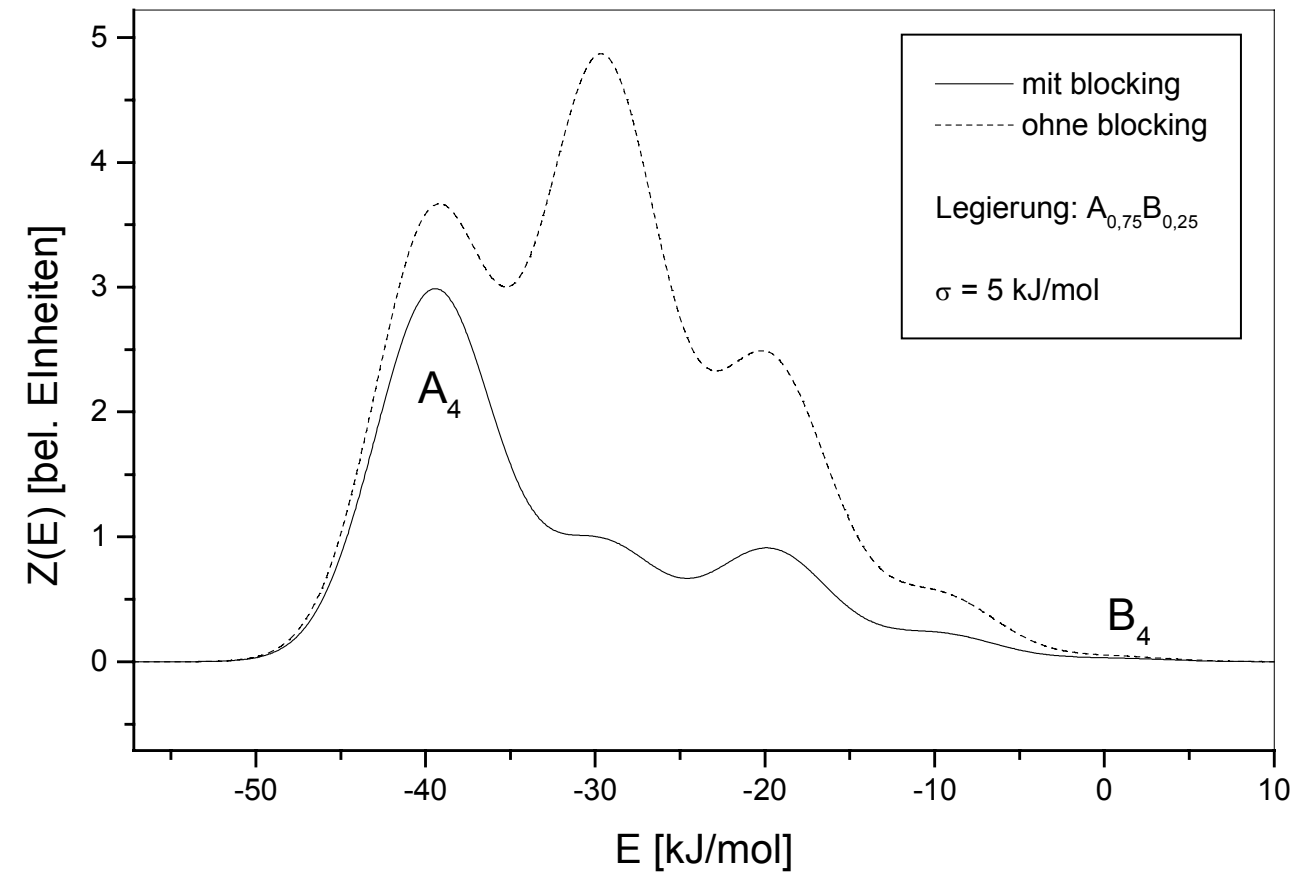

Abbildung 7: Blockieren von Nachbarplätzen am Beispiel einer $A_{0,75} B_{0,25}$-Legierung.Deutlich ist die Veränderung der Zustandsdichte zu erkennen. Nachdem energetisch tiefliegende Plätze besetzt werden, werden immer mehr der verbleibenden Tetraeder blockiert. 


\section{Probenherstellung und experimentelle Methoden}

\subsection{Herstellung und Charakterisierung amorpher Legierungsschichten aus}

$$
\mathrm{Zr}-\mathrm{Ti}-\mathrm{Cu}-\mathrm{Ni}-\mathrm{Be} \text { (Vitreloy) und } \mathrm{Zr}-\mathrm{Al}-\mathrm{Ni}-\mathrm{Cu} \text { (ZANC) }
$$

In dieser Arbeit wurden zwei Proben aus zwei verschiedenen Vitreloy-Legierungen untersucht. Zum einen die sogenannte Vitreloy 1 - Legierung mit der Zusammensetzung $\mathrm{Zr}_{41} \mathrm{Ti}_{14} \mathrm{Cu}_{12.5} \mathrm{Ni}_{10} \mathrm{Be}_{22.5}$ [Pek93]. Zum anderen die sogenannte Vitreloy 4 - Legierung mit der Zusammensetzung $\mathrm{Zr}_{46.75} \mathrm{Ti}_{8.25} \mathrm{Cu}_{7.5} \mathrm{Ni}_{10} \mathrm{Be}_{27.5}$ [Gey96]. Diese Legierungen wurden mittels Sputterdeposition auf einem Si - Substrat (10mm x 10mm) deponiert. Die Schichten waren $150 \mathrm{~nm}$ dünn und wurden zum Schutz vor Oxidation und zur Katalyse des Wasserstoffes [Za195] mit einer ca. 50nm dicken Pd-Schicht, ebenfalls per Sputterdeposition, überzogen. Die Proben wurden freundlicherweise auf Anfrage im HahnMeitner-Institut in Berlin hergestellt. Die amorphe Struktur der so hergestellter Proben wurde mit Röntgenbeugung und Elektronenstrahlmikroskopie bestätigt [Wan00].

Die $\mathrm{Zr}-\mathrm{Al}-\mathrm{Ni}-\mathrm{Cu}$ - Proben wurden im Institut für Materialphysik in Göttingen durch Sputterdeposition gefertigt. Es war das Ziel die amorphe Inuoe-Legierung mit der Zusammensetzung $\mathrm{Zr}_{69.5} \mathrm{Al}_{11} \mathrm{Ni}_{7.5} \mathrm{Cu}_{12}$ mittels Sputterdeposition herzustellen [Zha91]. Dazu wurde ein Sputtertarget verwendet, das aus einer Zr-Platte bestand, auf die Kreissegmente (Fläche $\mathrm{A}_{\mathrm{i}}$ und Öffnungswinkel $\alpha_{\mathrm{i}}$ ) aus den Materialien $\mathrm{Al}, \mathrm{Ni}$ und $\mathrm{Cu}$ mittels eines bonding-Klebers fixiert wurden. Die Zusammensetzung der schließlich deponierten Legierung ergibt sich aus der Abtragsrate $R_{i}$ einzelner Komponenten und deren Flächenanteil $\mathrm{A}_{\mathrm{i}}$ am Target. Für die Konzentration der Komponente X folgt dann:

$$
X_{i}=\frac{R_{i} A_{i}}{\sum_{j} R_{j} A_{j}}=\frac{Y_{i} \alpha_{i}}{\sum_{j} Y_{j} \alpha_{j}}
$$


Dabei gibt der sogenannte Yield $\mathrm{Y}_{\mathrm{i}}$ an, wie viele Atome ein einfallendes Ar-Ion aus dem Target auslöst. Da das Target mit einem Ionenstrahl der Energie $800 \mathrm{eV}$ unter dem Einfallswinkel $35^{\circ}$ abgetragen wurde, wurde der Yield der einzelnen Elemente für diese Parameter mittels einer Monte-Carlo-Simulation (TRIM2000) [Bie80, Zie85, Nas96] bestimmt. Die so ermittelten Kenngrößen sind in Tabelle 3 zusammengefasst.

Tabelle 3: Zur Berechnung des Targetaufbaus notwendigen Daten mit dem Ziel eine $\mathrm{Zr}_{69.5} \mathrm{Al}_{11} \mathrm{Ni}_{7.5} \mathrm{Cu}_{12}$-Legierung herzustellen.

\begin{tabular}{|c|c|c|c|c|}
\hline & $\mathbf{Z r}$ & Al & $\mathbf{N i}$ & $\mathbf{C u}$ \\
\hline Sputterrate $\mathrm{Y}_{\mathrm{i}}$ [Atom/Ar-Ion] & 1,40 & 3,83 & 3,26 & 4,10 \\
\hline relative Sputterrate $Y_{i \text {, rel }}$ & 0,111 & 0,304 & 0,259 & 0,326 \\
\hline gewünschte Konzentration $\mathrm{c}_{\mathrm{i}}[\mathrm{at} \%]$ & 69,5 & 11,0 & 7,5 & 12,0 \\
\hline Winkelverhältnis $\mathrm{I}_{\alpha \mathrm{i}}$ & 0,860 & 0,050 & 0,040 & 0,051 \\
\hline Winkel $\left[{ }^{\circ}\right] \alpha_{i}$ & 309,5 & 17,9 & 14,3 & 18,3 \\
\hline
\end{tabular}

Mit der so berechneten Winkel-bzw. Flächenverteilung des Targets wurden dünne Schichten der Legierung auf Si-Substraten hergestellt. Die Schichtdicken der in dieser Arbeit verwendeten Proben lagen zwischen $50 \mathrm{~nm}$ und $200 \mathrm{~nm}$. Zum Schutz vor Oxidation und zur Katalyse des Wasserstoffes [Za195] wurden auch diese Proben mit einer ca. $50 \mathrm{~nm}$ dicken Pd-Schicht überzogen. Die Fläche der Proben zur Isothermenmessung lag bei ca. $100 \mathrm{~mm}^{2}$ bis $200 \mathrm{~mm}^{2}$.

Die Abbildung 8-a zeigt die Konzentrationen an sechs verschiedenen Probenstellen auf einer so hergestellten Probe, wie sie mittels EDX (Energy Dispersive $\underline{X}$-ray Microanalysis) gemessen wurden. 

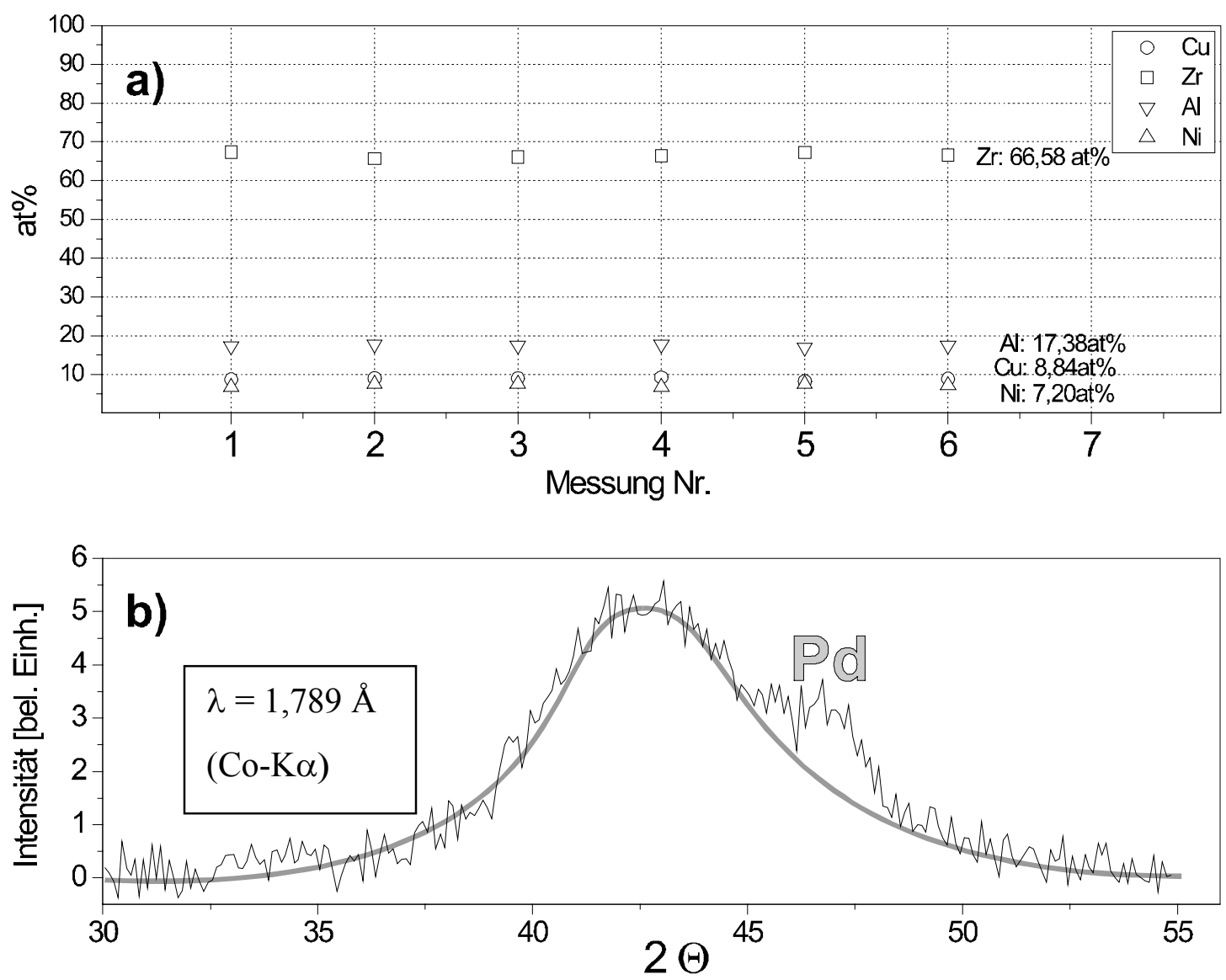

Abbildung 8: EDX und Röntgenuntersuchungen zur Bestimmung der Zusammensetzung und Struktur der amorphen $\mathrm{Zr}$-Ni-Al-Cu-Proben.

Die Zr-Ni-Al-Cu-Legierung war offensichtlich über die Probenfläche homogen und entsprach der Zusammensetzung: $\mathrm{Zr}_{66,8} \mathrm{Al}_{17,4} \mathrm{Ni}_{7,2} \mathrm{Cu}_{8,8}$. Diese Zusammensetzung entspricht also näherungsweise der der gewünschten Legierung. Kleinere Abweichungen waren bei dem hier verwendeten Verfahren jedoch auch aus den folgenden Gründen zu erwarten: a) Das Yield wird mittels TRIM nur für amorphe Targets berechnet. Polykristalline Targets zeigen eine im Vergleich dazu kleinere Abtragsrate. b) Das abgetragene Material weist eine von der Atommasse abhängige Vorzugsflugrichtung vom Target weg auf [Ben87]. c) Die Ionendichte des abtragenden Ar-Strahls ist über dem Target nicht konstant [Ban98]. 
Mit Hilfe des Röntgendiffraktogramms in Abbildung 8-b konnte die amorphe Struktur der Proben durch den breiten Peak zwischen $2 \Theta=35^{\circ}$ und $2 \Theta=50^{\circ}$ identifiziert werden. Zusätzlich zeigt Abbildung 8-b bei $2 \Theta \approx 46^{\circ}$ den $(111)$ - peak der kristallinen Pd Deckschicht. 


\subsection{Experimentelle Methoden}

Dieses Kapitel stellt die experimentellen Methoden vor, mit denen das chemische Potential einer Probe in Abhängigkeit von der Konzentration des gelösten Wasserstoffes gemessen wird. Für das chemische Potential einer Probe gilt:

$$
\mu_{H}=\mu_{0, H}+\frac{R T}{2} \ln \frac{p_{H_{2}}}{p_{H_{2}, 0}}
$$

Das chemische Potential des Wasserstoffes bei dem frei wählbarem Referenzzustand $\mathrm{T}=25^{\circ} \mathrm{C}$ und $\mathrm{p}_{\mathrm{H} 2,0}=1$ bar beträgt dabei $\mu_{0, \mathrm{H}}=0 \mathrm{~kJ} / \mathrm{mol}$. Die Bestimmung des chemischen Potentials kann neben einer Messung des Partialdruckes $\mathrm{p}_{\mathrm{H} 2}$ und Gleichung (36) auch elektrochemisch geschehen. Dabei wird die elektromotorische Kraft (EMK) als Potentialdifferenz U der zu untersuchenden Probe und einer Referenzelektrode gemessen. Den Zusammenhang zwischen der EMK und dem Wasserstoffdruck liefert die Nernstsche Beziehung:

$$
U=U_{0}-\frac{R T}{2 F} \ln \frac{p_{H_{2}}}{p_{H 2,0}}
$$

mit

$$
\begin{aligned}
& F=96.485 \mathrm{C} / \mathrm{mol} \quad(\text { Faraday-Konstante) } \\
& \text { und } \\
& R=8,314 \mathrm{~J} / \mathrm{K} \cdot \mathrm{mol} \text { (Gaskonstante) }
\end{aligned}
$$

Mit Gleichung (36) folgt die Beziehung zwischen EMK und dem chemischen Potential:

$$
\mu_{H}(U)=-\left(U-U_{0}\right) F
$$

Die Referenz - EMK $\mathrm{U}_{0}$ ist wieder auf den Referenzzustand $\mathrm{T}=25^{\circ} \mathrm{C}$ und $\mathrm{p}_{\mathrm{H} 2,0}=1 \mathrm{bar}$ bezogen, hängt aber auch von den Eigenschaften der zweiten Elektrode ab. Zu Beginn von EMK - Messungen wird $U_{0}$ durch Referenzmessungen experimentell bestimmt. 
Um die experimentell bestimmten Isothermen $\mu\left(\mathrm{c}_{\mathrm{H}}\right)$ mit den Ergebnissen der theoretischen Überlegungen aus Kapitel 2.3 vergleichen zu können, wurden die $\mu\left(c_{H}\right)-$ Verläufe in Zustandsdichten umgerechnet. Dabei ergibt sich die Zustandsdichte entsprechend Gleichung (4) direkt aus der Differentiation der Messdaten $U\left(\mathrm{c}_{\mathrm{H}}\right)$ in der achsenvertauschten Darstellung $\mathrm{c}_{\mathrm{H}}(\mathrm{U})$. Die so erhaltene Zustandsdichte $\mathrm{Z}(\mathrm{U})$ wird mit Hilfe von Gleichung (38) in die Form $Z(\mu)$ bzw. $Z(E)$ umgerechnet.

\subsubsection{Isotherme elektrolytische Wasserstoffbeladung dünner Schichten}

Die amorphen Proben wurden elektrochemisch mit Wasserstoff beladen. Die Schichten wurden mit Drähten kontaktiert, indem diese mit Leitsilber auf die Probenoberfläche geklebt wurden. Die Kontaktstelle wurde mit einem handelsüblichen 2-Komponenten Kleber isoliert. Sowohl die zu beladene Probe, eine Arbeitselektrode ( $\mathrm{Pt}-\mathrm{Draht}$ ) und eine $\mathrm{Ag} / \mathrm{AgCl}-$ Referenzelektrode befanden sich in einem Elektrolyten. Der verwendete Elektrolyt bestand zu einem Drittel aus 85\%iger Phosphorsäure und zu zwei Dritteln aus reinem Glycerin [Kir80]. Er wurde durch Argongas von gelöstem Sauerstoff gereinigt. Konstante Temperaturen wurden durch ein Ölbad, in dem sich das Gefäß befand, realisiert.

Zur Beladung der Probe floss für eine bestimmte Zeit t ein Strom der Stärke I zwischen der Probe und der auf positiveren Potential liegenden Arbeitselektrode. Dabei werden auf der leitenden Probenoberfläche $\mathrm{H}^{+}$- Ionen des Elektrolyten durch Aufnahme von Elektronen aus der Probe neutralisiert, lösen sich in der Probe und diffundieren, auch nach Abschalten des Stromes, in die Probe. Die Änderung der Gesamtwasserstoffkonzentration $\Delta c_{H}$ in der Probe durch jeden Stromstoß kann mit Hilfe des Faraday-Gesetzes berechnet werden:

$$
\Delta c_{H}=\frac{\Delta n_{H}}{n_{M e}}=\frac{Q}{F} \cdot \frac{V_{\text {mol }}}{V_{\text {probe }}}=\frac{I \cdot t}{F} \cdot \frac{V_{\text {mol }}}{V_{\text {probe }}}
$$

Dabei ist $\Delta \mathrm{n}_{\mathrm{H}}$ die Molzahl des aufgenommenen Wasserstoffes und $\mathrm{n}_{\mathrm{Me}}$ die Molzahl der Metallatome in der Probe mit dem Volumen $\mathrm{V}_{\text {probe }}, \mathrm{V}_{\text {mol }}$ das Molvolumen der Legierung und F die Faraday-Konstante. In allen Messungen in dieser Arbeit wurde darauf geachtet, dass 
die Stromdichte beim Beladen einer Probe den Wert von $\mathrm{j}=0,5 \mathrm{~mA} / \mathrm{cm}^{2}$ nicht überschreitet. Erfahrungen haben gezeigt, dass höhere Stromdichten zu einer Wasserstoffbläschenbildung an der Probenoberfläche führen können. Die berechneten Konzentrationen wären dann geringer. Die verwendeten Stromstärken lagen bei ca. 0,07 mA und die Zeit, für die sie flossen, lag zwischen einer Sekunde und mehreren Minuten.

Bis zum Erreichen des thermodynamischen Gleichgewichtes verringert sich mit der HKonzentration an der Oberfläche auch das chemische Potential und damit nach Gleichung (38) auch die Elektromotorische Kraft (EMK). Die EMK wurde als elektrische Spannung zwischen der Probe und einer Ag/AgCl - Elektrode gemessen. Die Referenz - EMK wurde bei den in dieser Arbeit verwendeten Elektroden $\mathrm{zu}_{0}=-182 \mathrm{mV}$ bestimmt. Diesem Wert ist das chemische Potential $\mu_{0, \mathrm{H}}\left(1 \mathrm{bar}, 25^{\circ} \mathrm{C}\right)=0 \mathrm{~kJ} / \mathrm{mol}$ zugeordnet.

Die Spannungsmessung erfolgte mittels eines Impedanzwandlers stromlos, um durch die Spannungsmessung bedingte Wasserstoffbe- bzw. entladungen zu verhindern. Die Spannung wurde mit einem Messgerät der Fa. Keythley aufgenommen und über eine IEEE - Verbindung von einem PC aufgezeichnet. Der gesamte Versuchsaufbau ist in der Abbildung 9 dargestellt.

Die Beladung der Vitreloy - Proben und einer ZANC - Probe wurde mit einer Zeitschaltuhr durchgeführt, die nach manueller Auslösung ein Relais für eine vorgegebene Zeit schloss und den gewünschten Strom zur Wasserstoffbeladung durch Probe und Gegenelektrode fließen ließ. Zwei Beladungen an ZANC-Proben wurden mit einer automatischen Zeitschaltuhr durchgeführt. Diese erlaubte es, exakte Zeitintervalle zwischen den einzelnen Beladungen zu realisieren. Der Grund hierfür war die Idee, etwaige systematische Fehler, die bei der Isothermenbestimmung eine Rolle spielen können, zu reduzieren. Mit einer Zeitschaltuhr, die konstante Pausen zwischen zwei Beladungen läßt, besteht die Möglichkeit, dass z.B. bei einer konstant angenommenen Drift in der EMK (durch Wasserstoffverluste) für alle Messpunkte der gleiche Fehler entsteht.

Die automatische Zeitschaltuhr kam bei zwei ZANC-Probe mit einer Oberfläche von ca. $200 \mathrm{~mm}^{2}$ zum Einsatz. Für jeden Beladungsschritt floss für die Zeit von $\mathrm{t}=30 \mathrm{sec}$ ein Strom von $\mathrm{I}=0,07 \mathrm{~mA}$. Die Zeit zwischen den Beladungsschritten betrug 60 Minuten. 


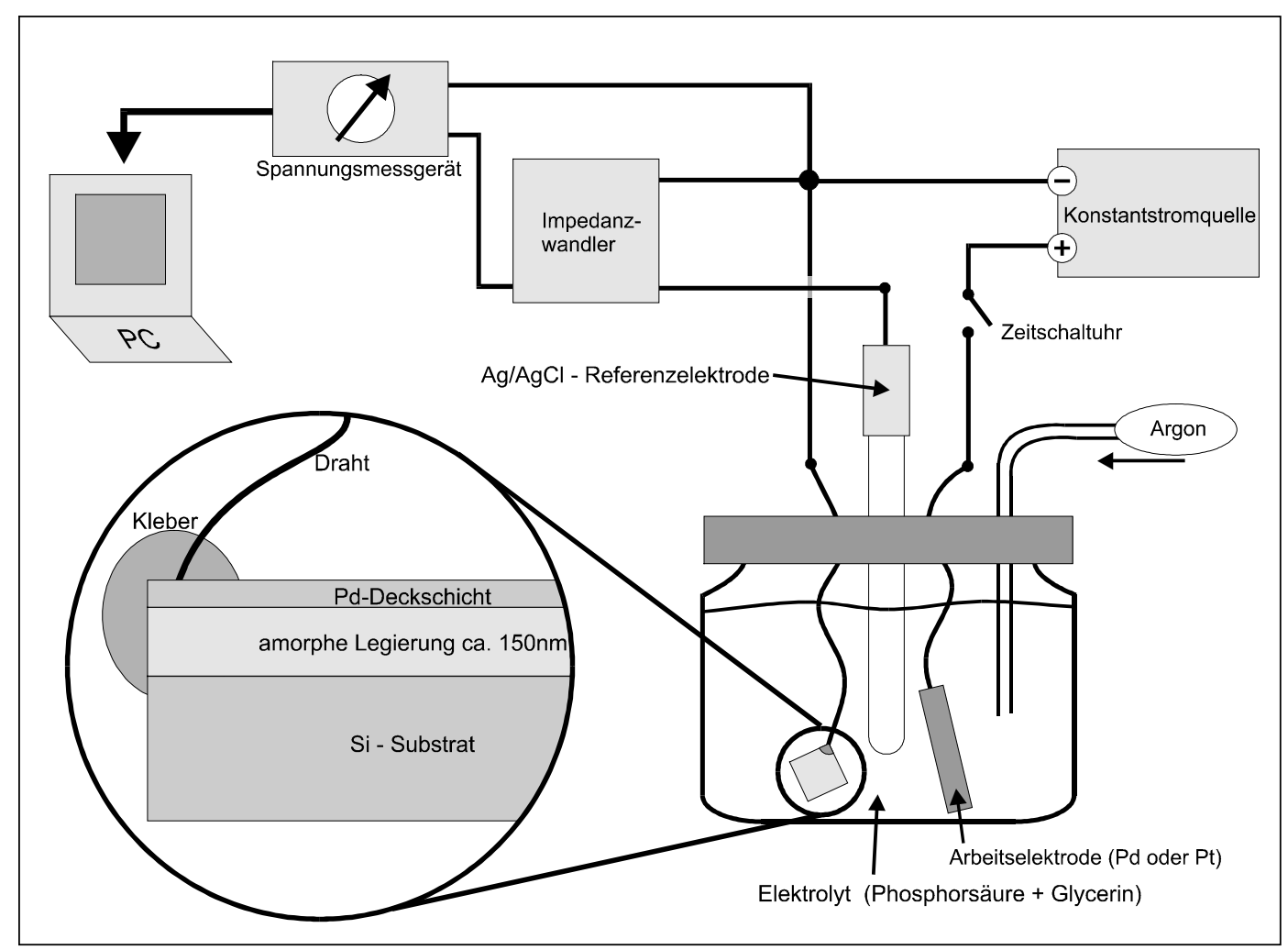

Abbildung 9: Experimenteller Aufbau zur elektrolytischen Wasserstoffbeladung und Probenquerschnitt.

Die Auftragung der im thermodynamischen Gleichgewicht eingestellten EMK gegen die Gesamtkonzentration stellen die Isothermen $U\left(c_{H}\right)$ dar.

\subsubsection{Messung der Wasserstoffdiffusion mit Hilfe der Doppelzelle}

Eine Methode zur quantitativen Bestimmung von Wasserstoffdiffusionskoeffizienten ist die Messung von Durchbruchszeiten in Permeationsexperimenten [Sto84a]. Die zu untersuchende Probe trennt als Membran zwei sich in einem Elektrolyten befindliche Elektroden. Dann wird ein zeitlich konstanter Strom auf der Beladungsseite zwischen Arbeitselektrode und Probe eingeschaltet. Dabei belädt sich die Probenoberfläche und der Wasserstoff diffundiert durch die Probe und erreicht nach einiger Zeit die andere Probenseite, wo er jetzt das chemische Potential verändert. Die zwischen der Probe und 
einer auf der Messseite platzierten Referenzelektrode gemessene EMK verändert sich somit. Der zu erwartende EMK-Verlauf ist in Abbildung 10 schematisch dargestellt. Es ist ein flacher Anstieg zu sehen, der im Laufe der Zeit immer steiler wird und schließlich für große Zeiten, wenn sich die Konzentration in der Probe nicht weiter ändert, einen Sättigungswert annimmt.

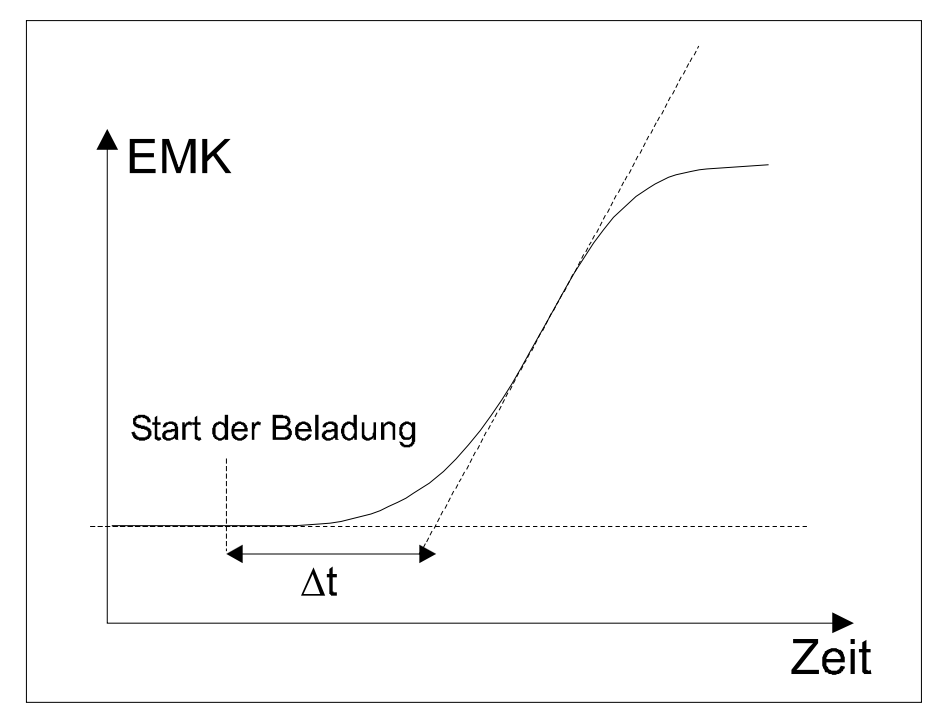

Abbildung 10: Bestimmung der Durchbruchszeit bei Permeationsmessungen.

Die charakteristische Durchbruchszeit $\Delta t$ wird als Differenz zwischen dem Beginn der Beladung und dem Schnittpunkt der Tangente an den Wendepunkt der EMK-Kurve bestimmt. Aus dieser Durchbruchszeit kann der intrinsische Diffusionskoeffizient in einer Schicht der Dicke s berechnet werden [Sto84b, Kir80, Cra67]:

$$
D_{i n}=\frac{s^{2}}{6 \cdot \Delta t}
$$

Bei der Untersuchung der Diffusion durch dünne Schichten können diese auf eine Pd - Trägerfolie, die einige $\mu \mathrm{m}$ dick sein kann, deponiert werden [Kes00]. Dies erhöht die Stabilität und Handhabbarkeit der nur einige $10 \mathrm{~nm}$ dünnen Schichten. Bei den Bestimmungen der Durchbruchszeiten wird die Diffusion durch diese Pd-Trägerfolie entsprechend berücksichtigt. Die in dieser Arbeit untersuchten Materialien sind amorphe 
metallische Gläser. Im Gegensatz $\mathrm{zu}$ den in [Kes00] untersuchten $\mathrm{Pd}-\mathrm{Nb}-$ Schichtsystemen ist es bei den Gläsern aufgrund ihrer Sprödigkeit nicht möglich, Experimente mit Schichten auf einer dünnen und flexiblen Pd-Folie durchzuführen, da diese schon bei einer leichten Verbiegung der Trägerfolie zu Rissbildung neigen. Bei einer anschließenden Messung der Durchbruchszeiten würde lediglich eine Kurzschlussdiffusion durch das Pd gemessen werden. Stabile dicke Pd-Bleche sind ebenfalls als Substrate für Permeationsmessungen an dünnen Schichten ungeeignet, da die Diffusionszeiten durch das Pd drastisch in die Höhe gehen ( $t$ proportional $s^{2}$ !) und der Effekt durch die zusätzliche amorphe Schicht zu klein wird.

Auch die Verwendung von stabileren Proben (einige $100 \mu \mathrm{m}$ ) aus Vitreloy 4 ohne Pd-Trägerfolie als Membran in einer elektrochemischen Doppelzelle lieferte unbefriedigende Ergebnisse [Pun01]. Entweder waren die Durchbruchszeiten zu groß und aufgrund der Drift der Zellspannung nicht erkennbar, oder die Gradienten der Wasserstoffkonzentration führten $\mathrm{zu}$ so großen Gradienten in den mechanischen Spannungen in der Probe, dass diese an der Dichtungsstelle durchriss.

Da also die Doppelzellenmessung mit einer amorphen Probe offensichtlich nicht durchführbar ist, wurde in dieser Arbeit ein neues Verfahren entwickelt, das trotz der Sprödigkeit der zu untersuchenden Schicht Permeationsexperimente aus der Gasphase mittels Widerstandsmessungen ermöglicht. Dies ist im folgenden beschrieben.

\subsubsection{Ein neues Verfahren zu Bestimmung von Durchbruchszeiten für Diffusionsuntersuchungen}

Das in diesem Abschnitt vorgestellte und im Rahmen dieser Arbeit entwickelte Verfahren erlaubt es quantitative Untersuchungen der Wasserstoffdiffusion durch dünne amorphe, auf Si deponierte, Schichten durchzuführen. Dabei wird die Veränderung des elektrischen Widerstandes einer $\mathrm{Pd}-$ Schicht bei Wasserstoffbeladung ausgenutzt. Eine bestimmte Geometrie der Probe ist erforderlich, um im Sinne von Permeationsexperimenten Durchbruchszeiten zu bestimmen. 
In der Abbildung 11 ist zunächst dargestellt, wie sich der Widerstand von $\mathrm{Pd}$ in Abhängigkeit mit dem Wasserstoffgehalt verhält [Fro76] verändert.

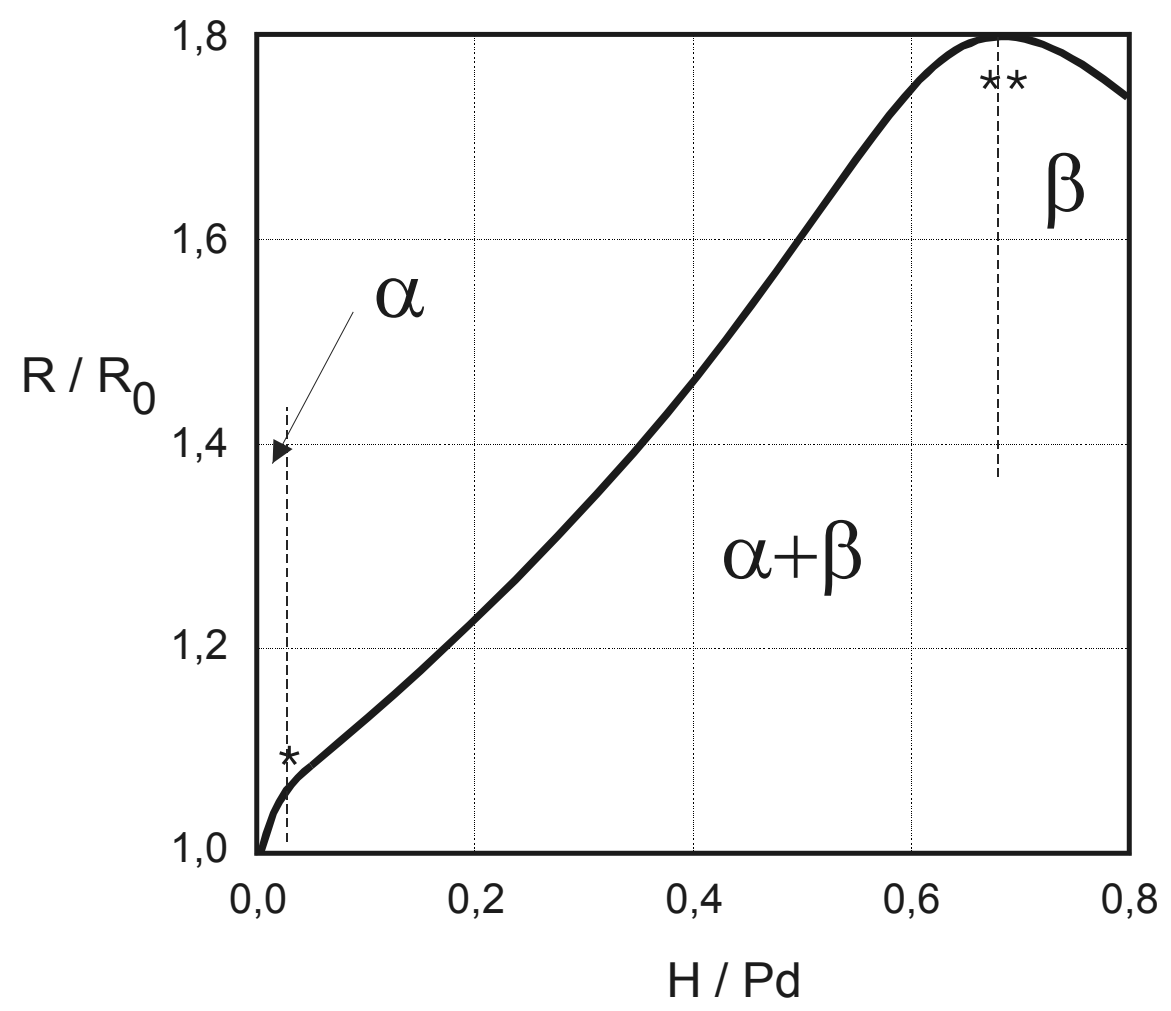

Abbildung 11: Relative Widerstandsänderung von reinem Palladium als Funktion des aufgenommenen Wasserstoffes [Fro76].

Die mit einem bzw. zwei Sternen markierten Konzentrationen begrenzen das Zweiphasengebiet zwischen der $\alpha$ - und der $\beta$ - Phase. Die größte Widerstandsänderung pro Wasserstoffkonzentration wird also in der $\alpha$ - Phase beobachtet.

Um die Wasserstoffdiffusion durch eine dünne amorphe Schicht zu bestimmen, wurde die in Abbildung 12 gezeigte Probengeometrie verwendet: auf einer Pd-Leitschicht befindet sich die $\mathrm{zu}$ untersuchende amorphe metallische Schicht. Diese ist mit einer dünnen PdDeckschicht zur Beschleunigung der Wasserstoffaufnahme und zum Schutz vor Oxidation bedeckt [Za195]. Das Schichtpaket befindet sich auf einem ca. 1mm dicken und stabilen SiSubstrat. 
Pd I (Deckschicht) ca. $<10 \mathrm{~nm}$

\begin{tabular}{|l|}
\hline amorphe Legierung $100 \mathrm{~nm}$ \\
\hline Pd II (Leitschicht) $60 \mathrm{~nm}$ \\
\hline Si-Substrat
\end{tabular}

Abbildung 12: Probengeometrie für Proben zur Durchbruchszeitenbestimmung mittels Widerstandsmessungen mit typischen Schichtdicken.

Abbildung 13-a zeigt das Messprinzip an diesen Schichtsystemen. Die mittels Leitsilber an zwei Stellen kontaktierten Proben werden in einen Rezipienten eingebaut und evakuiert. Gemessen wird der Widerstand der Pd - Leitschicht. Dies erfolgt über die sehr empfindliche Messung mit einer Wheatstoneschen Brückenschaltung. Die gewonnenen Messdaten werden mit einem PC aufgenommen und gespeichert. 
a)

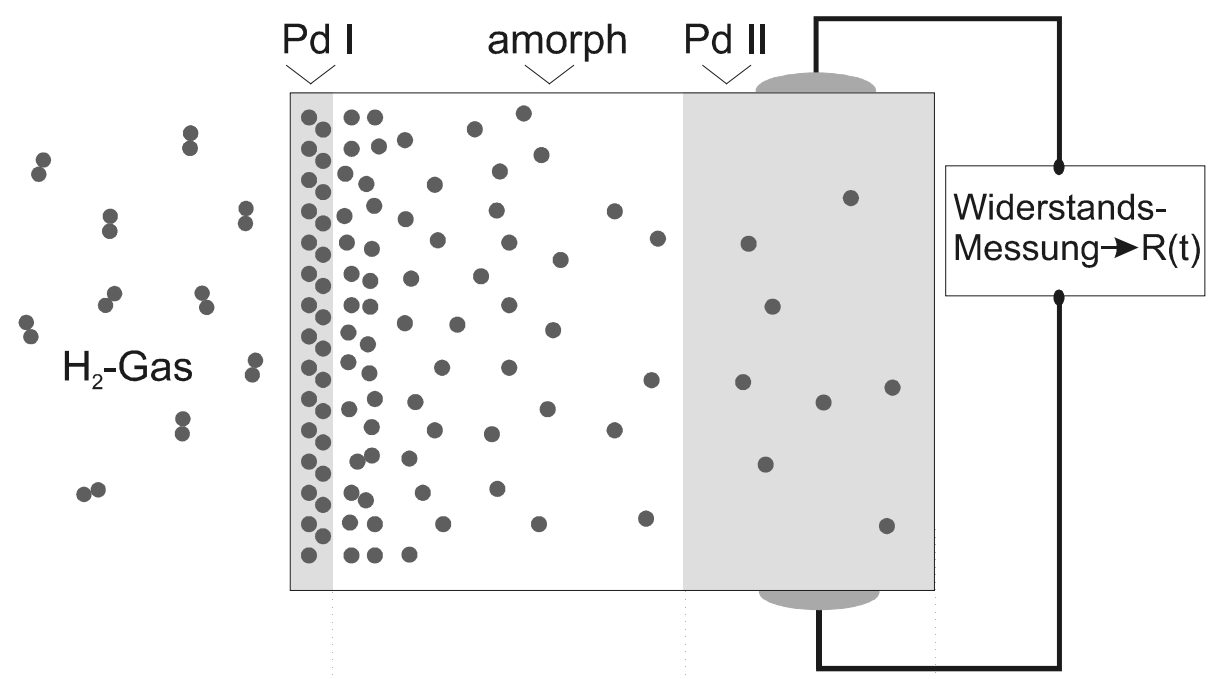

b)

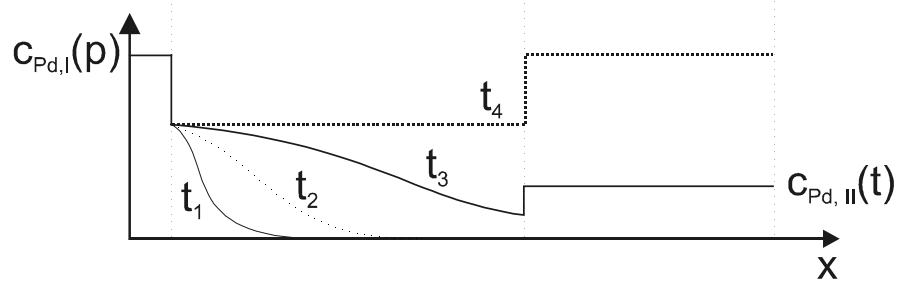

c)

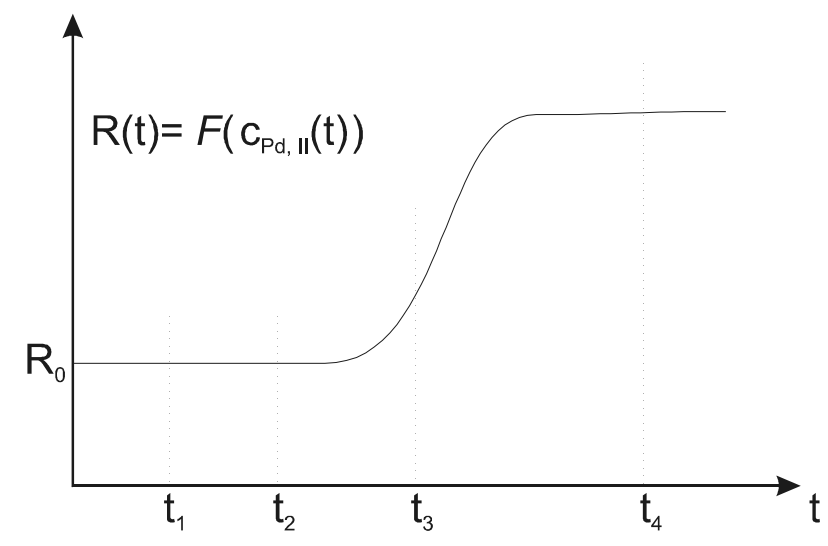

Abbildung 13: a) Prinzip der Wasserstoffaufnahme und-diffusion durch ein Pd/Glas/Pd-Schichtpaket; b) zu erwartende Konzentrationsverläufe in der Probe zu verschiedenen Zeiten; c) der Widerstand dieser Pd-Leitschicht als Funktion der Zeit.

Stellt man in dem Rezipienten einen zeitlich konstanten Wasserstoffdruck ein, belädt sich die Probe aus der Gasphase. Die in dieser Arbeit zur Beladung eingestellten Wasserstoffdrücke waren immer kleiner als 10 mbar. Nach dem Pd-Phasendiagramm 
(s. Anhang 7.1) befindet sich die Probe bei diesen Drücken noch in der $\alpha$ - Phase, womit die nach Abbildung $11 \mathrm{zu}$ erwartenden Widerstandsänderungen kleiner als 5\% sind, aber am empfindlichsten auf eine Wasserstoffkonzentrationsänderung reagieren. Der Wasserstoff diffundiert durch die amorphe Schicht und erreicht schließlich die Pd - Leitschicht wodurch sich deren Widerstand entsprechend Abbildung 11 ändert. In Abbildung 13-b) und c) sind die $\mathrm{zu}$ vier verschiedenen Zeiten zu erwartenden Konzentrationsverläufe in der Probe bzw. der Widerstand der Pd-Leitschicht aufgetragen. Der Vergleich des Wasserstoffdiffusionskoeffizienten in $\mathrm{Pd}\left(\mathrm{ca} . \mathrm{D}_{\mathrm{H}}, \mathrm{Pd}=4 \times 10^{-7} \mathrm{~cm}^{2} / \mathrm{s}\right.$ ) mit Diffusionskoeffizienten an amorphen Materialien bei Raumtemperatur lässt eine um einige Größenordnungen langsamere Diffusion im amorphen Material erwarten [Kie89]. Die in Abbildung 13-c) gezeigten Konzentrationsverläufe, die von einer homogenen Verteilung im Pd ausgehen, sind somit gerechtfertigt. Bestimmt man in Abbildung 13-c) die Durchbruchszeit $\Delta \mathrm{t}$ entsprechend Abbildung 10 so kann mit dieser Methode der Wasserstoffdiffusionskoeffizient mit Hilfe der Gleichung (40) berechnet werden.

Es hat sich gezeigt, dass bei verschiedenen Schichtdicken der Pd-Leitschicht das Verhältnis der Sättigungswiderstände im wesentlichen dem inversen Verhältnis der Pd-Schichtdicken entspricht. Der Einfluss der amorphen Schicht auf den Gesamtwiderstand der Probe ist dabei vernachlässigbar klein, da der spezifische Widerstand eines amorphen Materials wesentlich größer als der des reinen Pd ist und die Widerstände hier als Parallelschaltung miteinander verknüpft sind. Das Verhältnis der spezifischen Widerstände an den hier vorgestellten Proben wurde in Vorversuchen zu $\rho_{\text {amorph }} / \rho_{P d}=40$ bis 50 bestimmt. 


\section{Ergebnisse}

\subsection{Experimentelle Bestimmung von Isothermen und Zustandsdichten Z(E),}

In diesem Abschnitt werden die experimentell bestimmten Isothermen von zwei VitreloyLegierungen und einer ZANC-Legierung vorgestellt. Die Probengeometrien und die Zusammensetzungen entsprachen den in Kapitel 3.1 gemachten Angaben. Um die Zustandsdichte $\mathrm{Z}(\mathrm{U}) \mathrm{zu}$ erhalten, wurden die Verläufe der Isothermen nach achsenvertauschter Auftragung differenziert (vgl. Kapitel 2.1.1).

\subsubsection{Vitreloy-Legierungen}

Abbildung 14 zeigt drei Isothermen, die an einer Vitreloy 4 (Vit 4)-Probe bei Raumtemperatur gemessen wurden. Die angegebenen EMK - Werte beziehen sich noch auf die verwendeten $\mathrm{Ag} / \mathrm{AgCl}$ - Elektroden. Nachdem die erste Wasserstoffbeladung bis zu einer EMK von ca. $-150 \mathrm{mV}$ durchgeführt wurde, wurde die Probe wieder mit einer Entladespannung von $500 \mathrm{mV}$ entladen. Anschließend fanden zwei weitere Beladungen statt. Da die Startkonzentrationen des Wasserstoffes in der Probe unbekannt war, wurden die Kurven so verschoben, dass sie deckungsgleich sind. Die X-Achse gibt also die Zunahme der Wasserstoffkonzentration in Folge der Beladung an. Die Startkonzentration war bei der 2. bzw. 3. Beladung um 0,15 bis 0,20 H/Me größer als bei der ersten Beladung. Bis auf die Verschiebung infolge der Startkonzentration sind die Kurven deckungsgleich, die Be- und Entladung erfolgte reversibel, es traten also keine die Probe verändernden Prozesse, wie Oxidation oder Schichtablösung, auf. Bei Entladespannungen größer als $500 \mathrm{mV}$ wird allerdings eine Oxidation der Probe beobachtet. 
a)

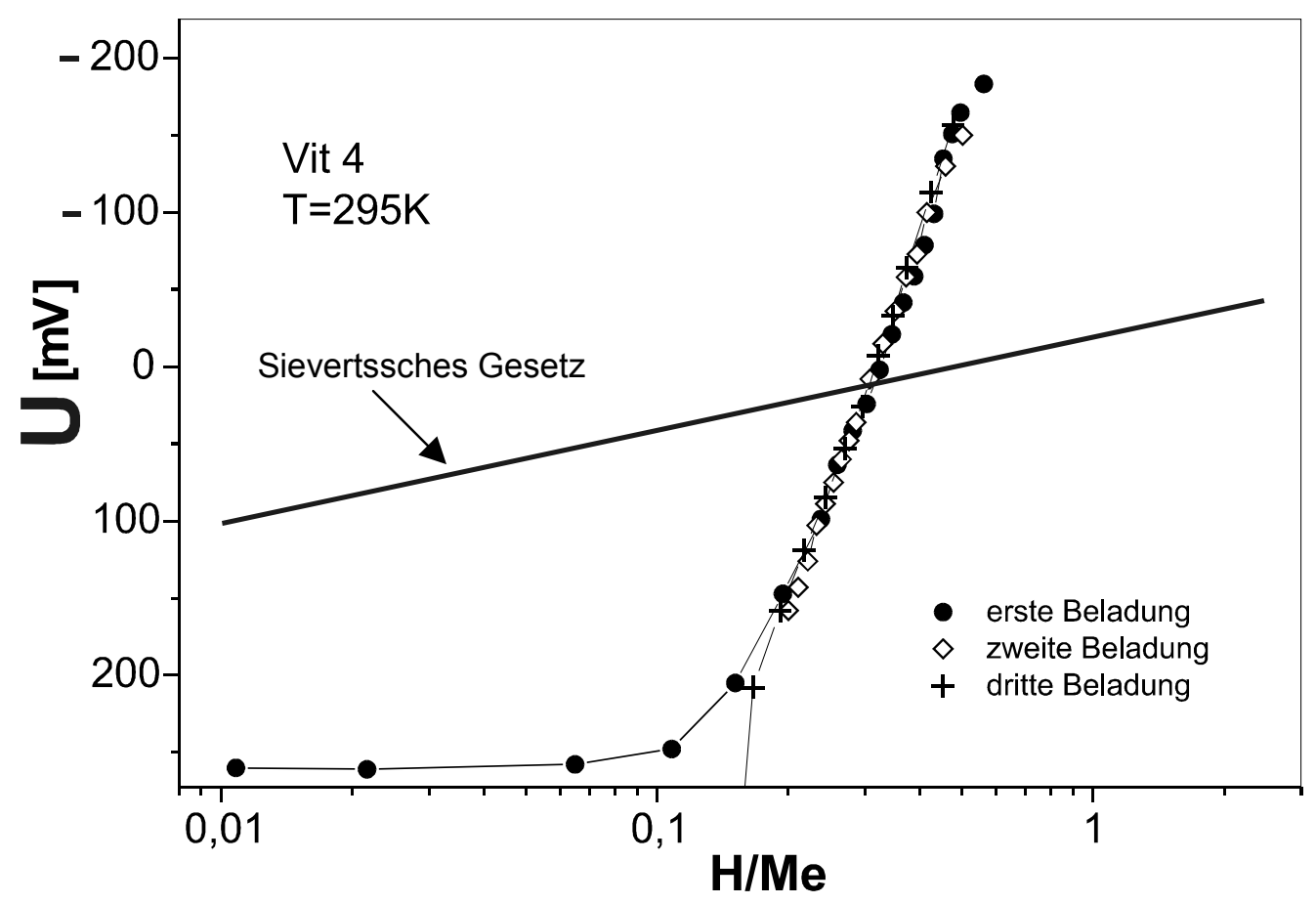

b)

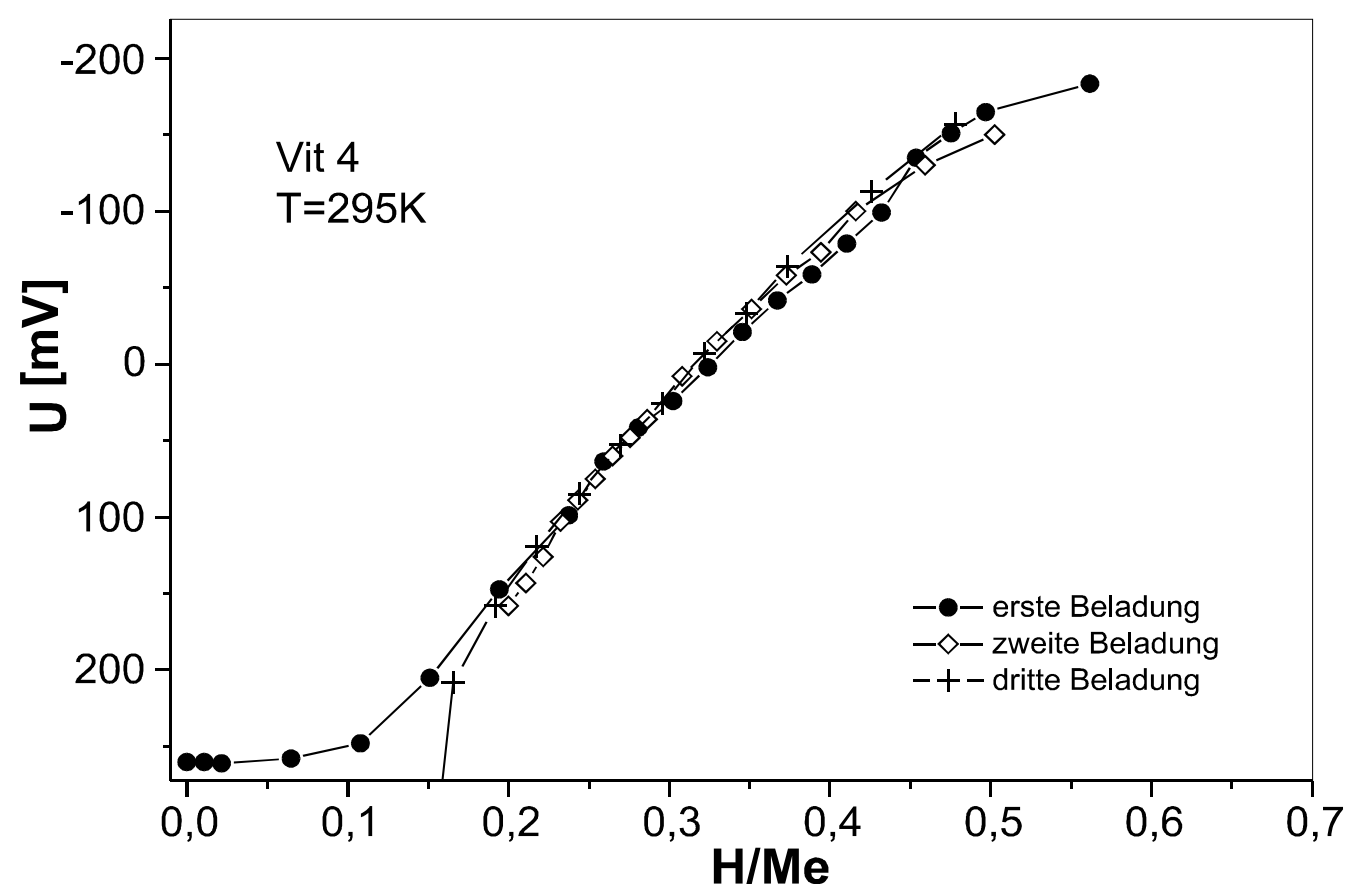

Abbildung 14: Isotherme an der amorphen $\mathrm{Zr}_{46.75} \mathrm{Ti}_{8.25} \mathrm{Cu}_{7.5} \mathrm{Ni}_{10} \mathrm{Be}_{27.5}$. Die EMK wurde in a) gegen den Logarithmus der Konzentration aufgetragen. (Sieverts-Darstellung). Die in a) eingezeichnete Gerade hat die für eine ideal verdünnte Lösung erwartete Steigung. In Darstellung b) wurde die EMK linear gegen die Konzentration aufgetragen. 
Bei Konzentrationen unter $\mathrm{c}_{\mathrm{H}}=0,10 \mathrm{H} / \mathrm{Me}$ ist ein Plateau $\mathrm{zu}$ erkennen. Dieses deutet nach Gleichung (4) an, dass bei einer EMK von ca. $260 \mathrm{mV}$ eine hohe Zustandsdichte für den Wasserstoff in der Probe vorliegt. Das beobachtete Plateau zeigte sich allerdings nur in der ersten Beladung der Probe. Anscheinend reicht eine Entladespannung von 500mV nicht aus, um die Probe restlos zu entleeren, so dass weitere Beladungen schon "oberhalb" des Plateaus starteten.

In der linearen Auftragung ist deutlich zu erkennen, dass die Isothermen bei EMK-Werten von ca. $-150 \mathrm{mV}$ in ein zweites Plateau münden. Die Ursache dafür stellt die gleichzeitige Wasserstoff - Beladung der Pd-Deckschicht dar. Bei diesen EMK-Werten bildet sich im Pd ein Zwei-Phasen-Gebiet mit einer großen Zustandsdichte für den Wasserstoff aus. Es macht also keinen Sinn, über diesen Bereich hinaus zu messen, da die Konzentration in der amorphen Schicht jetzt nicht mehr genau bestimmt werden kann.

Die achsenvertauschten Messdaten $\mathrm{c}_{\mathrm{H}}(\mathrm{U})$ sind in der Abbildung 15 dargestellt

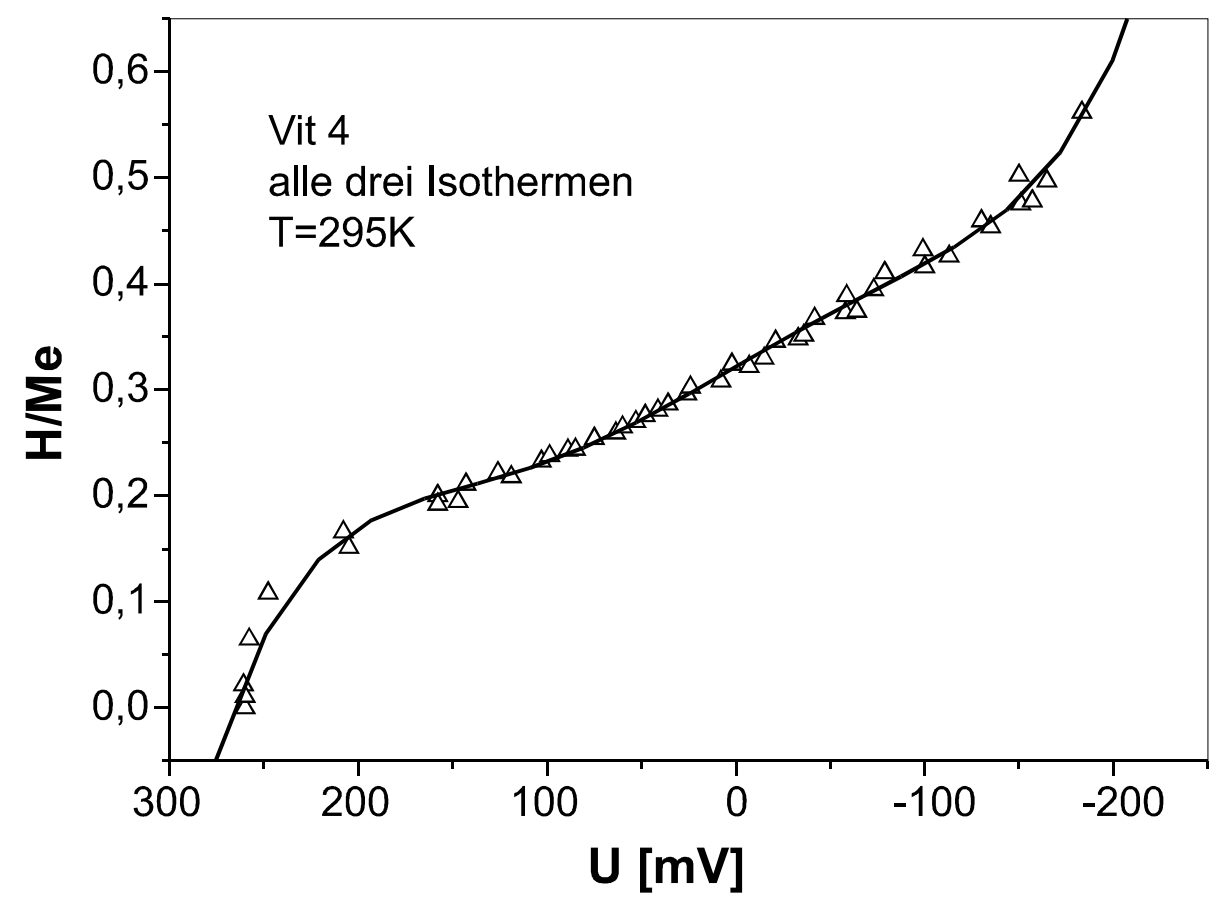

Abbildung 15: Isotherme an der Vit 4 - Probe. Die Auftragung ist hier im Vergleich zur linearen Auftragung in Abbildung 14 achsenvertauscht. 
Die durch Differentiation der Kurve erhaltene Zustandsdichte (s. Gleichung (4)) für die Vit 4 - Probe ist in Abbildung 16 zu sehen.

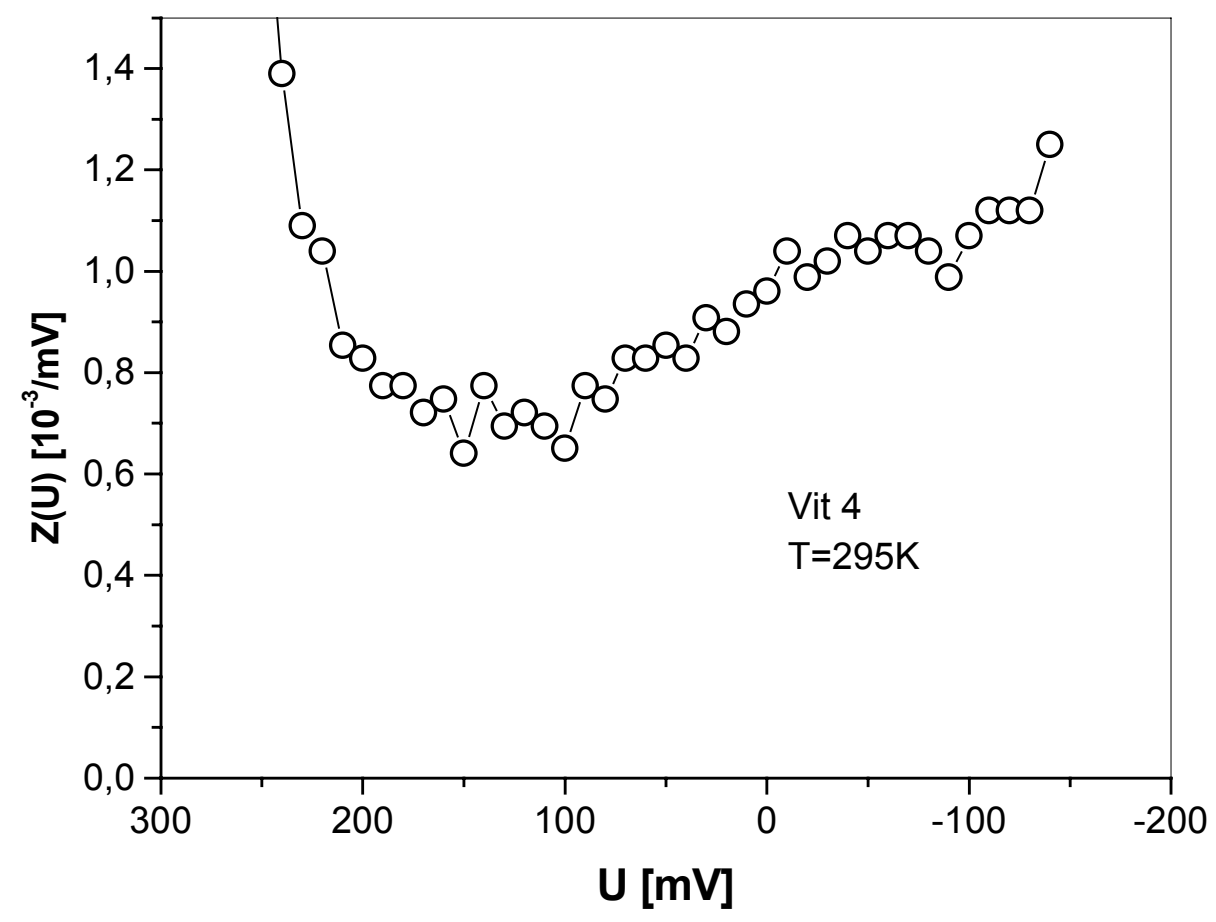

Abbildung 16: experimentell ermittelte Zustandsdichte an der Vit-4-Probe

Nach Umrechnung der in Abbildung 16 experimentell ermittelten Zustandsdichte Z(U) entsprechend der Gleichung (38), mit $U_{0}=-182 \mathrm{mV}$, ist diese Kurve mit der Platzenergieverteilung in der amorphen Probe bis auf den Strukturfaktor f (s. Kapitel 2.1.2) identisch.

Die Zustandsdichte zeigt ein asymmetrisches Minimum bei EMK $\approx 120 \mathrm{mV}$. Die steile Flanke liegt bei tiefen EMK-Werten. Zu größeren EMK-Werten hin steigt die Kurve relativ flach wieder an.

Analog zum vorigen Vorgehen wurde eine Isotherme an der Vitreloy 1 (Vit 1) - Probe bei Raumtemperatur aufgenommen und auf der folgenden Seite in Abbildung 17 dargestellt. 
a)

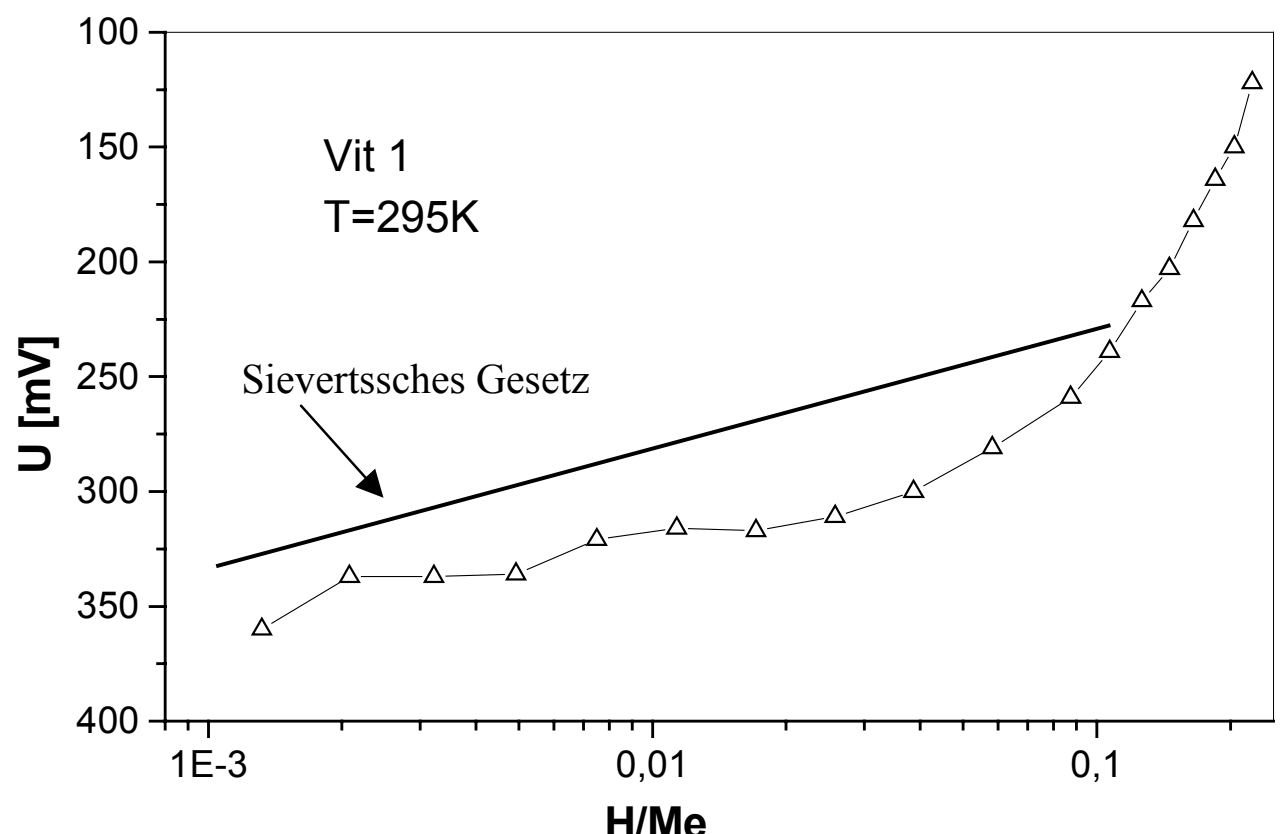

b)

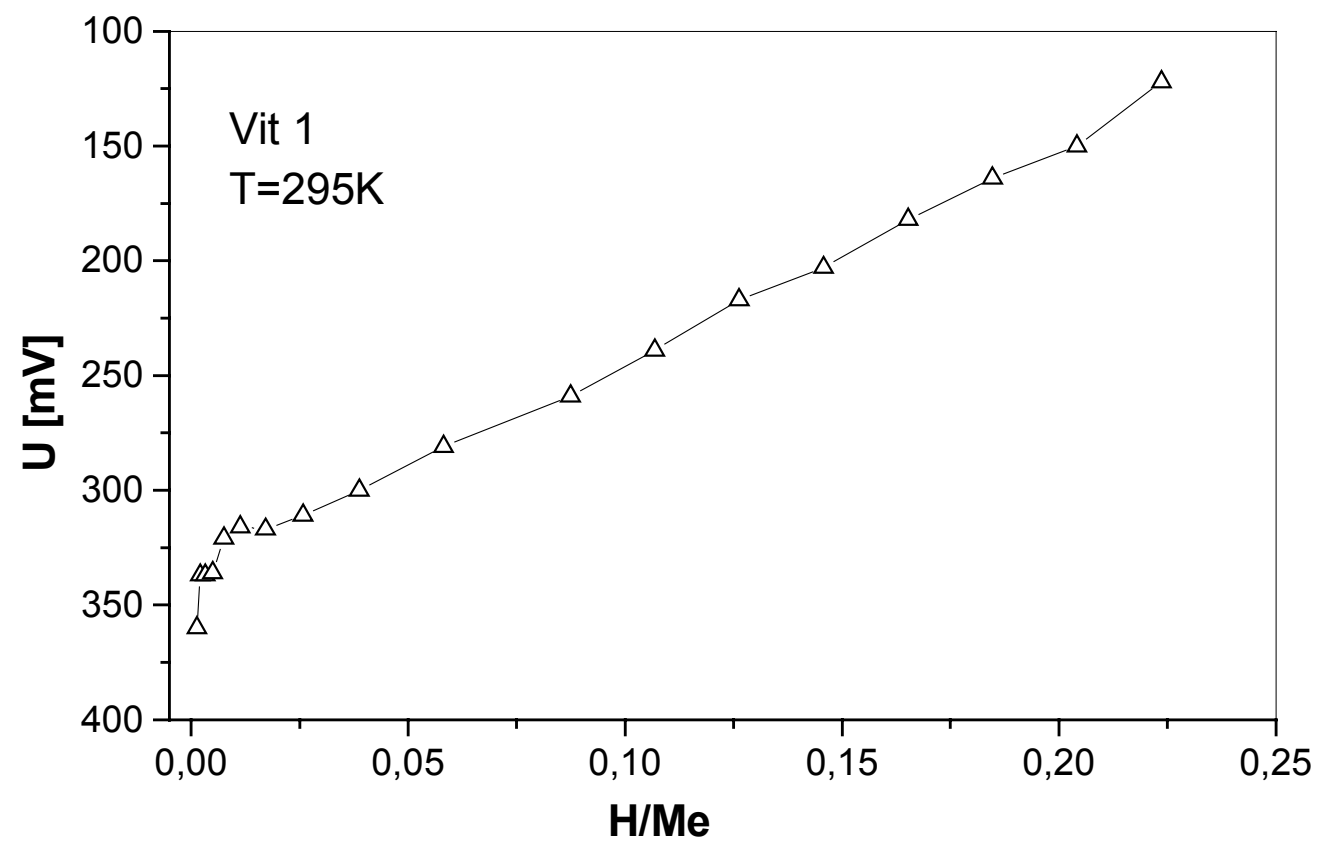

Abbildung 17: Isotherme an der amorphen $\mathrm{Zr}_{46.75} \mathrm{Ti}_{8.25} \mathrm{Cu}_{7.5} \mathrm{Ni}_{10} \mathrm{Be} e_{27.5}$. Die EMK wurde in a) gegen den Logarithmus der Konzentration aufgetragen. (Sieverts-Darstellung). Die in a) eingezeichnete Gerade hat die für eine ideal verdünnte Lösung erwartete Steigung. In Darstellung b) wurde die EMK linear gegen die Konzentration aufgetragen. 
Zusätzlich ist in Abbildung 17-a) eine Gerade mit der Sieverts-Steigung $\mathrm{m}_{\mathrm{S}}$ eingezeichnet. Vergleicht man die anfängliche Steigung der Isotherme mit der Steigung $\mathrm{m}_{\mathrm{S}}$, so stellt man fest, dass die Probe für Konzentrationen kleiner als $c_{H}=0,10 \mathrm{H} / \mathrm{Me}$ nahezu ein Sievert-Verhalten zeigt. Da das Sievert-Verhalten für ideale verdünnte Lösungen gültig ist, ist dies ist ein Hinweis darauf, dass die Ausgangskonzentration in dieser Probe sehr gering war. Darauf deutet auch hin, dass die Isotherme sehr tief verläuft. An dieser Probe konnte nur eine Messung aufgenommen werden, da bei der Entladung mit einer Spannung von $800 \mathrm{mV}$ die $\mathrm{Pd}-$ Deckschicht und sehr wahrscheinlich auch die amorphe Legierung oxidierte. Die Probe war danach für weitere Beladungsexperimente unbrauchbar.

Abbildung 18 und Abbildung 19 zeigen die achsenvertauschte Darstellung der Isotherme, bzw. die daraus ermittelte Zustandsdichte.

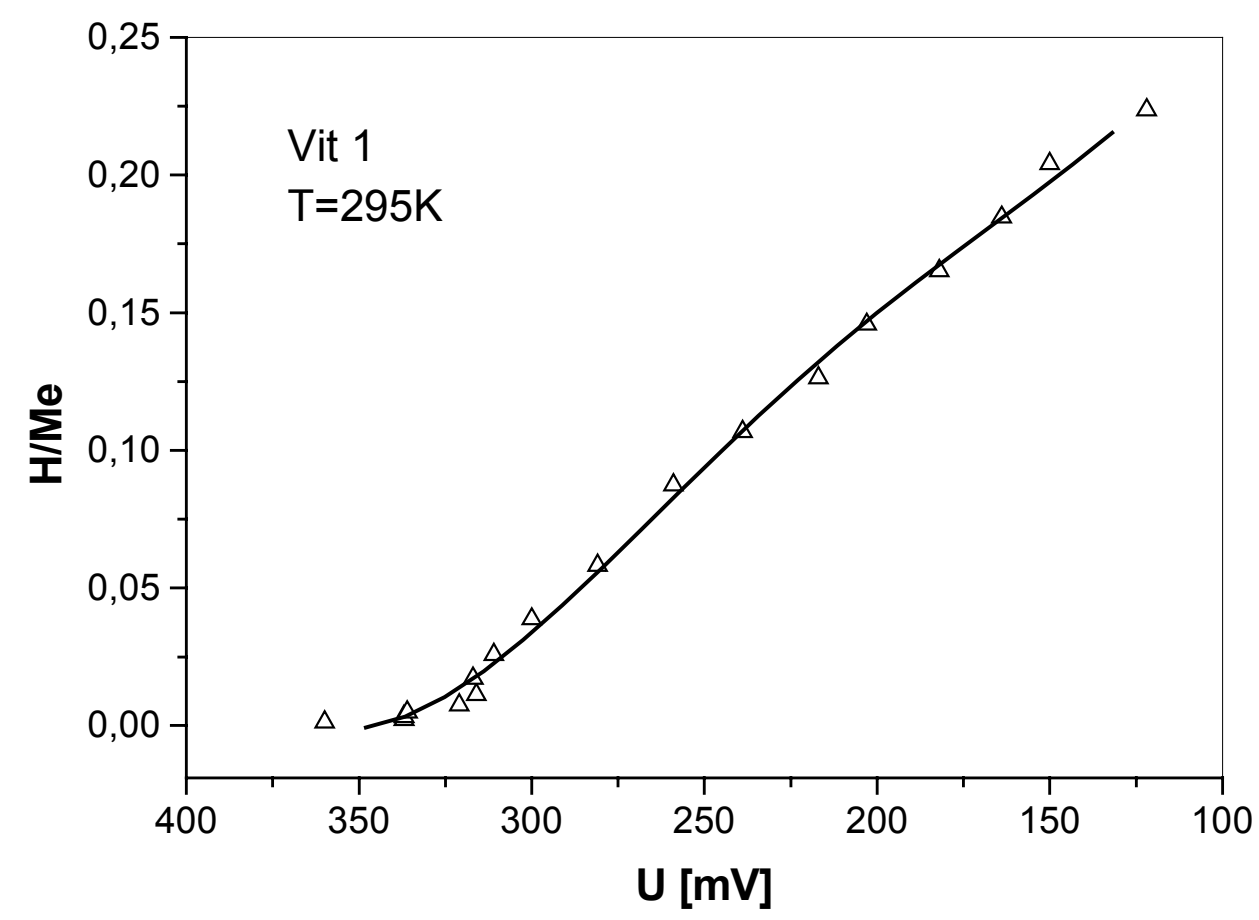

Abbildung 18: Isotherme an der amorphen $\mathrm{Zr}_{41} \mathrm{Ti}_{14} \mathrm{Cu}_{12.5} \mathrm{Ni}_{10} \mathrm{Be} e_{22.5}$-Probe Auftragung Konzentration $\mathrm{c}_{H}$ gegen $U\left(\right.$ statt $U\left(c_{H}\right)$ !) 


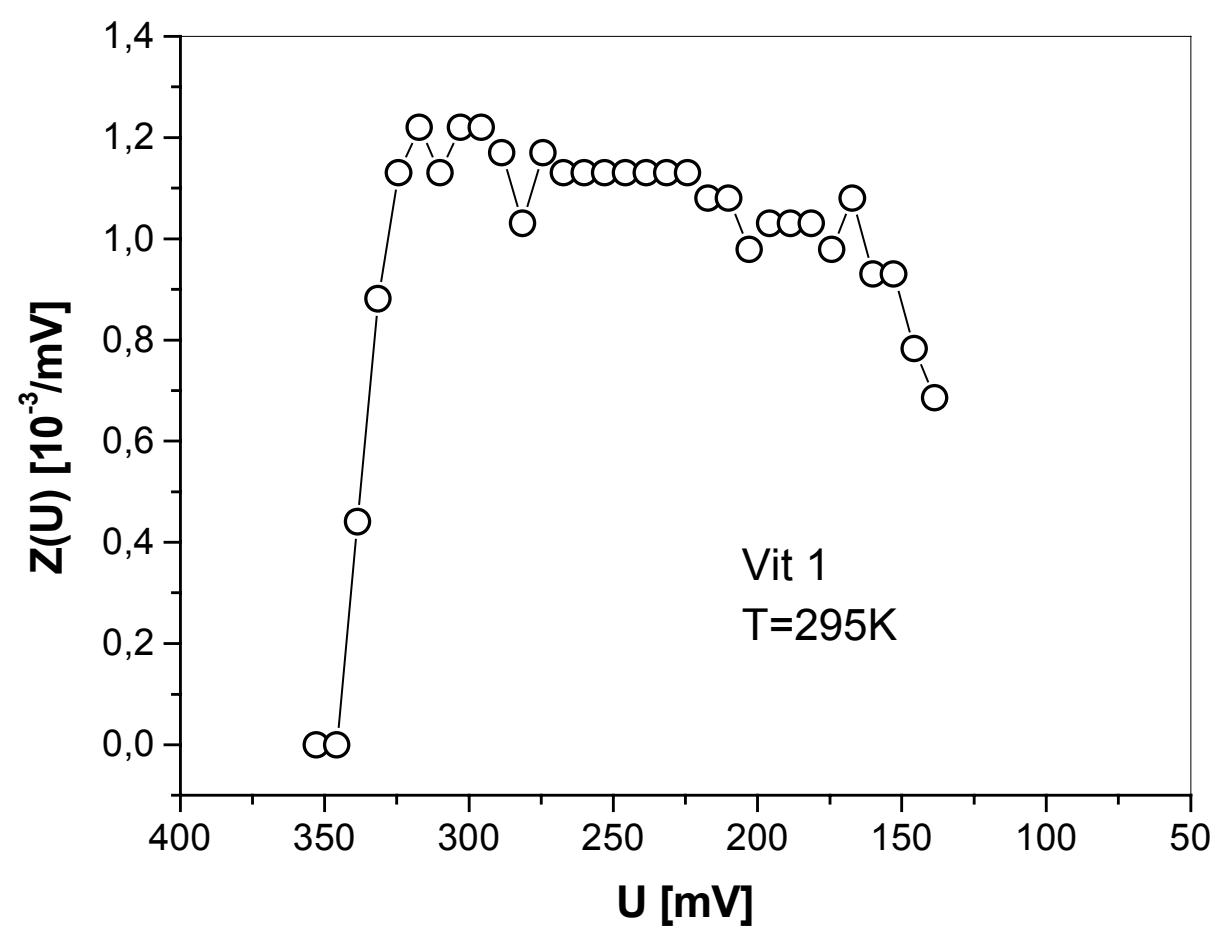

Abbildung 19: Experimentell ermittelte Zustandsdichte an der Vit - 1-Probe

In der im Vit 1 bestimmten Zustandsdichte ist zunächst ein steiler Anstieg zu beobachten, dem sich ein Maximum bei ca. $300 \mathrm{mV}$ anschließt. Zu höheren EMK-Werten nimmt die $\mathrm{Z}(\mathrm{U})$ stetig und relativ flach wieder $a b$.

\subsubsection{ZANC-Legierung}

Im Gegensatz zu den Isothermen an Vit 1 und Vit 4 wurde die Messungen der Isothermen an den amorphen $\mathrm{Zr}_{66,8} \mathrm{Al}_{17,4} \mathrm{Ni}_{7,2} \mathrm{Cu}_{8,8}$-Proben (ZANC) mit der in Kapitel 3.2.1 beschriebenen automatischen Zeitschaltuhr gemessen. In Abbildung 20 ist diese Form der Beladung dargestellt. Es sind die EMK-Gleichgewichtseinstellungen gegen die Zeit aufgetragen. Nach jedem Beladungspuls nimmt die EMK exponentiell ab, jedoch sieht man in dem unteren vergrößerten Ausschnitt, dass sich keine Gleichgewichtswerte in der vorgegebenen Zeit einstellen. Für die weitere Auswertung sei darauf hingewiesen, dass nach der ca. 35. bis 
40. Beladung die Abklingkurven irreguläre Verläufe zeigten. Dies zeigt der obere Vergrößerungsausschnitt in Abbildung 20. Höchstwahrscheinlich handelt es sich dabei um schwer bestimmbare chemische Reaktionen an der Probenoberfläche, weshalb die Aussagekraft der Messwerte ab diesem Bereich nicht besonders groß ist.

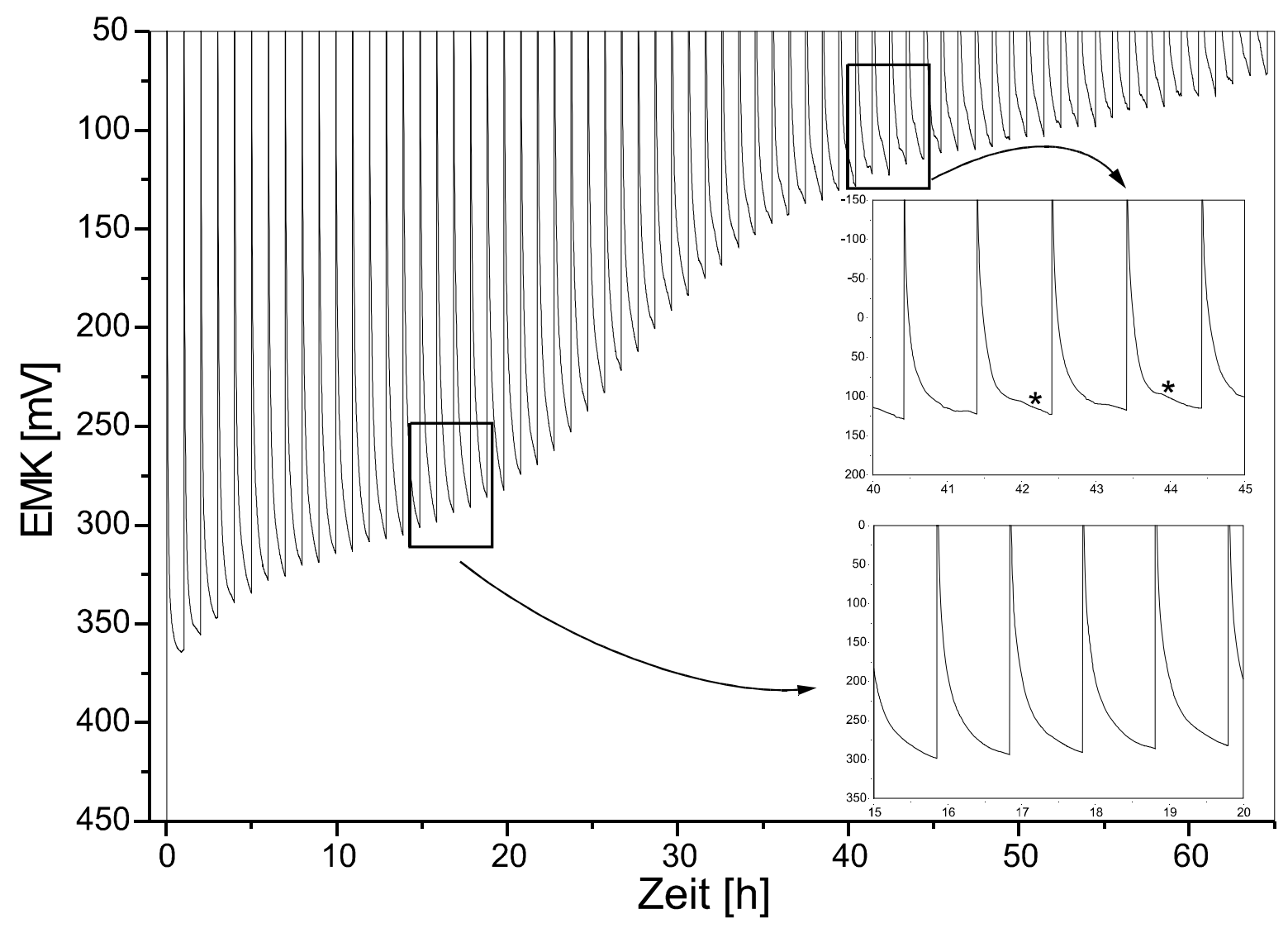

Abbildung 20: Beladungsexperiment an der $\mathrm{Zr}_{66,8} \mathrm{Al}_{17,4} \mathrm{Ni}_{7,2} \mathrm{Cu}_{8,8}$ - Probe ZANC-6a.

Des weiteren erkennt man in Abbildung 20, dass sich der Endwert der EMK, ermittelt nach jeweils 60 Minuten, verändert. Trägt man diese Endpunkte gegen die gesamte Wasserstoffkonzentration in der Probe auf, so erhält man die in Abbildung 22 gezeigte (Nicht-Gleichgewichts-)Isothermen (helle Kreise). Zusätzlich eingetragen ist die analog ermittelte Isotherme einer zweiten Probe (dunkle Kreise), die eine sehr gute Reproduzierbarkeit der gewonnenen Messdaten belegt. Die dritte Isotherme in Abbildung 22 (Sterne) wurde erhalten, als sich zwischen zwei Beladungsschritten eine Gleichgewichts-EMK einstellen konnte. Letztere wurde ohne Zeitschaltuhr an einer dritten, aber mit den beiden Proben vorher identischen Probe, bestimmt. In allen drei Kurven deutet sich ein Plateau bei $\mathrm{c}_{\mathrm{H}} \approx 0,15 \mathrm{H} / \mathrm{Me}$ an. Bei Konzentrationen bis $\mathrm{c}_{\mathrm{H}} \approx 0,40 \mathrm{H} / \mathrm{Me}$ zeigen alle 
drei Isothermen einen vergleichbaren Verlauf, mit dem Unterschied, dass die Nicht-Gleichgewichts - Isothermen etwas oberhalb der dritten Isotherme liegen. $\mathrm{Ab}$ $\mathrm{c}_{\mathrm{H}}=0,40 \mathrm{H} / \mathrm{Me}$ knicken die beiden Nicht - Gleichgewichts - Isothermen ab und scheinen ein zweites Plateau auszubilden. Es kann sich hier noch nicht um die Bildung des PalladiumHydrides in der Deckschicht handeln, da das dabei zu erwartende PdH - Plateau bei ca. $-130 \mathrm{mV}$ liegt. Die Ausbildung dieses zweiten Plateaus ist in der Gleichgewichtsisotherme nicht zu sehen. Die folgende Auswertung ignoriert dieses zweite Plateau, da dort die bereits erwähnten Irregularitäten in den Abklingkurven beobachtet worden sind.

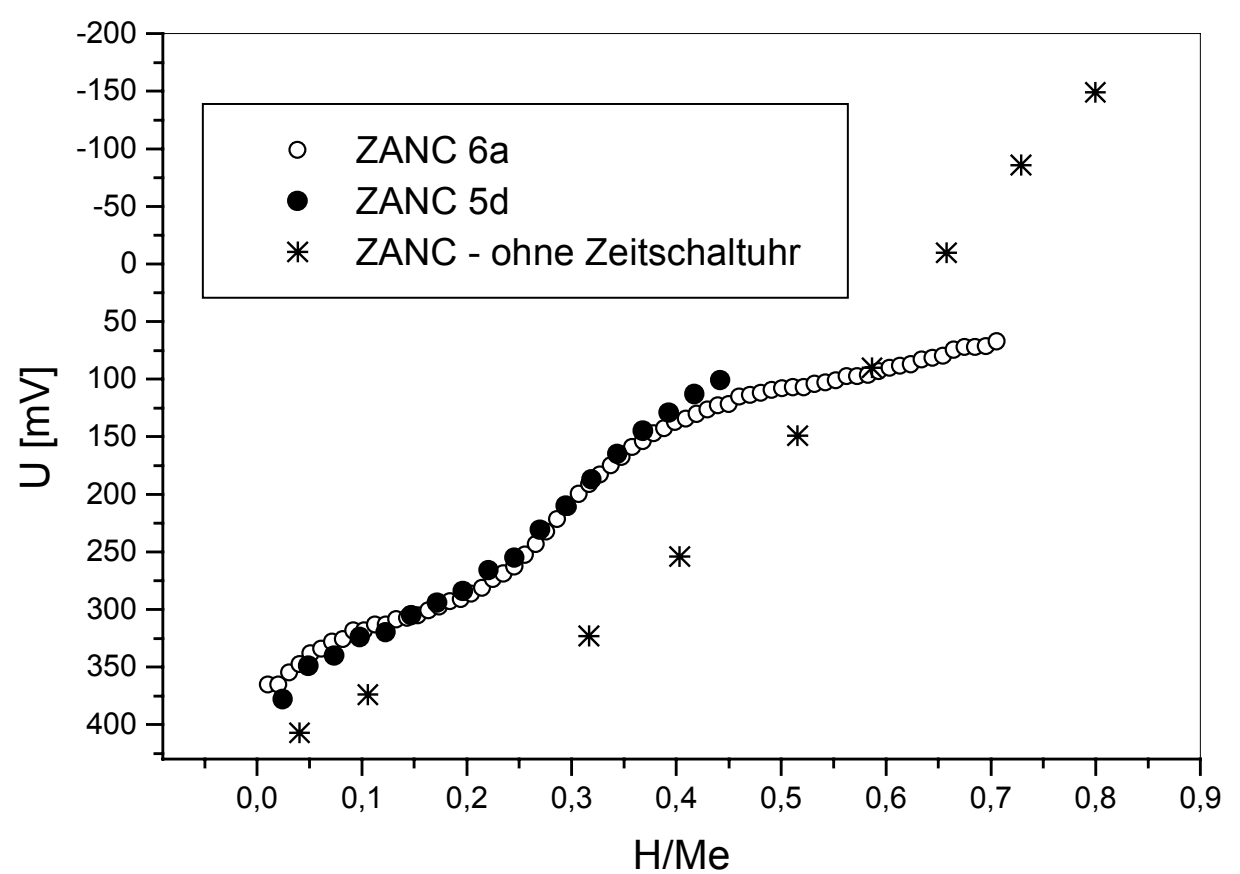

Abbildung 21: Gemessene Isothermen an den Proben ZANC-6a und ZANC-5d (mit Zeitschaltuhr gemessenNicht-Gleichgewichts-Isotherme) und eine Isotherme an der ZANC-1--Probe (ohne Zeitschaltuhr gemessen - Gleichgewichtsisotherme)

Die erhaltenen Zustandsdichten sind in Abbildung 22 für alle drei Isothermen zu sehen. Es wurde bereits die Umrechnung von $Z(U)$ zu Z(E) durchgeführt. Alle drei Z(E) zeigen bei $\mathrm{E} \approx 50 \mathrm{~kJ} / \mathrm{mol}$ bis $55 \mathrm{~kJ} / \mathrm{mol}$ ein Maximum. Die Maxima aus den Nicht - Gleichgewichts Isothermen sind schmaler als das Maximum in der Zustandsdichte der dritten Isotherme. 


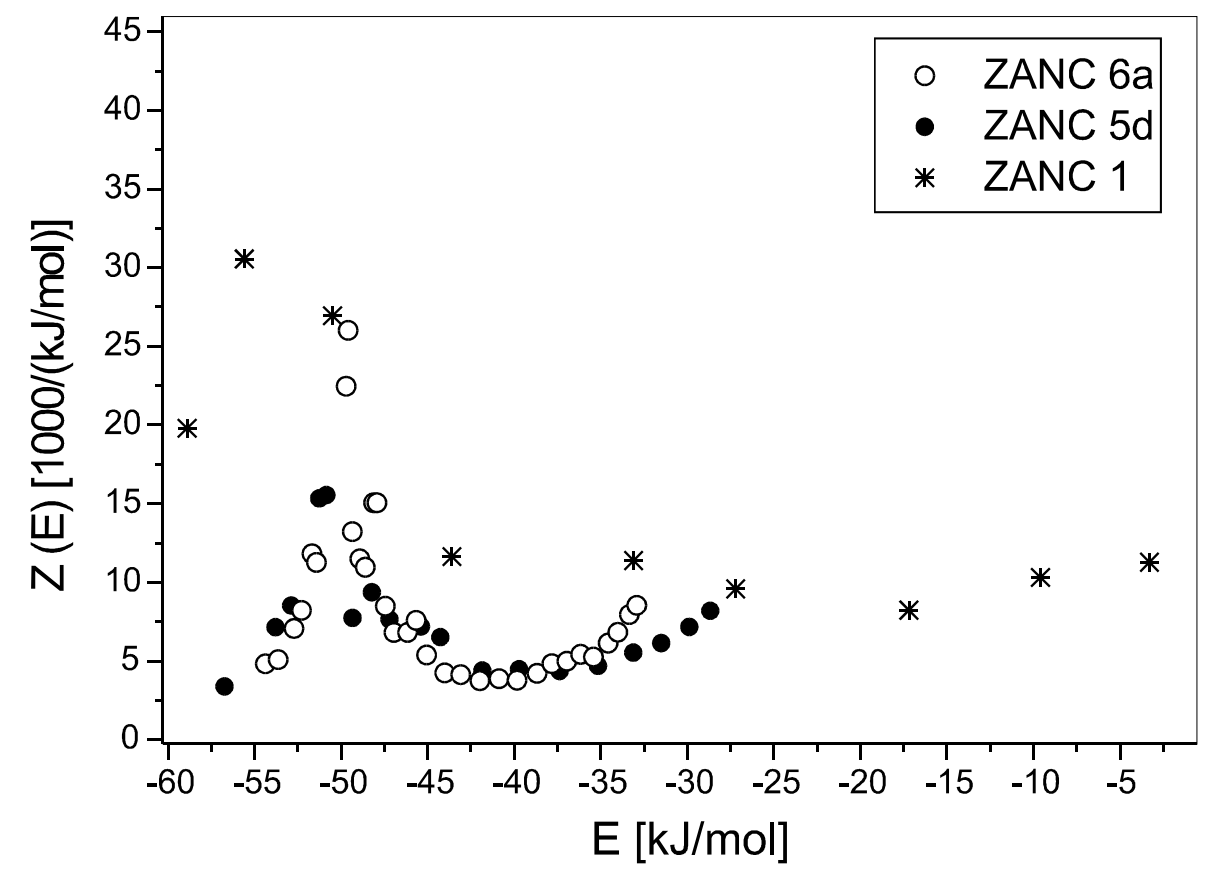

Abbildung 22: Z(E) aus Nicht-Gleichgewichts-Isothermen an den Proben ZANC-6a und ZANC-5d (helle und dunkle Kreise) und Z(E) aus der Gleichgewichtsisotherme an der Probe ZANC-1. 


\subsection{Modellierte Zustandsdichten Z(E)}

Im Kapitel 2.3 wurde eine Methode vorgestellt, die Zustandsdichten Z(E) mehrkomponentiger amorpher Legierungen zu berechnen. Dabei berücksichtigt der Parameter $\sigma$ die Breite der Verteilung der Platzenergien eines jeden Tetraedertyps. Des weiteren wurde ein zusätzlicher Verzerrungsbeitrag zu den Platzenergien eingeführt, der dem Volumenunterschied eines Tetraeders in der Legierung im Vergleich zu einem freien Tetraeder Rechnung trägt. Dieser Beitrag wird durch einen Laufparameter S berücksichtigt. Es wird gezeigt, welchen Einfluss diese Parameter auf die berechneten Zustandsdichten haben. Dazu wird angenommen, dass $\sigma$ und $S$ für alle Tetraeder gleich sind. Die Berechnungen werden sowohl für Vitreloy als auch für die ZANC-Legierung vorgestellt. Beide amorphe Systeme unterscheiden sich jedoch nicht nur in der Anzahl der Komponenten. In Vitreloy ist der Unterschied zwischen dem größten Tetraedervolumen des $\mathrm{Zr}_{4}$-Teraeders und dem kleinsten, dem $\mathrm{Be}_{4}$-Tetraeder besonders groß. Hier erwartet man besonders große Veränderung durch die Berücksichtigung der Verzerrungsenergie.

Alle die zur Berechnung der Zustandsdichten notwendigen Parameter sind in der Tabelle 4 aufgelistet:

Tabelle 4: verwendete Parameter zur Berechnung von Z(E) für Vitreloy und ZANC

\begin{tabular}{|c|c|c|c|c|c|c|}
\hline Element & $\%$ in Vit-4 & $\%$ in Vit-1 & \% in ZANC & $\begin{array}{l}\mathbf{E}_{i}^{\mathbf{0}_{i}^{(1)}} \\
{[\mathrm{kJ} / \mathbf{m o l ~ H}]}\end{array}$ & $\begin{array}{l}\mathbf{V}_{\text {mol }}{ }^{(2)} \\
{\left[\mathbf{c m}^{3}\right]}\end{array}$ & $\begin{array}{l}\mathbf{R}_{\text {atdom }}{ }^{(3)} \\
\text { [nm] }\end{array}$ \\
\hline Zirkonium & 46,75 & 41,00 & 66,8 & $-59,52$ & 14,02 & 0,155 \\
\hline Titan & 8,25 & 14,00 & - & $-45,24$ & 10,64 & 0,140 \\
\hline Kupfer & 7,50 & 12,50 & 8,8 & $+49,04$ & 7,11 & 0,135 \\
\hline Nickel & 10,00 & 10,00 & 7,2 & $+16,68$ & 6,59 & 0,135 \\
\hline Beryllium & 27,50 & 22,50 & - & $-1,84$ & 4,85 & 0,105 \\
\hline Aluminium & - & - & 17,4 & $+63,20$ & 10,00 & 0,125 \\
\hline
\end{tabular}


Zur Berechnung der elastischen Energiebeiträge nach Gleichung (32) wurde laut Literaturangaben davon ausgegangen, dass das Volumen eines Wasserstoffatoms $\mathrm{V}_{\mathrm{K}}=2,8 \AA^{3}$ beträgt [Fuk93]. Für den Schermodul wurde der Wert von $\mu=35,0 \mathrm{GPa}$ [Flo01] eingesetzt.

\subsubsection{Vitreloy-Legierungen}

Die Vitreloy Legierungen bestehen aus 5 Elementen, daher sind nach Gleichung (16) 70 verschiedene Tetraeder bei der Berechnung der Zustandsdichte zu berücksichtigen. Im Anhang sind diese 70 Tetraeder benannt und ihre Energien sowohl mit als auch Volumenkorrektur zu finden. Als Auszug sind in der folgenden Tabelle die Tetraeder der reinen Elemente aufgelistet, um im folgenden am Beispiel von Vit-4 den wesentlichen Einfluss der elastischen Energie zu demonstrieren (Für den Laufparameter S in Gleichung (30) wurde der Wert von 0,5 eingesetzt):

$\begin{aligned} & \text { Tabelle 5: berechnete Energien der fünf, ,reinen “ Tetraeder in Vit - } 4 \\
& \text { Tetraeder }\end{aligned}$
\begin{tabular}{l|l|l}
$\mathbf{0 h n e} \Delta \mathbf{E}_{\mathbf{e l}}$ & $\mathbf{m i t} \Delta \mathbf{E}_{\mathbf{e l}}$ \\
\hline $\mathrm{Zr} 4$ & $-59,52$ & 16,38 \\
$\mathrm{Ti} 4$ & $-45,24$ & $-25,23$ \\
$\mathrm{Be} 4$ & $-1,84$ & $-503,19$ \\
$\mathrm{Ni4}$ & 16,68 & $-122,19$ \\
$\mathrm{Cu} 4$ & 49,04 & $-64,88$
\end{tabular}

Die Berücksichtigung der elastischen Energiebeiträge führt zu einer starken Verschiebung der Lösungsenergie für den Wasserstoff. Die berechneten Energien sind teilweise jedoch so groß, dass sie gar keine Lösungs- sondern vielmehr kovalente Bindungsenergien darstellen. Besonders im Fall des reinen Berylliumtetraeders, dem kleinsten aller Elemente in der Legierung, wird dieser Effekt deutlich: Eine Energie von $-500 \mathrm{~kJ} / \mathrm{mol}$ liegt bereits weit im Bereich kovalenter Bindungen. So ist die Bindungsenthalpie zum Beispiel zwischen den 
beiden $\mathrm{H}$-Atomen im $\mathrm{H}_{2}$-Molekül ca. $-430 \mathrm{~kJ} / \mathrm{mol}$, die zwischen Wasserstoff und Beryllium im Berylliumhydrid BeH ca. $-200 \mathrm{~kJ} / \mathrm{mol}$. Man stellt weiter fest, dass Zirkonium, das Element mit der stärksten Wasserstoffbindung, jetzt den Tetraeder bildet, der von den vier reinen Tetraedern am wenigsten attraktiv ist. In Abbildung 23 sind die Energieverteilungen von Vit 4 sowohl mit als auch ohne den Beitrag der elastischen Energien dargestellt. Für eine bessere Übersicht sind hier die nach Gleichung (33) berechneten Lagen der mittleren Energien der Tetraeder dargestellt. Die Höhe der Peaks entspricht der nach Gleichung (20) berechneten Wahrscheinlichkeit $\mathrm{W}_{\mathrm{i}}$. Der Wasserstoffblocking-Effekt (s. Kapitel 2.3.3) wurde hier bereits berücksichtigt.

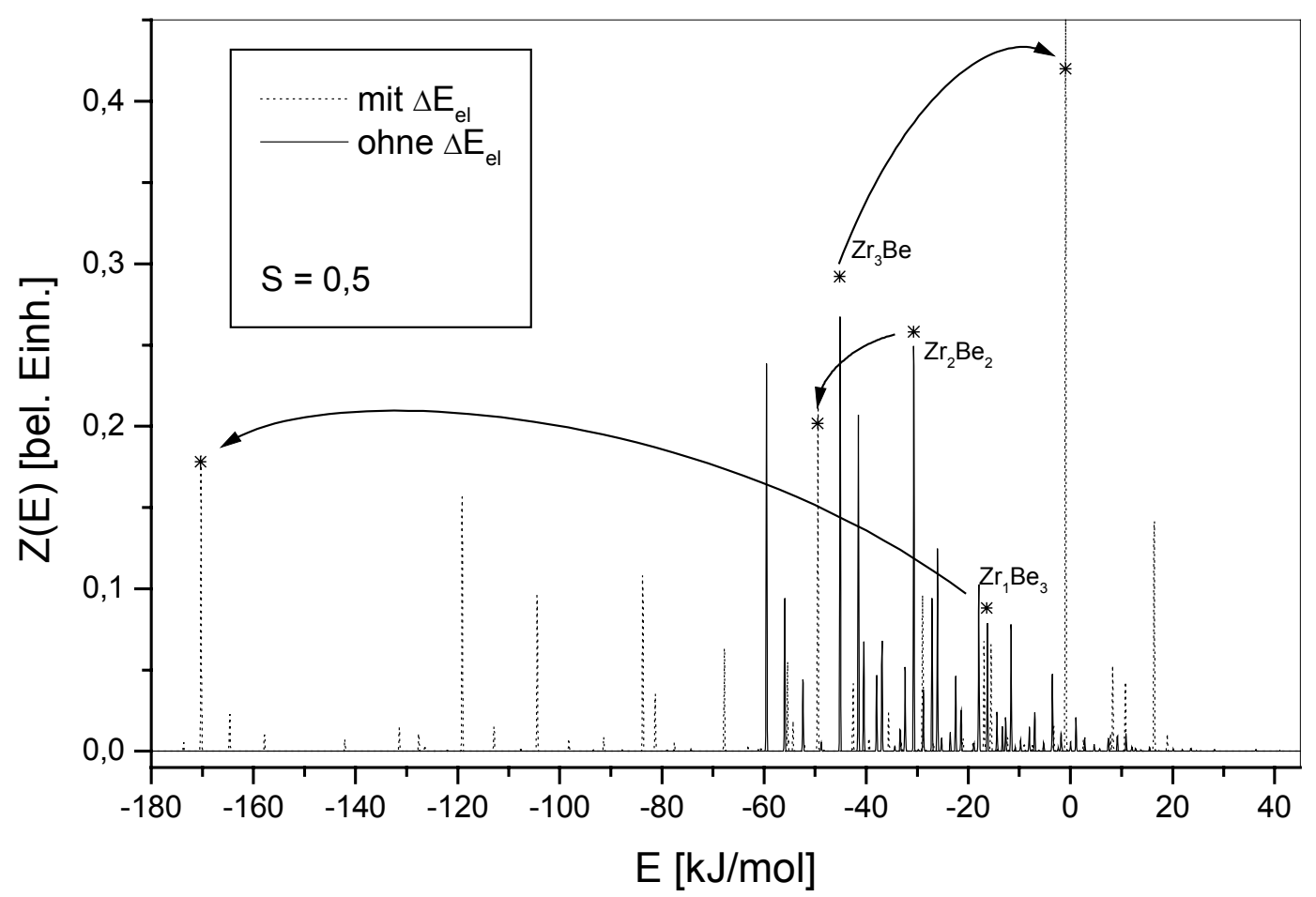

Abbildung 23: Energieverteilungen der verschiedenen 70 Tetraeder in Vit 4 mit und ohne Berücksichtigung der elastischen Energiebeiträge.

Die unterschiedliche Verschiebung der Platzenergien für verschiedene Tetraeder ist in dieser Darstellung zu erkennen. Für die drei Tetraeder Zr3Be, Zr2Be2 und ZrBe3 ist dies durch die eingezeichneten Pfeile hervorgehoben worden So steigt die Platzenergie des $\mathrm{Zr}_{3} \mathrm{Be}_{1}$-Teteraeders von $-45,10 \mathrm{~kJ} / \mathrm{mol}$ ohne $\Delta \mathrm{E}_{\mathrm{el}}$ auf $-0,96 \mathrm{~kJ} / \mathrm{mol}$ mit $\Delta \mathrm{E}_{\mathrm{el}}$ an. Der Tetraeder $\mathrm{Zr}_{1} \mathrm{Be}_{3}$ dagegen verschiebt sich in der Platzenergie von $-16,26 \mathrm{~kJ} / \mathrm{mol}$ auf 
$-170,25 \mathrm{~kJ} / \mathrm{mol}$. Neben dieser Verschiebung wird in Abbildung 23 die durch die Wasserstoffblocking bedingte Veränderung der $\mathrm{W}_{\mathrm{i}}$, bei Berücksichtigung des elastischen Energiebeitrags, deutlich.

Um den Einfluss des Parameters S auf die berechneten Zustandsdichten zu demonstrieren, wurden verschiedene $Z(E)$ berechnet, in denen $S$ von $S=0,40$ bis $S=0,65$ verändert wurde. Für alle berechneten Verläufe wurde $\sigma=10 \mathrm{~kJ} / \mathrm{mol}$ verwendet. Die sich ergebenen verschiedene Verläufe für Vit 4 sind in Abbildung 24 dargestellt.

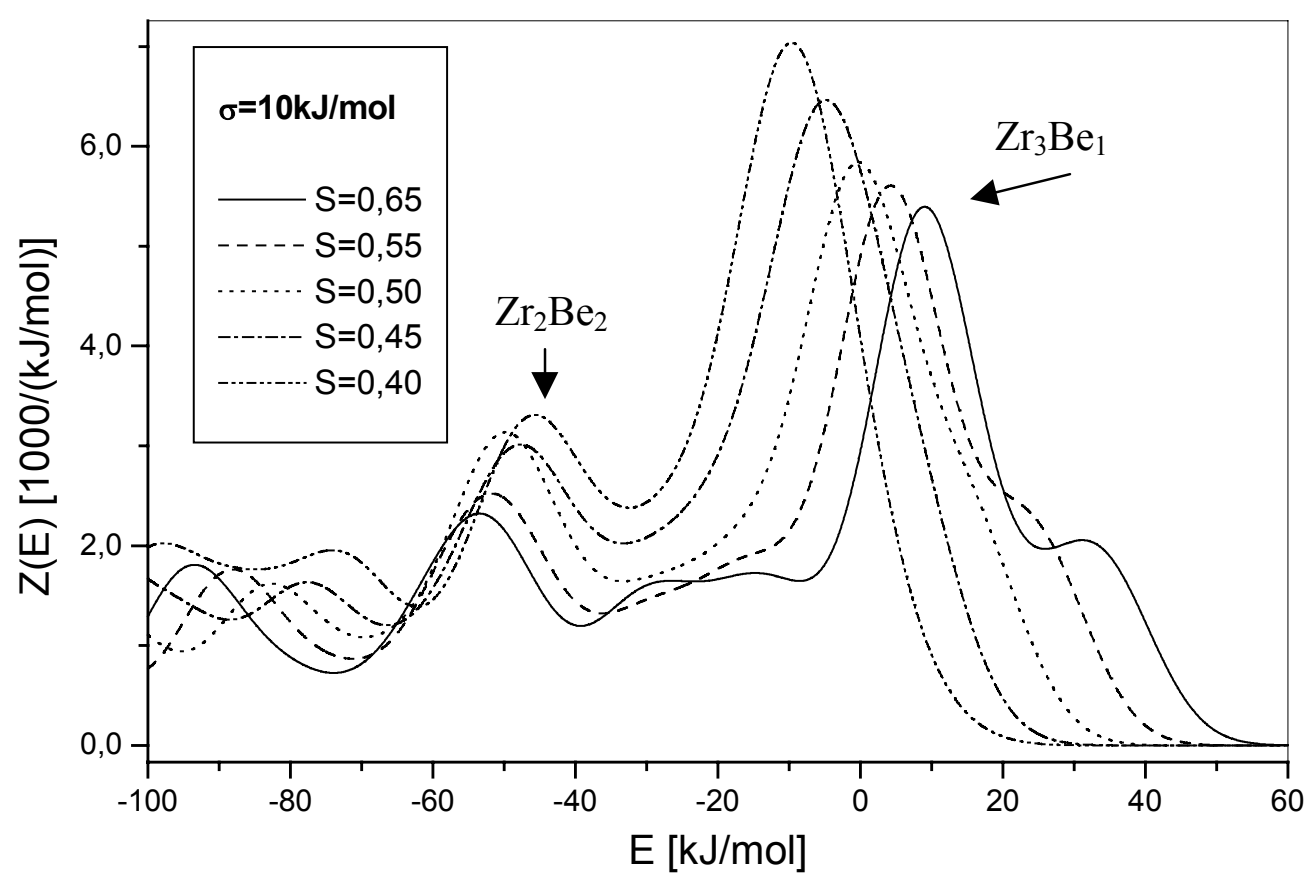

Abbildung 24: Einfluss des Laufparameters S auf die Berechneten Zustandsdichte von Vit 4

Dargestellt wurde der Übersichtlichkeit halber nur der Ausschnitt der Zustandsdichten von $\mathrm{E}=-100 \mathrm{~kJ} / \mathrm{mol}$ bis $\mathrm{E}=+60 \mathrm{~kJ} / \mathrm{mol}$. Die beiden größten Maxima korrespondieren $\mathrm{zu}$ den statistisch am häufigsten Tetraedertypen $\mathrm{Zr}_{3} \mathrm{Be}_{1}$ und $\mathrm{Zr}_{2} \mathrm{Be}_{2}$ (s. Anhang). Zwischen diesen Maxima liegt ein durch asymmetrische Flanken begrenztes Minimum in der Zustandsdichte, dass sich mit zunehmenden $\mathrm{S}$ - Parameter verbreitert. Bei hohen $\mathrm{S}$ - Werten bilden sich zusätzliche Strukturen im Minimum aus. Die Wahl des S-Parameters verändert also die Lage, den Abstand und die Höhe der Zustandsmaxima. 
In der Abbildung 25 wurde der Laufparameter $\mathrm{S}$ konstant bei $\mathrm{S}=0,45$ gehalten und die Breite der Verteilungen $\sigma$ zwischen $\sigma=6 \mathrm{~kJ} / \mathrm{mol}$ und $\sigma=14 \mathrm{~kJ} / \mathrm{mol}$ variiert. Da es sich um die gleiche berechnete Legierung Vit 4 handelt, sind die Kurven mit $\sigma=10 \mathrm{~kJ} / \mathrm{mol}$ und $\mathrm{S}=0,50$ in Abbildung 24 und Abbildung 25 identisch. Die Wahl von $\sigma$ bestimmt also im wesentlichen die Höhe der Zustandsdichtemaxima. Große Werte von $\sigma$ führen dazu, dass kleine Nebenmaxima nicht mehr aufgelöst werden können.

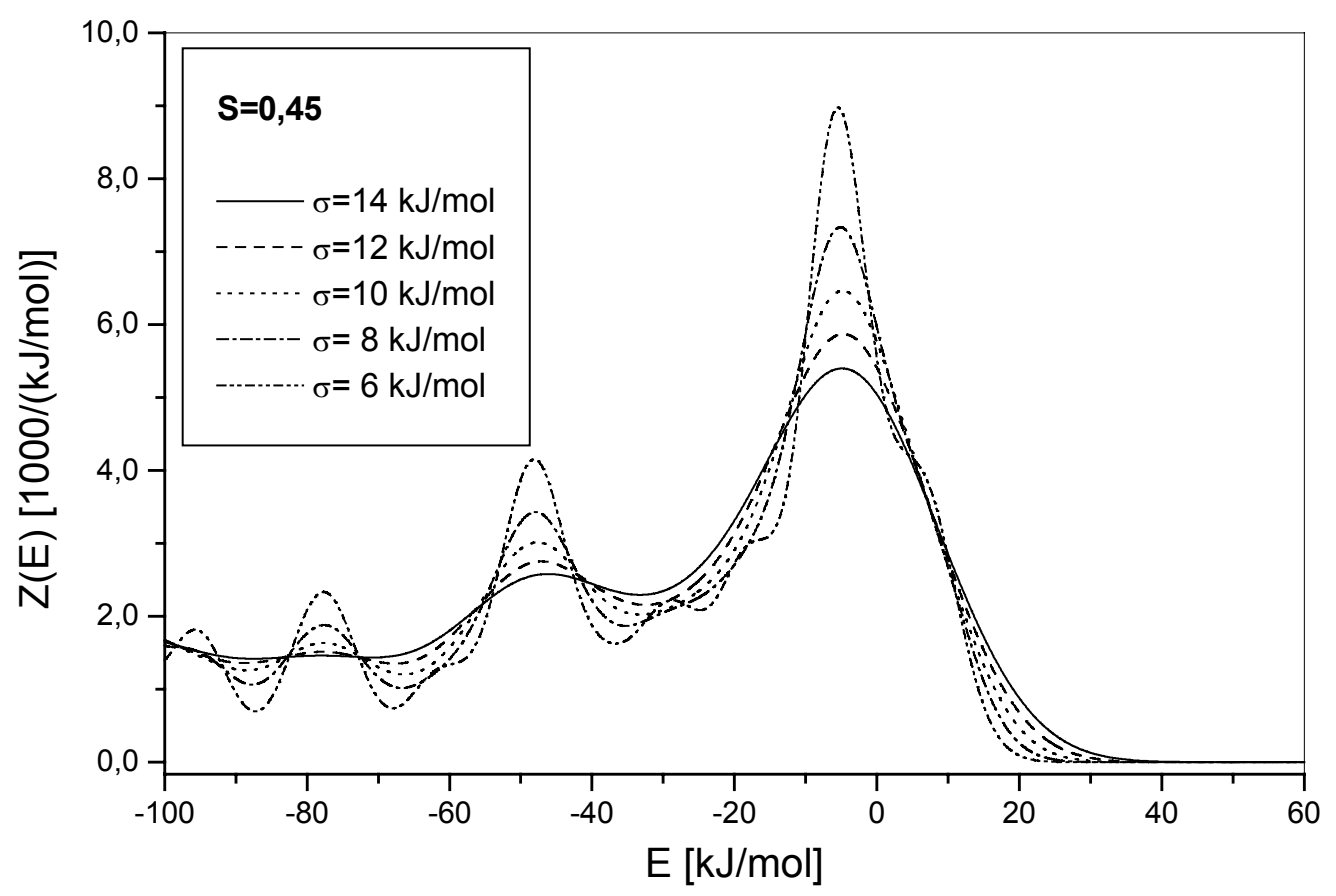

Abbildung 25: Einfluss der Halbwertsbreite s der einzelnen Verteilungen auf die Zustandsdichte von Vit 4

Trotz gleicher Komponenten der Legierungen Vit 4 und Vit 1 ergibt sich nach dem Modell aufgrund der anderen Konzentrationen eine unterschiedliche Zustandsdichte für die beiden Legierungen. In der Abbildung 26 sind die berechneten Zustandsdichten sowohl für Vit 1 als auch für Vit 4 in einem größerem Energieintervall gezeigt: 


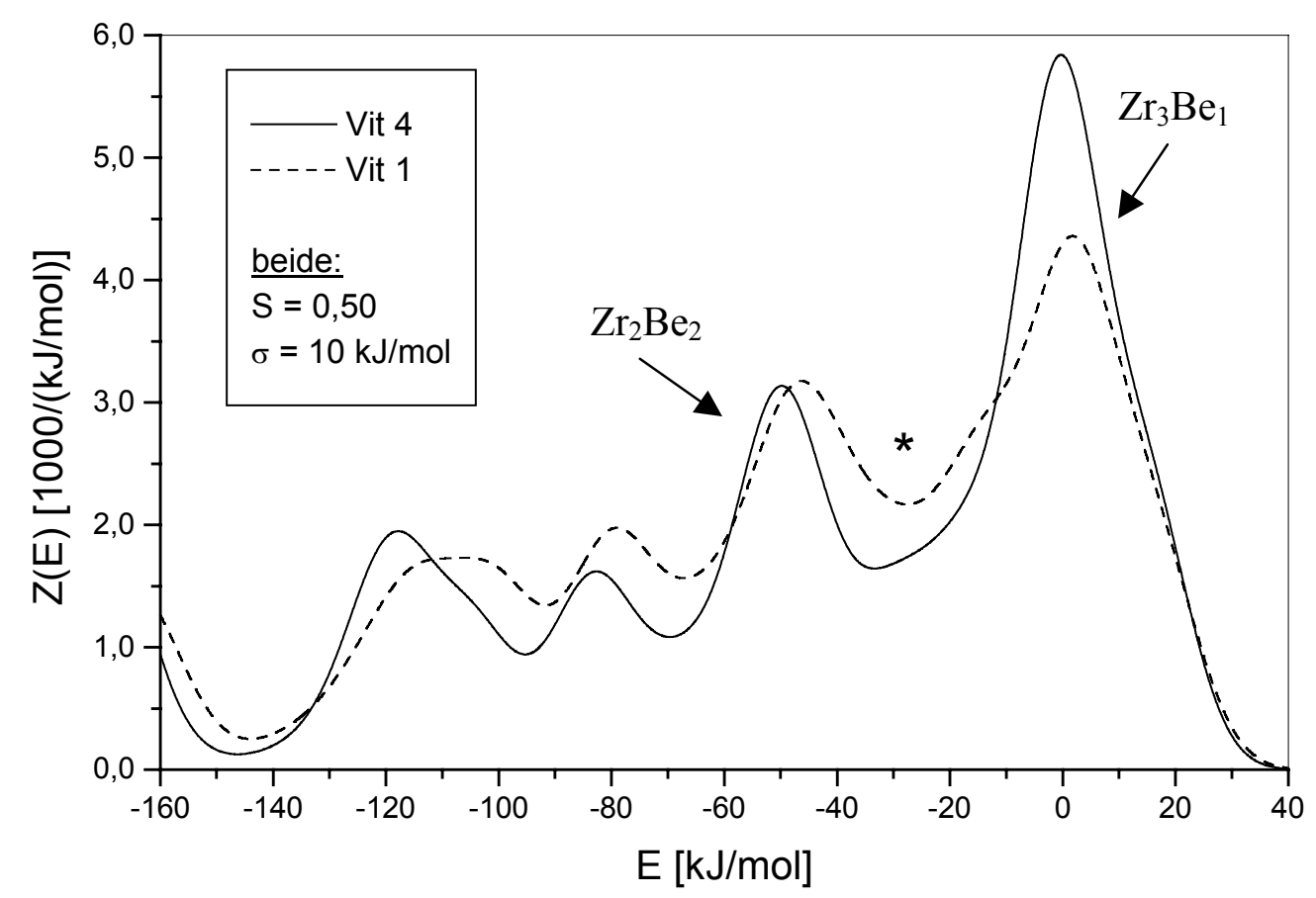

Abbildung 26: Vergleich der berechneten Zustandsdichten für die beiden Legierungen Vit 4 und Vit 1

Zur Berechnung beider Kurven in Abbildung 26 wurde der Parameter $\mathrm{S}=0,50$ und die Halbwertsbreite der einzelnen Tetraederverteilungen mit $\sigma=10 \mathrm{~kJ} / \mathrm{mol}$ eingesetzt. Der in der Grafik eingezeichnete Stern kennzeichnet das im vorangegangenen Abschnitt beschriebene Minimum. Deutlich ist die Abnahme des großen $\mathrm{Zr}_{3} \mathrm{Be}_{1}-\mathrm{Maximums}$ bei ca. $\mathrm{E}=0 \mathrm{~kJ} / \mathrm{mol} \mathrm{zu}$ erkennen, die natürlich aus der im Vit 1 geringeren Konzentration an Zirkonium herrührt $\left(\mathrm{c}_{\mathrm{Zr}, \mathrm{Vit1}}=0,41 ; \mathrm{c}_{\mathrm{Zr}, \mathrm{Vit}}=0,468\right)$. Die Effekte durch die Variation der Parameter $S$ und $\sigma$ sind qualitativ identisch mit denen von Vit 4. Bei gleichem Laufparameter $\mathrm{S}$ sind jedoch unterschiedliche Positionen der einzelnen Maxima $\mathrm{zu}$ beobachten. Dies ist ein Effekt der Berechnung der elastischen Energiebeiträge, in denen das nach Gleichung (27) berechnete Molvolumen ein Maß für den zusätzlichen Energiebeitrag ist. Die berechneten Molvolumina der beiden Legierungen sind aufgrund der verschiedenen Konzentrationen der einzelnen Komponenten in der Legierung unterschiedlich. 


\subsubsection{ZANC-Legierung}

Die ZANC Legierung besteht aus 4 Elementen, daher sind nach Gleichung (16) 35 verschiedene Tetraeder bei der Berechnung der Zustandsdichte zu berücksichtigen. Eine Liste dieser 35 Tetraeder ist ebenfalls im Anhang zu finden. Als Auszug sind in der folgenden Tabelle die Tetraeder der vier reinen Elemente aufgelistet (Für den Laufparameter $\mathrm{S}$ in Gleichung (30) wurde der Wert von 0,15 mit Hinblick auf die späteren Ergebnisse eingesetzt.):

Tabelle 6: Berechnete Energien der vier ,,reinen“ Tetraeder im ZANC.

\begin{tabular}{l|l|l} 
Tetraeder & $\begin{array}{l}\text { ohne } \Delta \mathbf{E}_{\text {el }} \\
{[\mathbf{k J} / \mathbf{m o l}]}\end{array}$ & $\begin{array}{l}\text { mit } \Delta \mathbf{E}_{\mathbf{e l}} \\
{[\mathbf{k J} / \mathbf{m o l}]}\end{array}$ \\
\hline \hline $\mathrm{Zr} 4$ & $-59,52$ & $-50,49$ \\
$\mathrm{Ni4}$ & 16,68 & $-60,18$ \\
$\mathrm{Cu} 4$ & 49,04 & $-16,76$ \\
$\mathrm{~A} 14$ & 63,20 & 36,07
\end{tabular}

Auch hier hat wieder eine Verschiebung der Lösungsenergien stattgefunden. Man sieht, dass z.B. der Ni4 - Tetraeder bei Berücksichtigung der elastischen Energien sehr viel attraktiver ist, als im reinen Ni. Die Verschiebung ist jedoch für keinen der reinen Tetraeder so drastisch wie für den $\mathrm{Be}_{4}$-Tetraeder in den Vitreloy Legierungen. Die gesamte Zustandsdichte für ZANC mit $\mathrm{S}=0,15$ und $\sigma=4 \mathrm{~kJ} / \mathrm{mol}$ ist in der Abbildung $27 \mathrm{zu}$ sehen: 


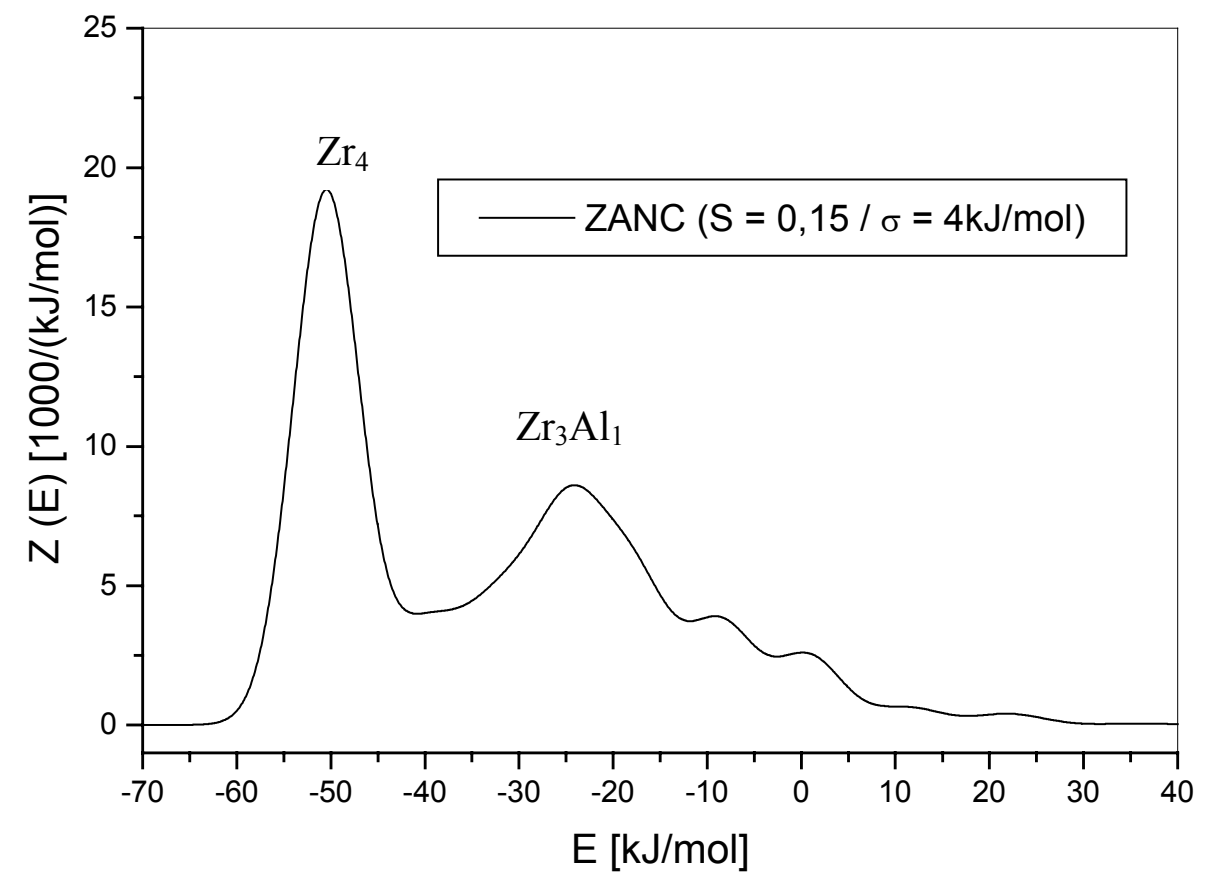

Abbildung 27: gesamte Zustandsdichte für ZANC. Die zur Berechnung benötigten Parameter sind zu entnehmen. Außerdem wurde $S=0,15$ und $\sigma=4 \mathrm{~kJ} / \mathrm{mol}$ gewählt.

Man erkennt in Abbildung 27 zwei Hauptmaxima: Das erste liegt bei $E=-50 \mathrm{~kJ} / \mathrm{mol}$ und wird überwiegend durch die $\mathrm{Zr}_{4}$-Tetraeder gebildet. Das zweite liegt bei $\mathrm{E}=-24 \mathrm{~kJ} / \mathrm{mol}$ und wird durch die $\mathrm{Zr}_{3} \mathrm{Al}_{1}$-Tetraeder gebildet. Die gesamten Plätze liegen innerhalb des Energieintervalls von $-70 \mathrm{~kJ} / \mathrm{mol}$ bis $+40 \mathrm{~kJ} / \mathrm{mol}$. Bei einer Vergrößerung des $\mathrm{S}-$ Parameters wird eine Verringerung der Höhe und eine Verschiebung der Energielage zu niedrigeren Werten des ersten Maximums erkennbar. Dies ist in Abbildung 28 für den Fall $\sigma=5 \mathrm{~kJ} / \mathrm{mol}$ dargestellt. Eine Variation der Halbwertsbreite $\sigma$ führt analog zum Vitreloy zu einer Veränderung der Maximahöhen. 


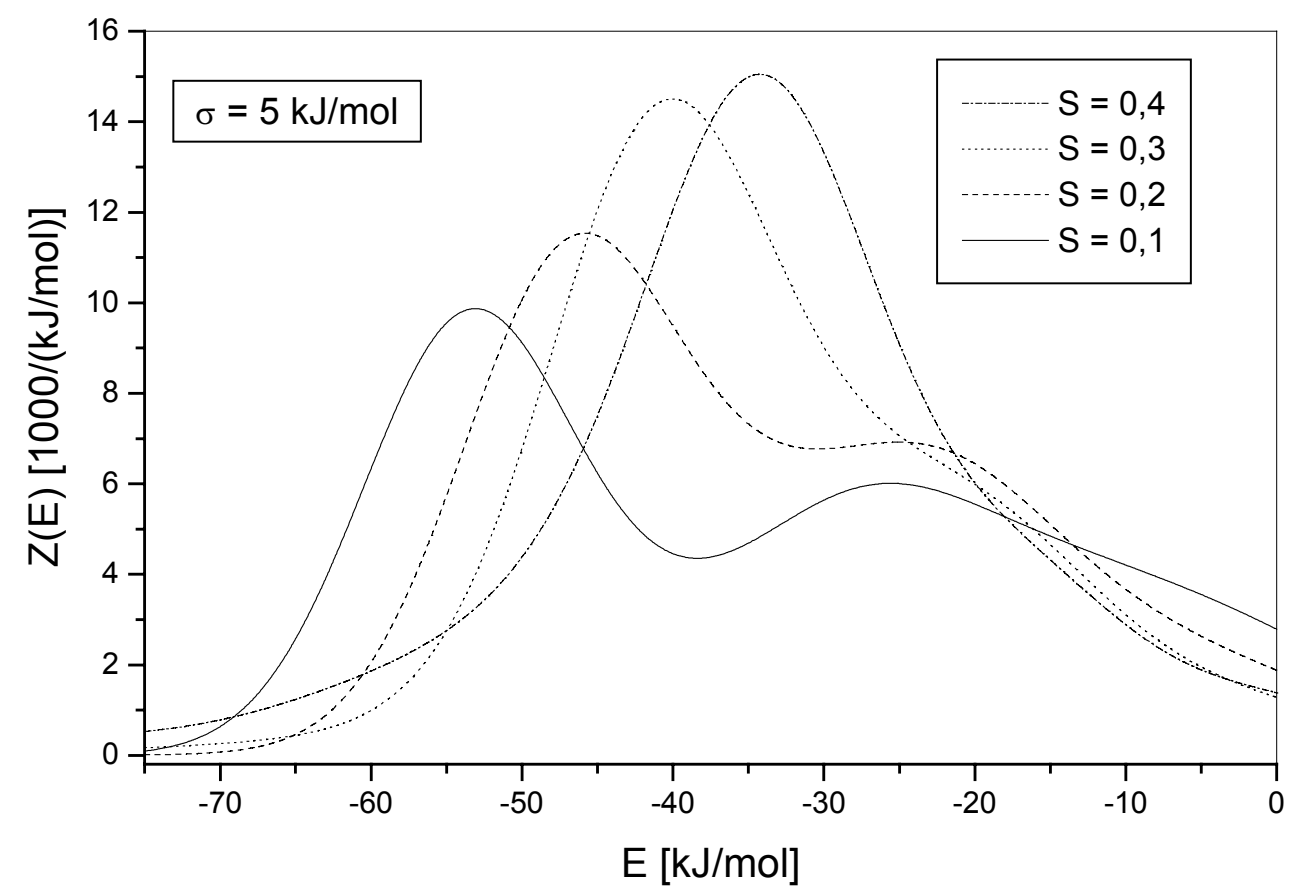

Abbildung 28: Variation des Parameters S bei der Berechnung der Zustandsdichten Z(E) für die Legierung ZANC. Die Berechnungen wurden alle mit einer Halbwertsbreite $\sigma=5 \mathrm{~kJ} / \mathrm{mol}$ durchgeführt. 


\subsection{Vergleich der Zustandsdichten aus Experiment und Modell}

Im folgenden Abschnitt werden die experimentell ermittelten Zustandsdichten aus Kapitel 4.1 für die Legierungen Vit 4, Vit 1 und ZANC mit den berechneten Zustandsdichten aus Kapitel 4.2 verglichen. Wie in Kapitel 2.1.2 beschrieben, wurden die experimentellen Z(E)-Verläufe mit Hilfe des Parameters f umgerechnet. Damit sind alle in diesem Kapitel dargestellten Zustandsdichten vom Typ “Tetraederplätze pro Energieintervall”. Die Anpassung der experimentellen Daten wurde durch eine geeignete Wahl der Parameter S (s. Kapitel 2.3.2), $\sigma$ (Halbwertsbreite der Gaußverteilung der Tetraeder) und dem Strukturfaktor f vorgenommen.

\subsubsection{Vitreloy-Legierungen}

Die Abbildung 29 und Abbildung 30 zeigen die Anpassung der drei Parameter $\mathrm{S}, \sigma$ und $\mathrm{f}$ an der Vit 4 - Legierung.

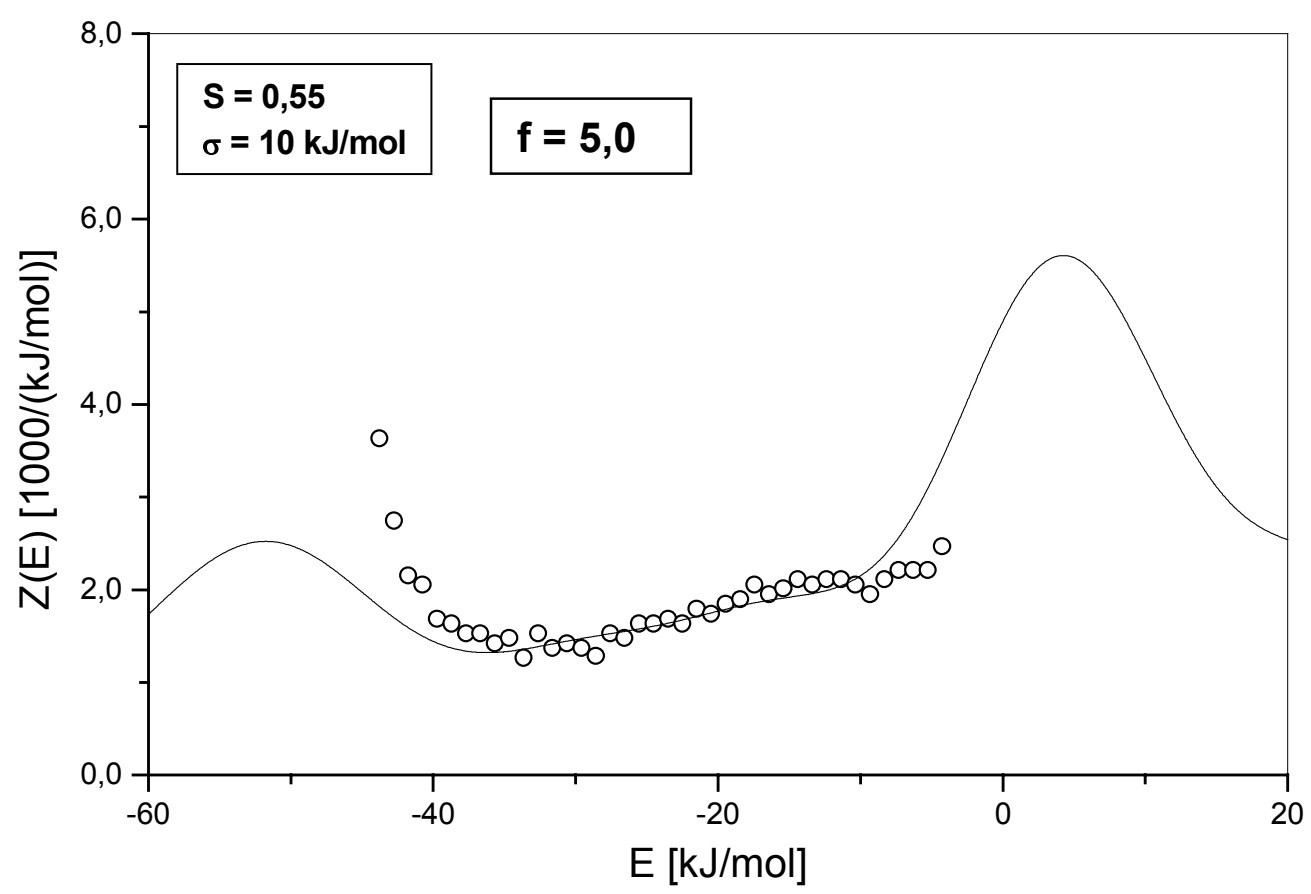

Abbildung 29: Anpassung der experimentell ermittelten und der berechneten Zustandsdichten für Vit 4. Hier: $S=0,55, \sigma=10 \mathrm{~kJ} / \mathrm{mol}$ und $f=5,0$ 


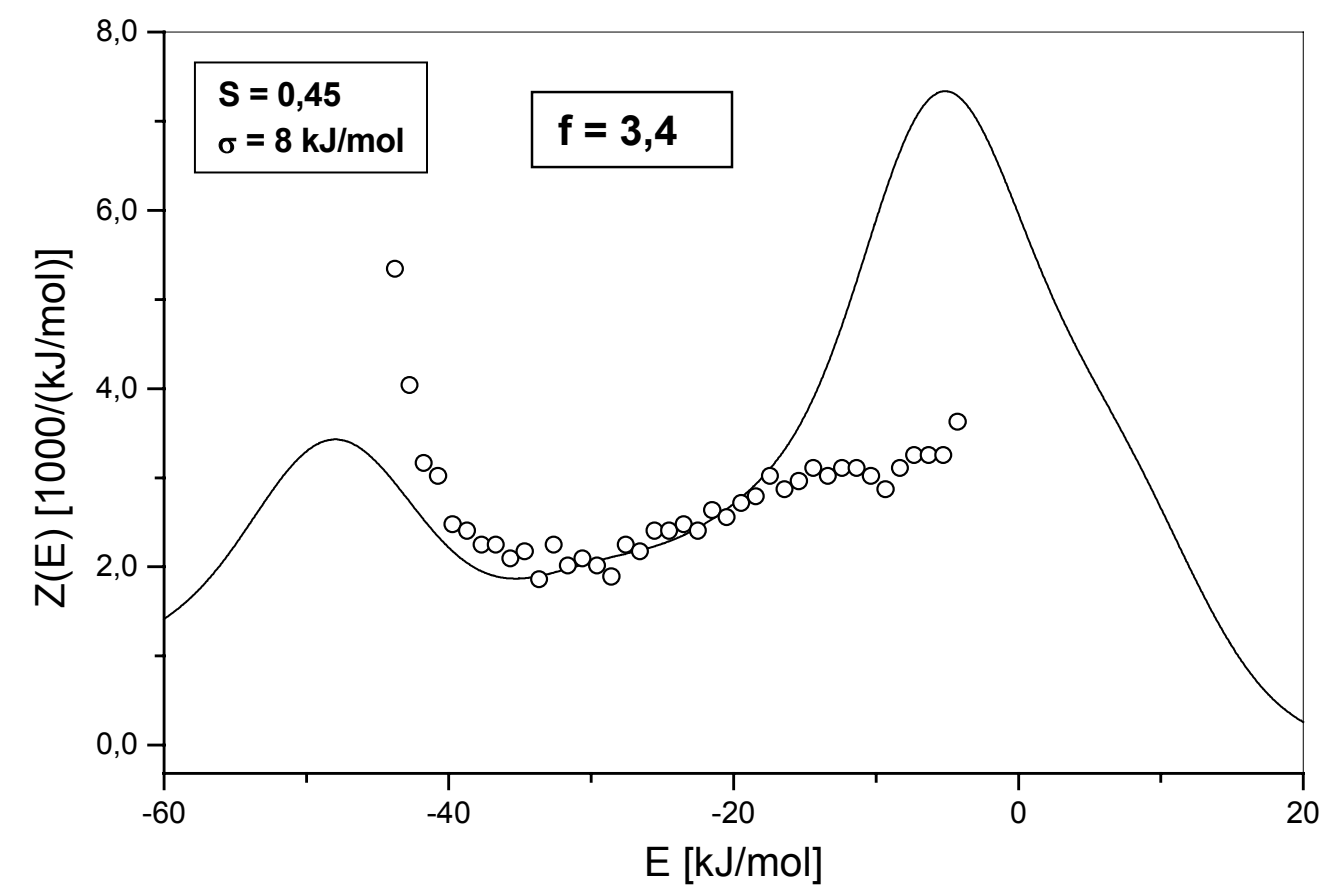

Abbildung 30: Anpassung der experimentell ermittelten und der berechneten Zustandsdichten für Vit 4. Hier: $S=0,45, \sigma=8 \mathrm{~kJ} / \mathrm{mol}$ und $f=3,4$

Da die experimentell ermittelten Zustandsdichten keine ausgeprägten Details zeigen, ist eine eindeutige Anpassung der drei Parameter nicht möglich. Eine Anpassung wird erreicht, wenn $\mathrm{S}$ zwischen 0,45 und $0,55, \sigma$ zwischen $8 \mathrm{~kJ} / \mathrm{mol}$ und $10 \mathrm{~kJ} / \mathrm{mol}$ und $\mathrm{f}$ zwischen 3,4 und 5,0 liegt. Kleine $\mathrm{S}-$ Werte führen zu einem schmaleren Minimum der Zustandsdichte, bei zu hohen S - Werten treten Nebenmaxima auf. Für die Halbwertsbreiten wurde festgestellt, dass kleinere $\sigma$-Werte als $8 \mathrm{~kJ} / \mathrm{mol}$ (bei $\mathrm{S}=0,45$ ) bzw. $10 \mathrm{~kJ} / \mathrm{mol}$ (bei $\mathrm{S}=0,55$ ) zwar die anfängliche große negative Steigung etwas besser annähern, aber gleichzeitig dazu führen, dass sich wieder die erwähnten zusätzlichen Maxima innerhalb des großen Minimums ausprägen. Es ist auch verständlich, dass bei der Kurve mit $S=0,55$ bereits $\sigma$-Werte kleiner als $10 \mathrm{~kJ} / \mathrm{mol}$ ausreichen: Diese Zustandsdichte ist im Vergleich zu der Zustandsdichte mit $\mathrm{S}=0,45$ weiter auseinandergezogen und die einzelnen Tetraederenergieverteilungen sind weiter voneinander separiert. Dadurch sind bereits etwas breitere Maxima von einander unterscheidbar. 
Analoges gilt für die Anpassung bei der Vit 1-Legierung, die in Abbildung 31 und Abbildung 32 gezeigt ist. Die besten Anpassung gelang mit den Parametern $\mathrm{S}=0,55$, $\sigma=12 \mathrm{~kJ} / \mathrm{mol}$ und $\mathrm{f}=4,7$ für den in Abbildung 31 gezeigten Fall. In die Abbildung 32 wurden die Parameter $\mathrm{S}=0,60, \sigma=14 \mathrm{~kJ} / \mathrm{mol}$ und $\mathrm{f}=5,6$ verwendet. Kleinere $\sigma$-Werte sorgten in der berechneten Zustandsdichte für eine zu große Steigung der Flanke des Peaks und machten eine Anpassung der Kurven nicht mehr möglich. Zu große $\sigma$-Werte sorgten für zu flache Kurvenverläufe. Die Position des Peaks wird durch größere oder kleiner S Parameter ebenfalls derart verschoben, dass eine Übereinstimmung zwischen Experiment und Modell nicht mehr zu finden war.

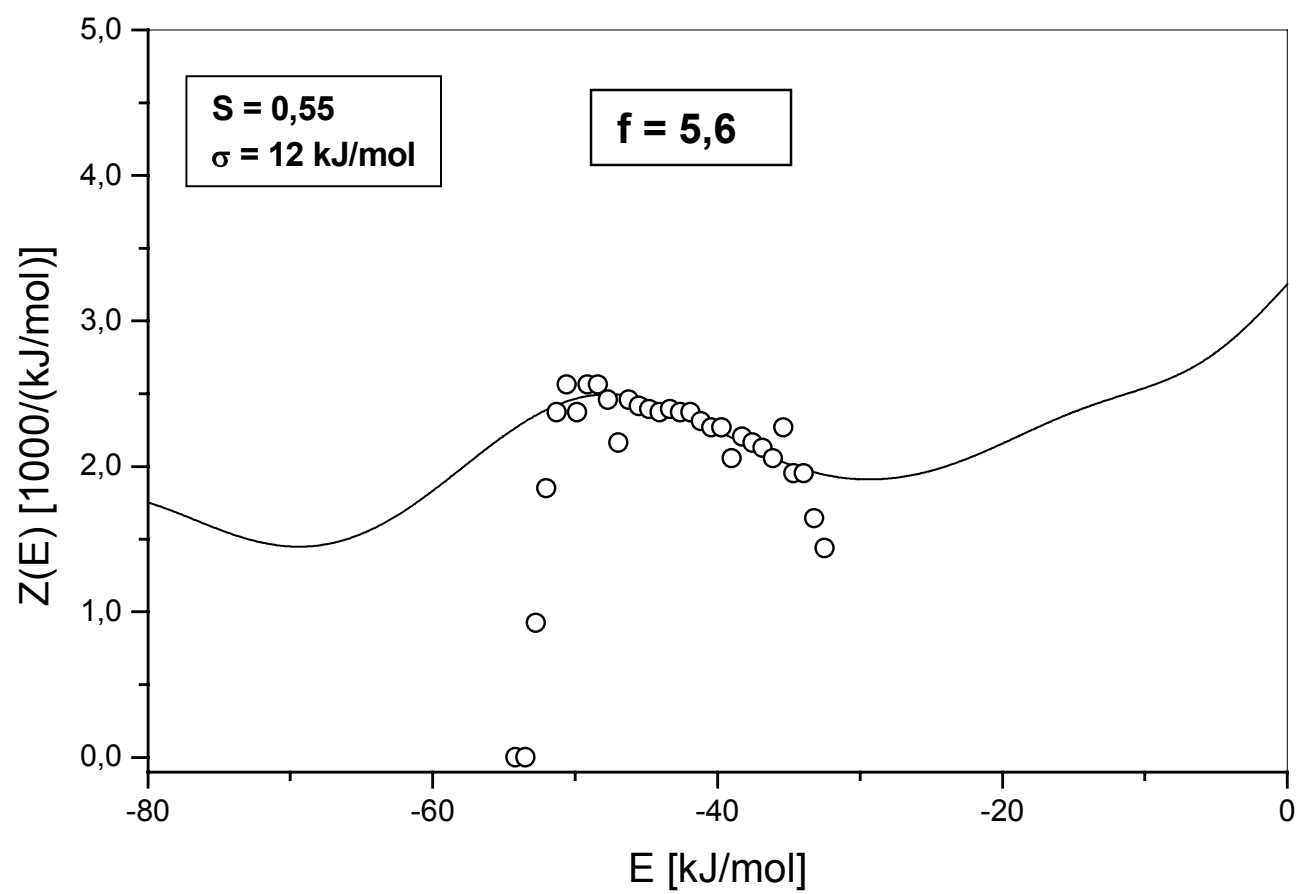

Abbildung 31: Anpassung der experimentell ermittelten und der berechneten Zustandsdichten für Vit 1. Hier: $S=0,55, \sigma=12 \mathrm{~kJ} / \mathrm{mol}$ und $f=5,6$ 


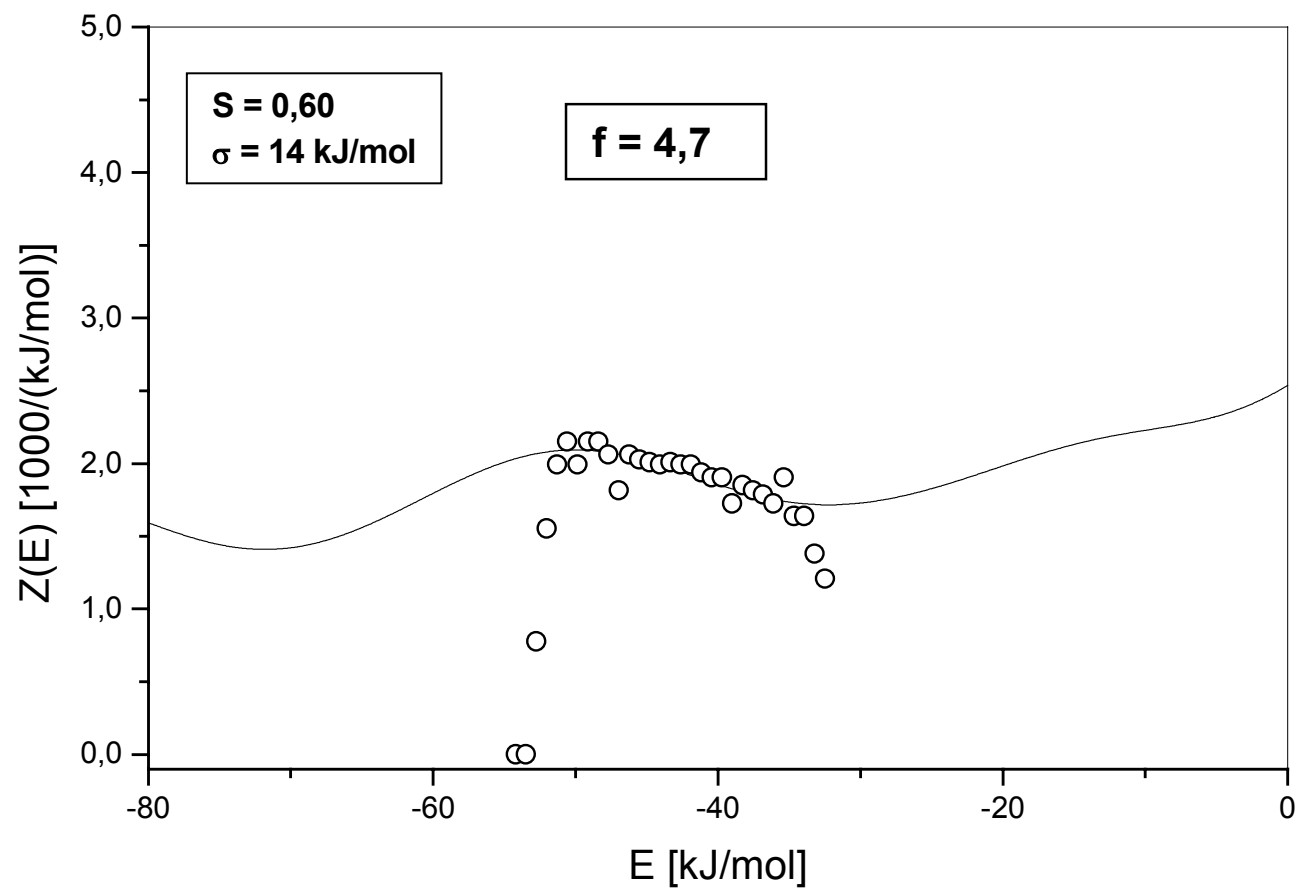

Abbildung 32: Anpassung der experimentell ermittelten und der berechneten Zustandsdichten für Vit 1. Hier: $S=0,55, \sigma=12 \mathrm{~kJ} / \mathrm{mol}$ und $f=5,6$

\subsubsection{ZANC-Legierung}

Die aus den Nicht-Gleichgewichts-Isothermen experimentell bestimmten und theoretischen Zustandsdichten für die ZANC - Legierung sind in der Abbildung 33 zu sehen. Die besten Übereinstimmungen konnten mit den Werten $\mathrm{S}=0,15$ und $\mathrm{f}=2,5$ für die Halbwertsbreiten $\sigma=3 \mathrm{~kJ} / \mathrm{mol}$ und $\sigma=4 \mathrm{~kJ} / \mathrm{mol}$ erzielt werden. 


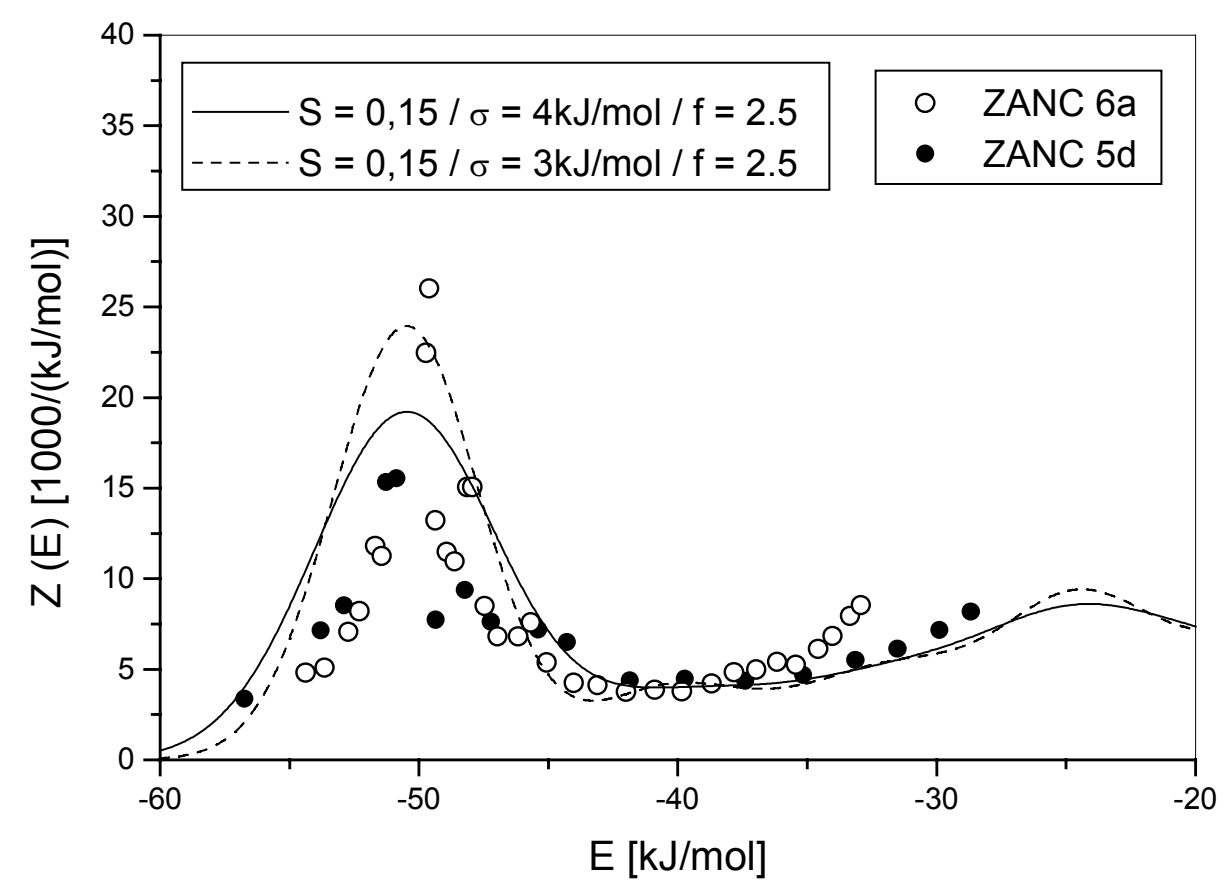

Abbildung 33: Experimentell ermittelte Zustandsdichten an den ZANC-Proben ZANC-6a und ZANC-5d (Kreise). Die durch Linien dargestellten berechneten Z(E)-Verläufe stellen die bestmögliche Anpassung der experimentellen Daten mit den berechneten dar. Die verwendeten Parameter sind in der Abbildung zu sehen.

Mit $\mathrm{S}=0,15$ stimmt die Position des ersten Maximums in der berechneten $\mathrm{Z}(\mathrm{E})$ mit dem in der experimentell ermittelten Zustandsdichte überein. Der Vergleich der Peakbreiten dieses Maximums bei $\mathrm{E}=-50 \mathrm{~kJ} / \mathrm{mol}$ zeigt jedoch, dass $\sigma$ noch kleiner als $3 \mathrm{~kJ} / \mathrm{mol}$ sein müsste, um das gemessene Plateau in Abbildung 22 beschreiben zu können.

Die Zustandsdichte aus der Gleichgewichtesisotherme ist in Abbildung $34 \mathrm{zu}$ sehen. Eine gute Anpassung der experimentell ermittelten und theoretischen Kurvenverläufe war mit den Parametern $\mathrm{S}=0,1, \sigma=5 \mathrm{~kJ}$ und $\mathrm{f}=3$ möglich. 


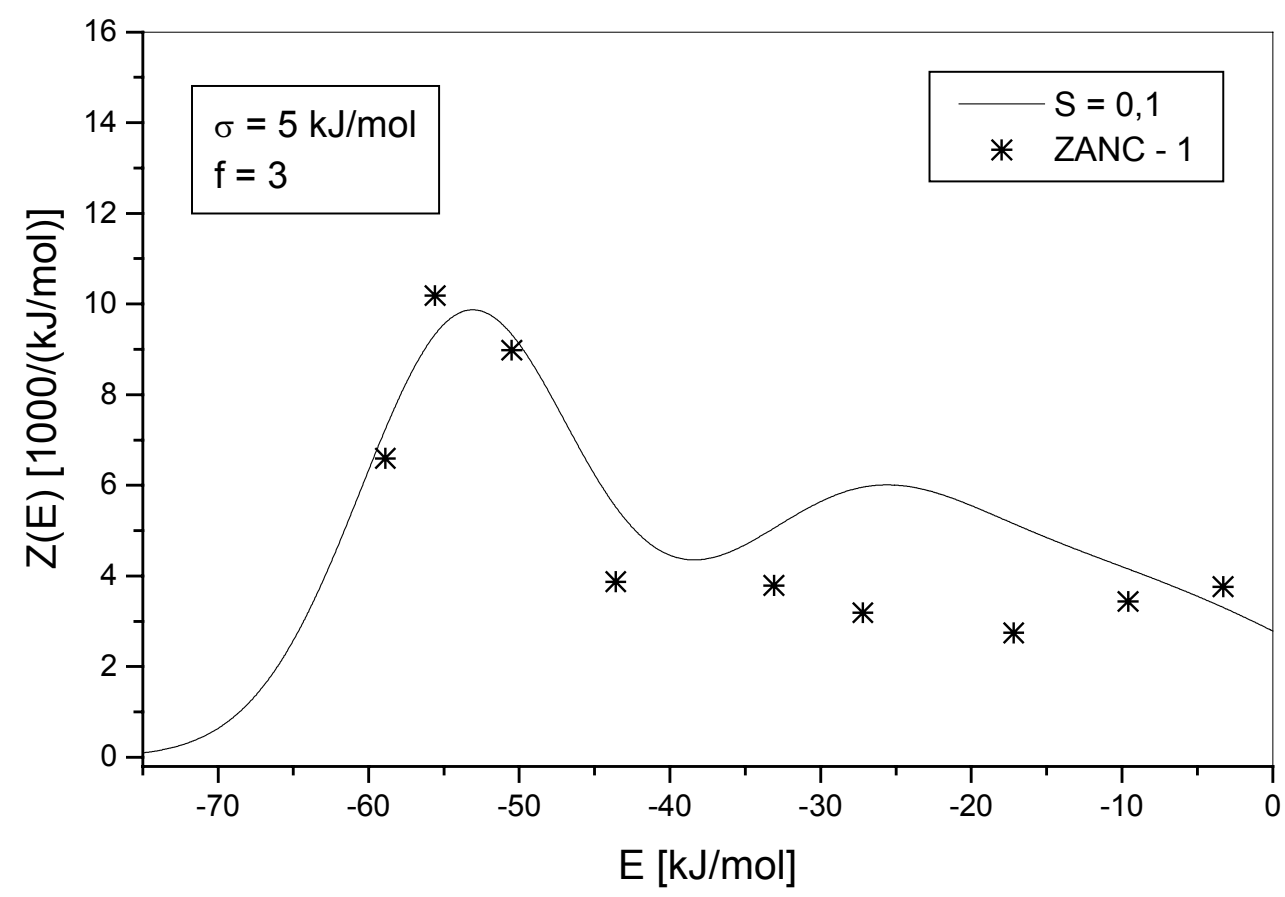

Abbildung 34: experimentell ermittelte Zustandsdichten an der ZANC-Probe ZANC-1. Die verwendeten Parameter sind in der Abbildung zu sehen.

Abschließend ist in der Abbildung 35 noch ein Vergleich zwischen Vit 4 und ZANC zu sehen. Die Zustandsdichte für ZANC wurde mit $\mathrm{S}=0,15$ und $\sigma=5 \mathrm{~kJ} / \mathrm{mol}$ angepasst, die von Vit 1 mit $\mathrm{S}=0,35$ und $\sigma=6 \mathrm{~kJ} / \mathrm{mol}$. Z(E) von ZANC ist lokalisiert zwischen $-60 \mathrm{~kJ} / \mathrm{mol}$ und 20kJ/mol. Die Vit 1 Verteilung erstreckt sich jedoch bis hin zu sehr niedrigen Energien. Dieser Effekt hängt mit den Atomradien der Legierungspartner zusammen. 


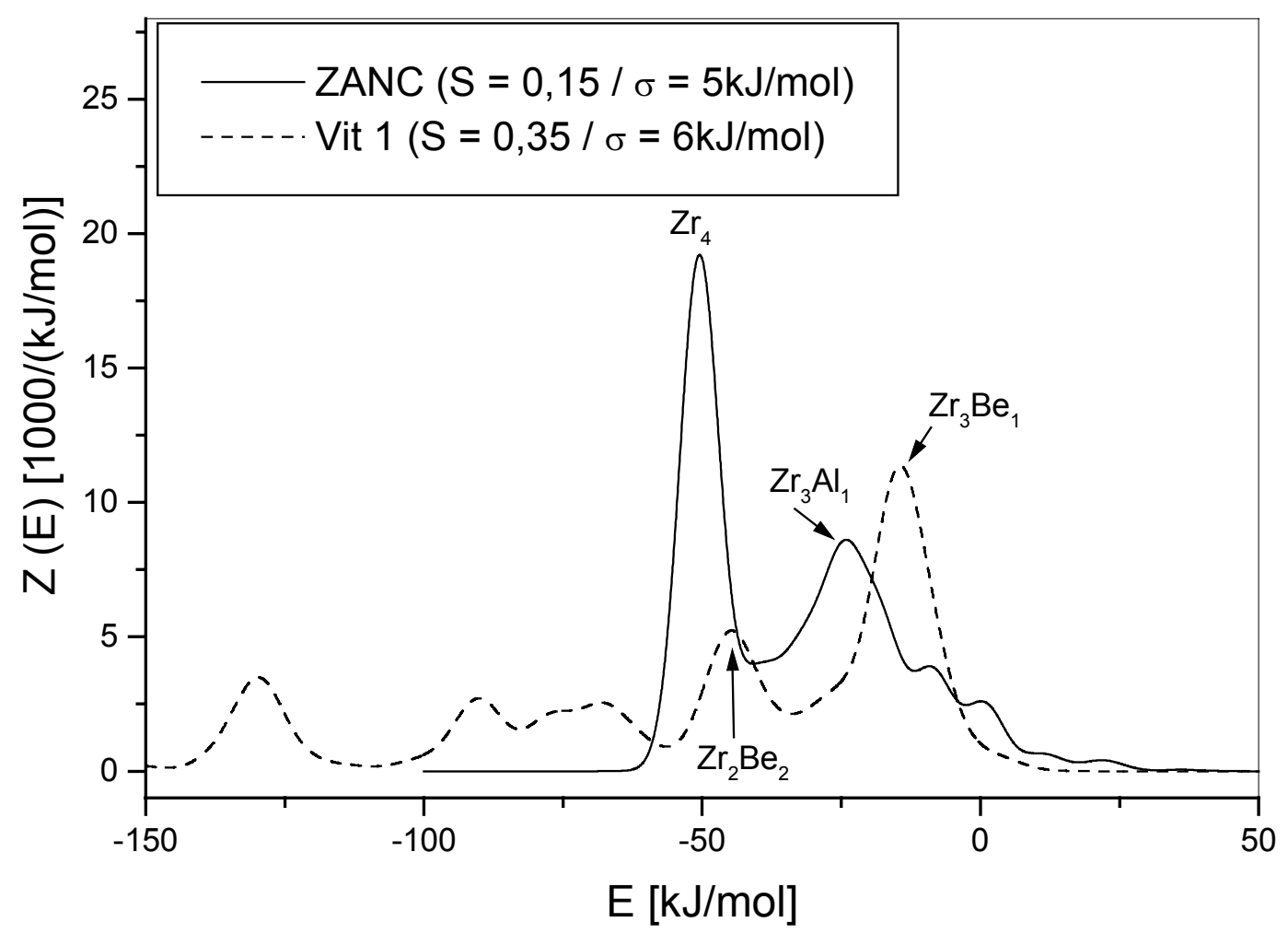

Abbildung 35: experimentell ermittelte Zustandsdichten an der ZANC-Probe ZANC-1. Die verwendeten Parameter sind in der Abbildung zu sehen. 


\subsection{Ergebnisse der Diffusionsexperimente}

Im Kapitel 3.2.2 wurde ein neue Methode vorgestellt, die es ermöglicht Durchbruchszeiten im Sinne von Permeationsmessungen zur Bestimmung von Diffusionskoeffizienten für Wasserstoff in amorphen dünnen Schichten zu messen. Dies geschieht durch die Beobachtung einer durch Wasserstoff induzierten Widerstandsänderung in einer Pd-Leitschicht, auf die die zu untersuchende Schicht aufgebracht ist. Nachdem der Wasserstoff durch diese Schicht diffundiert ist, löst er sich in der darunter liegenden Pd-Leitschicht und bewirkt dort eine signifikante Widerstandsänderung. Der zeitliche Verlauf der Widerstandsänderung ist ein direktes Maß für die Wasserstoffdiffusion in der amorphen Schicht. Mit Hilfe dieser Methode kann bei festgehaltener Schichtdicke die Temperatur-, Druck- und Wasserstoffkonzentrationsabhängigkeiten des Diffusionskoeffizienten bestimmt werden. Als ein Beispiel zeigt die Abbildung 36 die Abhängigkeit der Durchbruchszeit vom Wasserstoffdruck in einer doppellogarithmischen Auftragung:

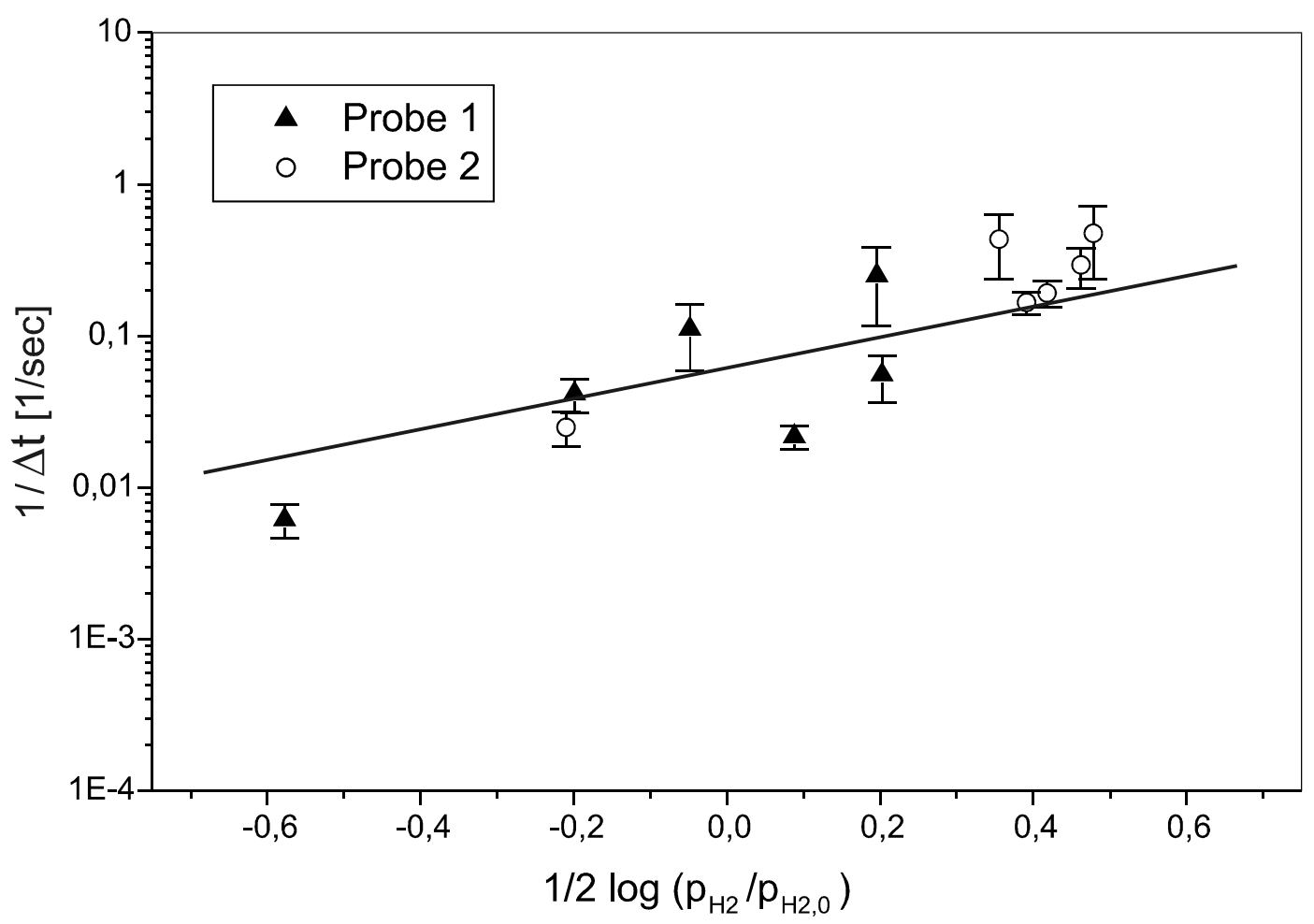

Abbildung 36: Reziproke Durchbruchszeiten an Proben mit einer 50nm amorphen Schicht als Funktion des Logarithmus des Wasserstoffdruckes. Die eingezeichente Gerade hat die Steigung 1 
Die amorphen Schichten waren hier 50nm dick. Vor jeder Beladung wurden die Proben durch Abpumpen des Rezipienten vom Wasserstoff entleert. Der eingestellte Wasserstoffdruck während der Beladung wurde konstant gehalten. Von Messpunkt zu Messpunkt wurde der eingestellte Druck jedoch zwischen $\mathrm{p}_{\mathrm{H} 2}=0,05$ mbar und $\mathrm{p}_{\mathrm{H} 2}=9$ mbar variiert. Die Durchbruchszeiten wurden in der in Abbildung 10 gezeigten Weise bestimmt. Die in Abbildung 36 gewählte Darstellung der Messdaten beruht auf Gleichung (40), nach $\operatorname{der} D_{\text {in }} \propto 1 / \Delta t$ und auf Gleichung (15), nach der $D_{\text {in }}$ eine Funktion von $\left(\mathrm{p}_{\mathrm{H} 2}\right)^{1 / 2}$ ist. Die in Abbildung 36 eingezeichnete Gerade der Steigung 1 entspricht der Proportionalität $\mathrm{D}_{\mathrm{in}} \propto\left(\mathrm{p}_{\mathrm{H} 2}\right)^{1 / 2}$ (s. auch Kapitel 5.3.3).

Die Grenzen dieses Messverfahrens zeigt die Abbildung 37: Der Widerstand einer Pd-Referenzschicht wird synchron mit einer Pd-Leitschicht gemessen, auf der sich eine $150 \mathrm{~nm}$ dicke amorphe Legierungsschicht befindet. Insgesamt wurde nach dem erstmaligem Wasserstoffeinlass der Druck in dem Rezipienten, in dem sich beide Proben befanden, noch zwei mal erhöht. In der ersten Beladung (*) ist eine Widerstandsänderung, wie sie nach Kapitel 3.2.2 für ein Permeationsexperiment zu erwarten ist, in der Probe mit der amorphen Deckschicht zu beobachten. Doch schon bei einer zweiten Beladung verläuft die Änderung des Widerstandes identisch mit der reinen Pd-Referenzschicht. 


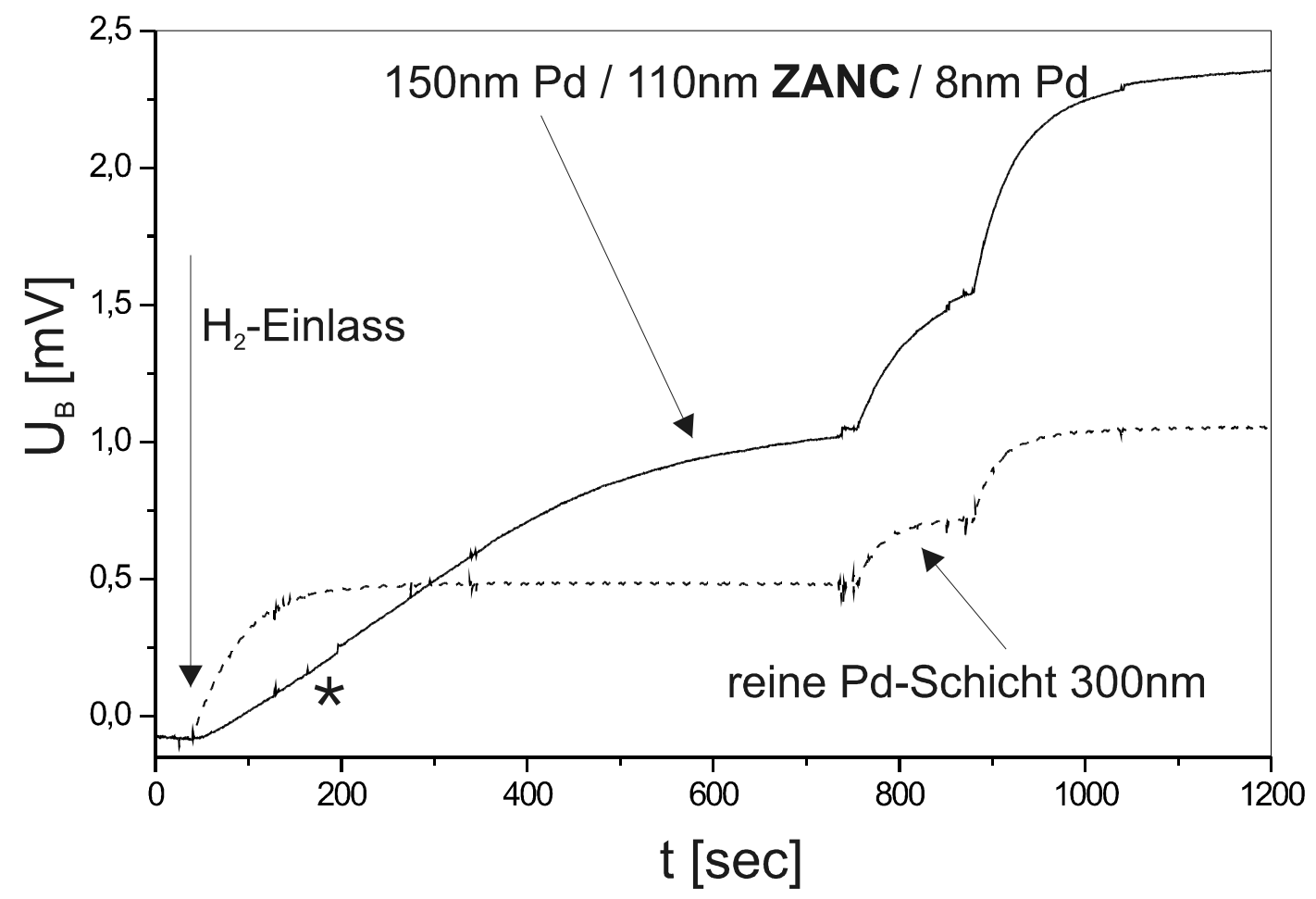

Abbildung 37: Vergleich der Widerstandsänderungen bei Wasserstoffbeladung zwischen einer reinen Pd-Referenzschicht und einem Pd/Glas/Pd-Schichtpaket. Das Verhältnis der Sättigungswiderstände entspricht dem inversen Verhältnis der Pd-Schichtdicken. Der Einfluss der amorphen Schicht auf den Gesamtwiderstand ist daher sehr gering.

Schon die erste Widerstandsänderung, die offensichtlich einen Einfluss der amorphen Schicht auf die Wasserstoffaufnahme im Pd zeigt, kann jedoch nicht zur Bestimmung von Diffusionseigenschaften verwendet werden. Die Auftragung der Durchbruchszeiten gegen die Schichtdicke der amorphen Deckschicht (bei gleichbleibenden Pd-Schichtdicken) in Abbildung 38 zeigt eine unphysikalische Abnahme der Durchbruchszeiten mit zunehmender Schichtdicke. 


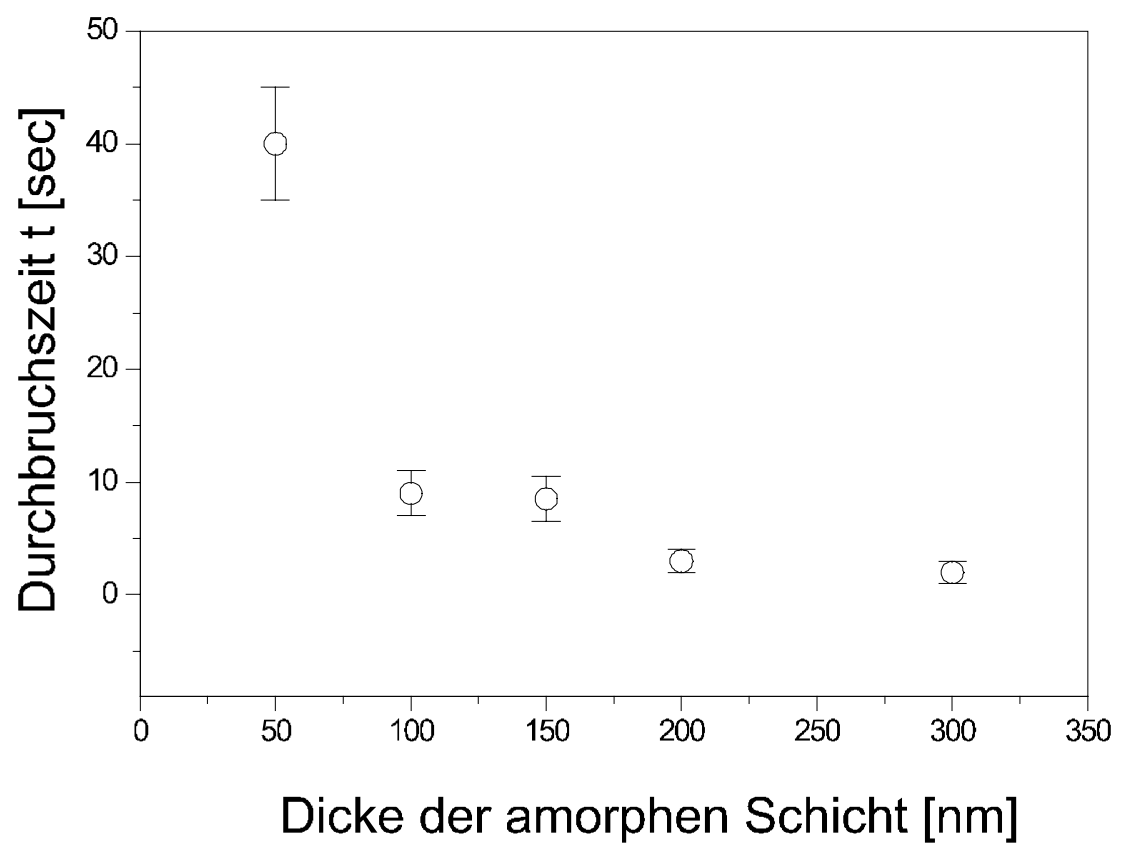

Abbildung 38: Beobachtete Abhängigkeit der Durchbruchszeiten von der Dicke der amorphen Zwischenschicht.

Die Gründe für dieses anormale Verhalten liegt in einer Ablösung der Schichten begründet. Dabei zeigt Abbildung 38 dass die Filme umso stabiler sind, je dünner die amorphe Schicht ist. Es war eine kritische Schichtdicke zu erwarten, unterhalb derer die Schichtsysteme stabil bleiben sollten und ein reproduzierbares Beladungsverhalten möglich ist. Es wurde herausgefunden, dass mit Proben deren amorphe Schicht $50 \mathrm{~nm}$ dick war, die Widerstandsänderungen reproduzierbar gemessen werden konnten. Die Reproduzierbarkeit der Widerstandsänderungen entsprechend Abbildung 10 ist notwendig, um sicher zu sein, dass nur diffusionskontrollierte Durchbruchszeiten bestimmt werden. Die in Abbildung 36 gezeigten Messungen sind an zwei Proben gemessen worden, deren amorphe Schichten eine Dicke von $50 \mathrm{~nm}$ besaßen. Die Reproduzierbarkeit ist in der Abbildung 39 für drei Messungen an einer einzigen Probe für drei verschiedene Drücke demonstriert. 


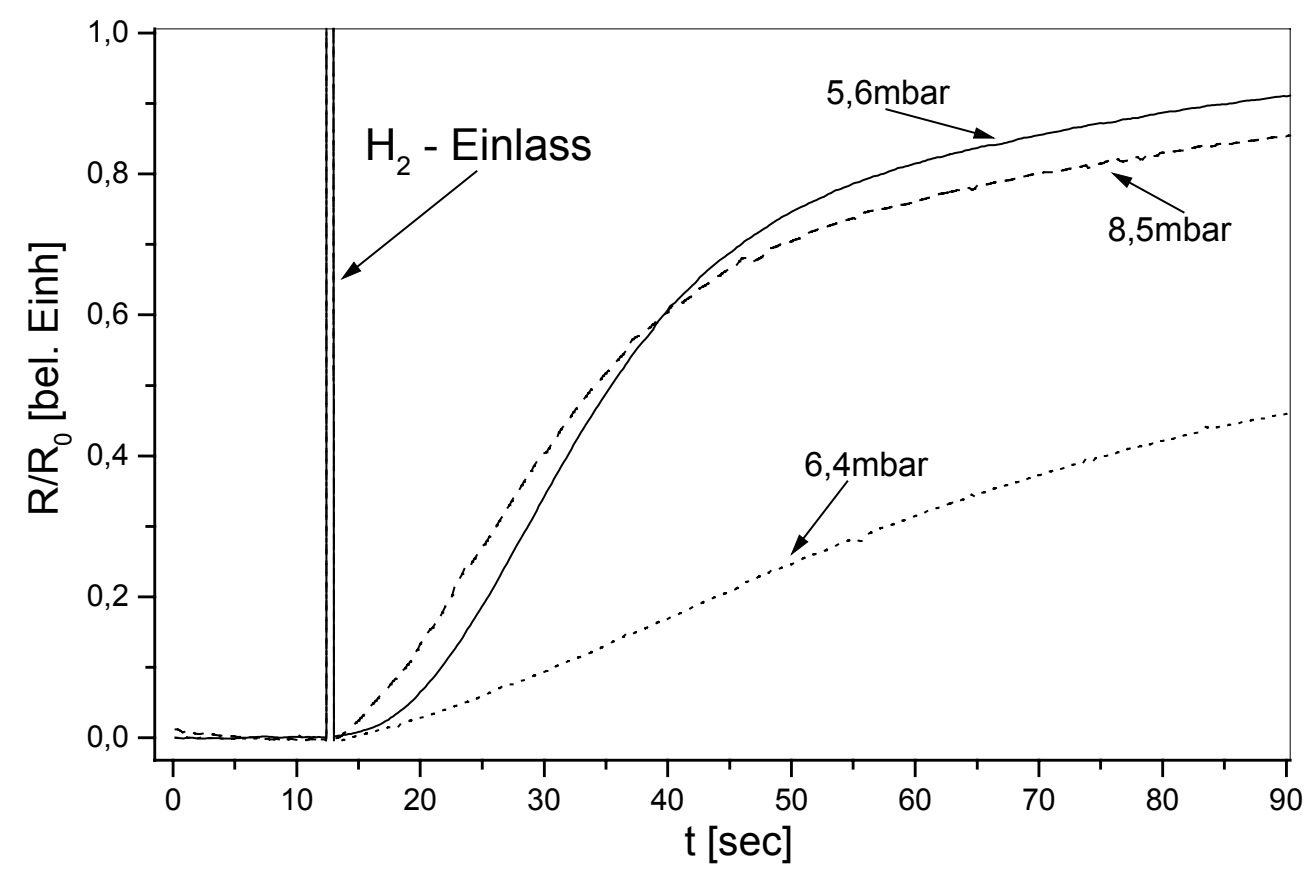

Abbildung 39: Drei Widerstandsänderungen an einem Schichtsystem mit einer $50 \mathrm{~nm}$ dicken amorphen Schicht bei drei verschiedenen Drücken beladen. (Die unterschiedlichen und vom Druck nicht her zu erwartenden Widerstandswerte sind eine Folge unterschiedlicher Spannungen an der Brückenschaltung)

Das Ablöseverhalten der Schichten konnte auch in den lichtmikroskopischen Aufnahmen der Abbildung 40 beobachtet werden. Es sind dort die Oberflächen zweier amorpher Filme abgebildet, die beide bei einem Wasserstoffdruck von $\mathrm{p}_{\mathrm{H} 2}=100$ mbar beladen worden sind. Die Dicke der Schichten betrug s $=300 \mathrm{~nm}$ (Abbildung 40-a) und $\mathrm{s}=200 \mathrm{~nm}$ (Abbildung 40-b). Die $300 \mathrm{~nm}$ dicke Schicht bildet, obwohl er ebenso wie die $200 \mathrm{~nm}$ dicke Schicht dem gleichen Wasserstoffdruck ausgesetzt war, wesentlich größere Blasen und Falten an der Oberfläche. Bei der $200 \mathrm{~nm}$ dicken Schicht sind lediglich nur noch kleine Bläschen zu finden. 


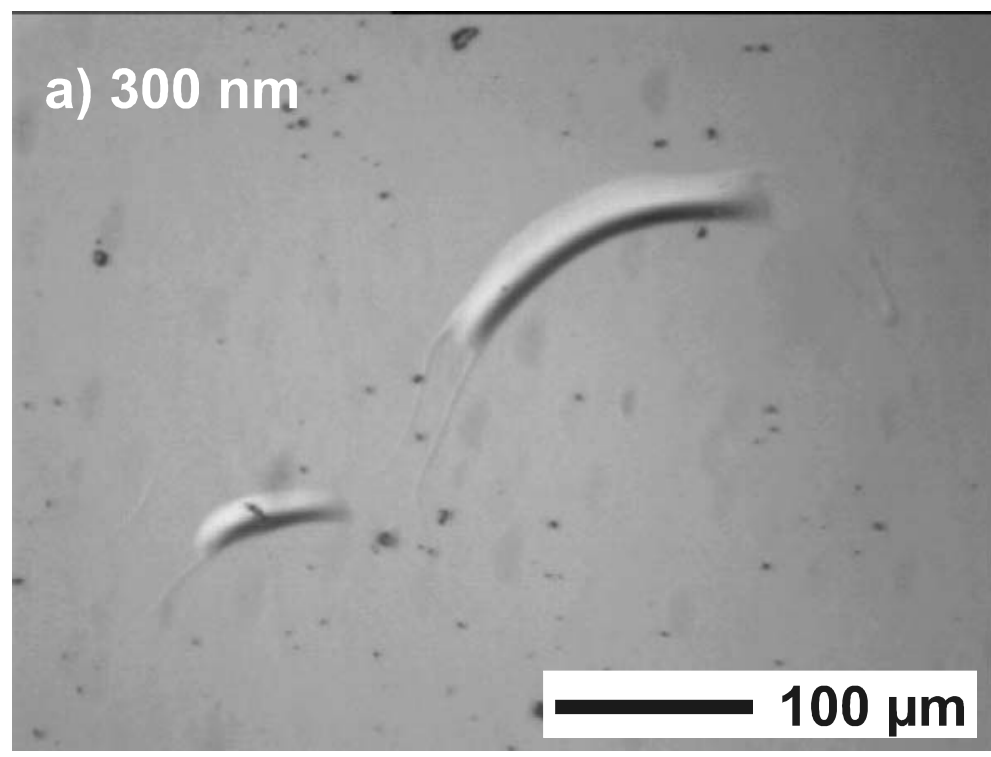

\section{b) $200 \mathrm{~nm}$}

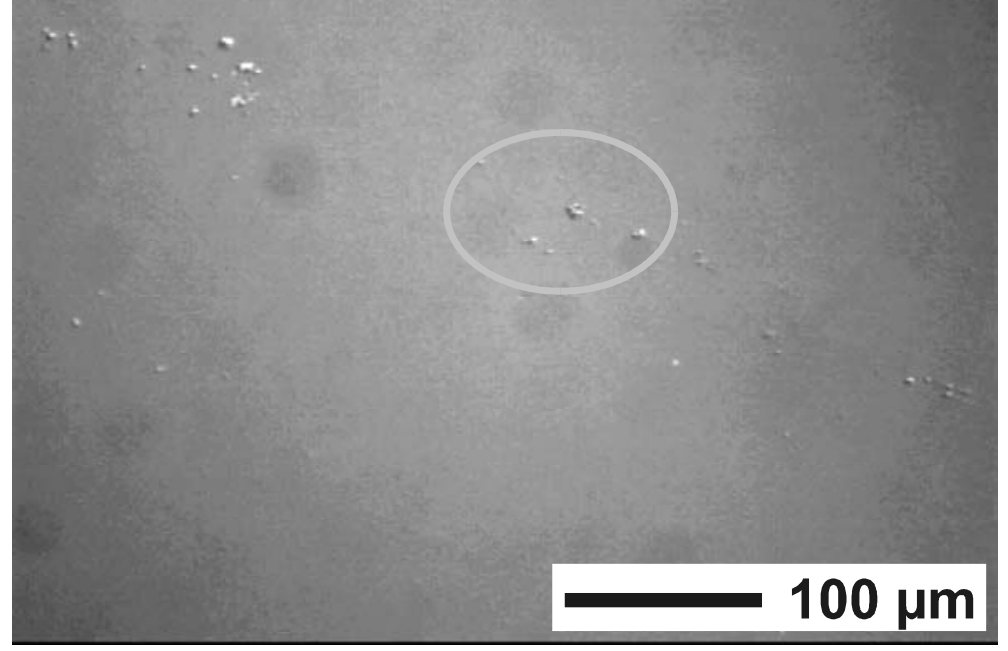

Abbildung 40: Lichtmikroskopische Aufnahmen der Oberfläche zweier ZANC-Schichten mit der in Abbildung 12 vorgestellten Probengeometrie. a) $60 \mathrm{~nm} P d+300 \mathrm{~nm} Z A N C+8 \mathrm{~nm} P d$ und b) $60 \mathrm{~nm} P d+200 \mathrm{~nm} Z A N C+$

$8 \mathrm{~nm}$ Pd. Beide Proben wurden dem selben Druck von 100mbar Wasserstoff ausgesetzt. Deutlich ist eine stärkere Ablösung der dickeren Schicht zu erkennen. 


\section{Diskussion der Ergebnisse}

\subsection{Zustandsdichten an Vit 1, Vit 4}

Die Werte für $\mathrm{S}, \sigma$ und $\mathrm{f}$, wie sie in dieser Arbeit für die Legierungen Vit 1 und Vit 4 in Kapitel 4.3 bestimmt wurden, sind Tabelle 7 noch einmal zusammengefasst:

Tabelle 7: Ergebnisse der Anpassung der experimentellen und theoretischen Zustandsdichten.

\begin{tabular}{|c|c|c|}
\hline & Vit 4 & Vit 1 \\
\hline $\mathrm{S}$ & 0,45 bis 0,55 & 0,55 bis 0,60 \\
\hline$\sigma[\mathrm{kJ} / \mathrm{mol}]$ & 8 bis 10 & 12 bis 14 \\
\hline $\mathrm{f}$ & 3,4 bis 5,0 & 4,7 bis 5,6 \\
\hline
\end{tabular}

Die gefundenen Werte von S liegen sehr nahe an dem Wert von 0,52, den [Bro89a] für eine gestörte $\mathrm{NbV}$-Legierung bestimmt hat. Jedoch ist eine wesentliche Konsequenz dieses Parametersatzes, dass eine große Anzahl von Zuständen niedriger Energien existieren, die im Vitreloy zu den Be-reichen Tetraedern korrespondieren und in denen der Wasserstoff quasi kovalent gebunden wäre. Die Anpassung an die experimentellen Daten führt dann zu Werten für $\mathrm{f}$, die $\mathrm{zu}$ groß sind. Wie bereits in Kapitel 2.1.2 erwähnt, ist in einer dichtestgepackten fcc-Struktur $\mathrm{f}=3$. Je größer $\mathrm{f}$ ist, desto niedriger verläuft die berechnete Z(E) Kurve im Vergleich zur experimentell bestimmten. Integriert man die Zustandsdichte in Abbildung 41 bis $\mathrm{E} \approx-70 \mathrm{~kJ} / \mathrm{mol}$ und multipliziert diesen Wert mit dem hier bestimmten Faktor $\mathrm{f}=3,4$, so erhält man die Konzentration $\mathrm{c}_{\mathrm{H}, \mathrm{At}}=0,54 \mathrm{H} / \mathrm{Me}$. Dieser Wert ist viel $\mathrm{zu}$ hoch. Da eine Energie von $\mathrm{E}=-70 \mathrm{~kJ} / \mathrm{mol}$ einem Druck von ungefähr $\mathrm{p}_{\mathrm{H} 2}=10^{-25} \mathrm{bar}$ entspricht, und der Wasserstoffpartialdruck in der Atmosphäre bereits höher als $10^{-10} \mathrm{bar}$ ist, sollte eine Probe mit einer wie in Abbildung 41 gezeigten Zustandsdichte stets mit einer Startkonzentration von mindestens $\mathrm{c}_{\mathrm{H}} \approx 0,50 \mathrm{H} / \mathrm{Me}$ vorliegen. Es gibt jedoch keinerlei 
Hinweise dafür, dass diese hohen Startkonzentrationen tatsächlich vorliegen und beobachtet worden sind.

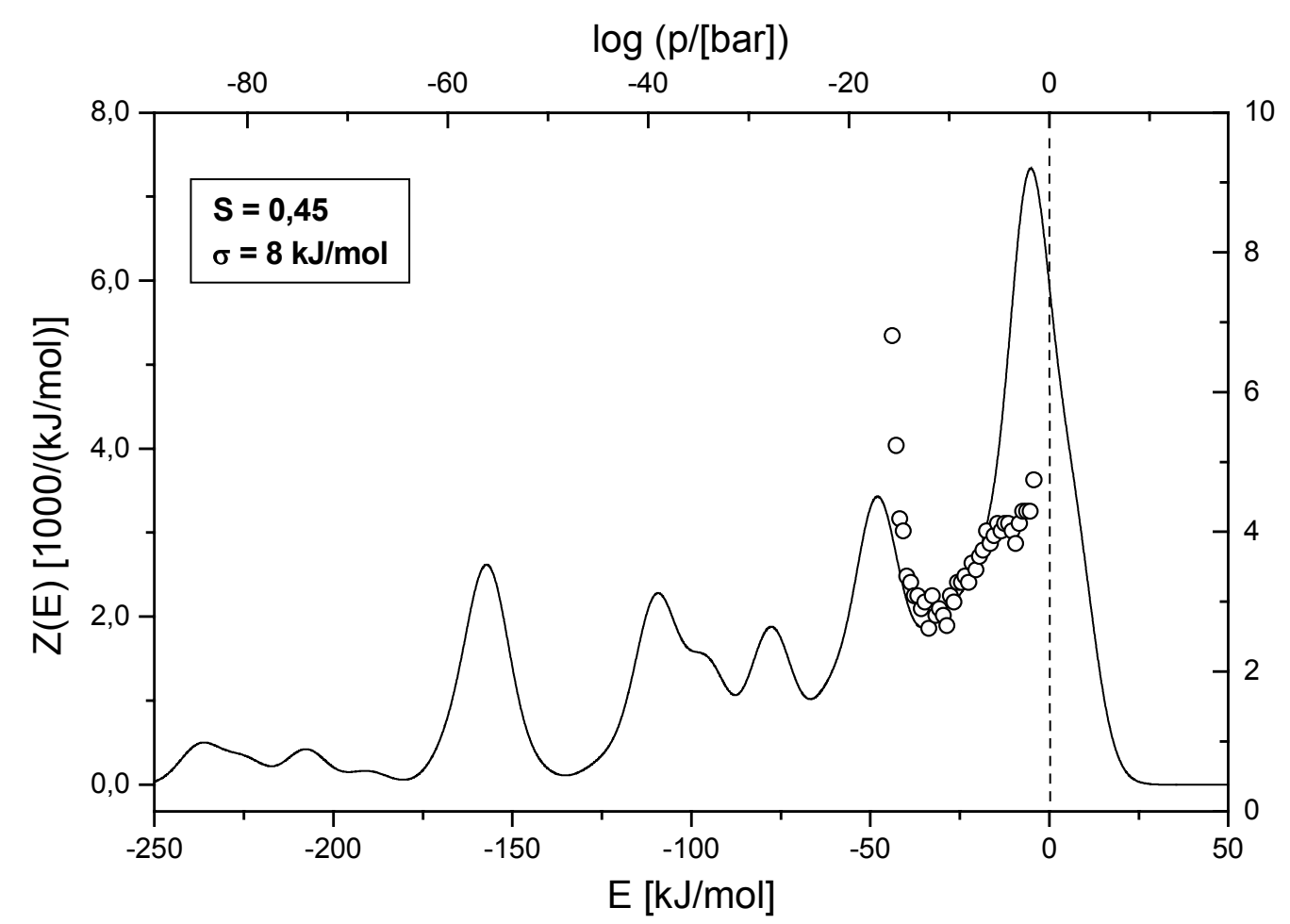

Abbildung 41: berechnete und experimentelle Zustandsdichte für Vit 4 (hier: $f=3,4$ ). Das dargestellte Energieintervall ist wesentlich größer als in den vorigen Abbildungen.

Trotzdem muss davon ausgegangen werden, dass diese nach den Berechnungen tiefliegenden Tetraeder zumindest von der Zusammensetzung her existieren, jedoch mit in jedem Fall höheren Platzenergien. Eine in erster Näherung gleichmäßige Verteilung dieser Plätze auf den höherliegenden Bereich der Zustandsdichte erhöht den Verlauf der Zustandsdichte und erfordert somit einen kleineren Parameter $f$, als in dieser Arbeit bestimmt worden ist. Die Diskrepanz zwischen dem erwarteten und hier bestimmten fWerten kann somit erklärt werden.

Die Ursache für das Auftreten so niederenergetischer Plätze folgt aus der Annahme, dass alle Tetraedertypen durch einen einzigen $\mathrm{S}$ - Parameter beschrieben werden können. Dies sorgt dafür, dass die Größen der simulierten Tetraederplätze in der amorphen Legierung alle in gleichem Maße zwischen dem mittlerem Volumen $<\mathrm{V}>$ und den entsprechenden freien 
Tetraederplatzgrößen $\mathrm{V}^{0}{ }_{\mathrm{i}}$ verschoben sind (s. Abbildung 5). In Kapitel 2.3.2 wurde bereits darauf hingewiesen, dass ein $\mathrm{S}=0,5$ z.B. bedeutet, dass alle Volumina der amorphen Tetraeder genau in der Mitte von $\left\langle\mathrm{V}>\right.$ und $\mathrm{V}_{\mathrm{i}}{ }^{0}$ liegen, obwohl die absoluten Volumenveränderungen der einzelnen Tetraeder in der Regel nicht identisch sind. Die Abbildung 42 demonstriert diese Situation für den in dieser Arbeit gefundenen Wert $\mathrm{S}=0,55$ für die Legierung Vit 4. Es sind die Volumina $\mathrm{V}_{\mathrm{i}}^{0}$ der freien Tetraeder (der reinen Komponenten) eingezeichnet. Dazu exemplarisch die mit $\mathrm{S}=0,55$ berechneten Volumina der $\mathrm{Be}_{4}$ - und $\mathrm{Zr}_{4}$-Tetraeder in der amorphen Legierung Vit 4. Die Volumina wurden entsprechend der Gleichungen (23) und (30) bestimmt und sind durch die Position der Dreiecke dargestellt. Es ist offensichtlich, dass die reinen Tetraeder der Komponenten Ni, $\mathrm{Cu}$ und Ti sehr nahe bei dem mittlerem Tetraedervolumen in der Legierung liegen. Der $\mathrm{Zr}_{4}$, aber besonders der $\mathrm{Be}_{4}$-Tetraeder liegen relativ weit davon entfernt. Dies führt dazu, dass der Wasserstoff in einem $\mathrm{Be}_{4}$-Tetraeder stärker gebunden ist, als in einem $\mathrm{Zr}_{4}$-Tetraeder, der rein chemisch betrachtet den bevorzugten Platz darstellt.

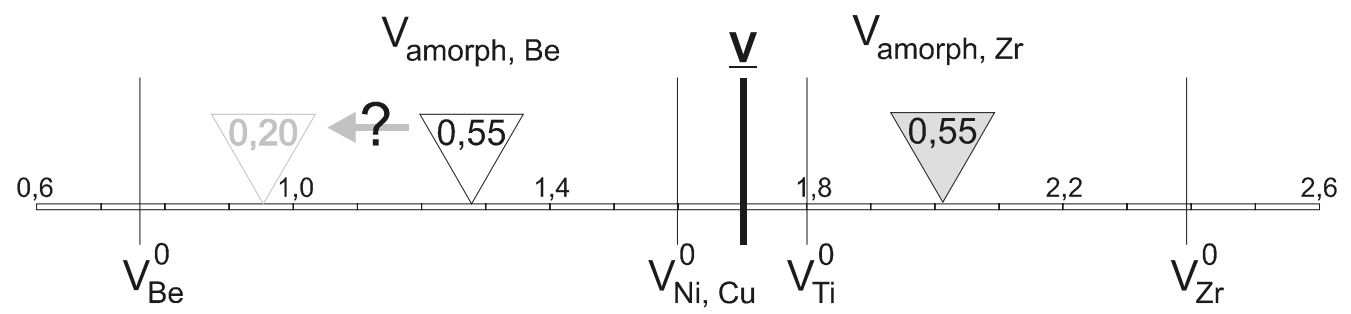

Abbildung 42: Der Einfluss des Parameters $S$ auf die Tetraedervolumina der reinen Elemente in dem konkreten Fall in der Vit 4- Legierung

Aus der Annahme eines einzigen $\mathrm{S}$-Parameters folgt somit, dass die kleineren $\mathrm{Be}_{4}$-Tetraeder in ihrer Größe wahrscheinlich sehr stark überschätzt werden. Diese übermäßige Volumenzunahme wird in dem Modell als Energiegewinn gewertet, da dieser Tetraeder nun durch den Wasserstoff viel weniger gedehnt werden muss. Die Konsequenz aus der mathematischen Beschreibung sind die erwähnten extrem niedrigen Platzenergien. Wollte man die Anpassung präziser durchführen, so ist die Einführung eines von Tetraeder zu Tetraeder unterschiedlichen Parameters $S_{i}$ sinnvoll. Jedoch hat man dann für eine fünfkomponentige Legierung nun $70 \mathrm{~S}$-Parameter mit denen experimentelle und 
theoretische Zustandsdichten angepasst werden können. Für die im Rahmen dieser Arbeit durchgeführten Berechnungen wurde daher nur der für alle Tetraeder gleiche, in Gleichung (30) eingeführte, Laufparameter S verwendet.

Des weiteren ist zu berücksichtigen, dass experimentelle Befunde [Wan98] auf eine amorphe Phasenseparation im Vitreloy hinweisen. Es liegen derzeit jedoch keine exakten Konzentrationsbestimmungen dieser Phasen vor, obwohl man davon ausgehen kann, dass sich eine $\mathrm{Zr}$ - reiche Phase neben einer $\mathrm{Be}$ - reichen Phase bildet. Unter der einfachen Annahme, dass sich die Phasenseparation nur in Be- und im Zr-Konzentrationen bemerkbar macht, also $\mathrm{Ti}$, Ni und $\mathrm{Cu}$ noch homogen über die Probe verteilt sind, ist es möglich, ebenfalls eine Anpassung der Zustandsdichte vorzunehmen. In diesem Fall kann mit zwei $\mathrm{S}$ - Parametern (einen für jede Phase) gearbeitet werden. Es wurde jedoch davon Abstand genommen, diese Interpretation und Anpassung zu vertiefen, da neben zwei S - Parametern, auch zwei Halbwertsbreiten $\sigma$ und zwei f-Werte angepasst werden mussten. Darüber hinaus können mit den Konzentrationen der fünf Legierungselemente entsprechende Anpassungsversuche durchgeführt werden. Die Anzahl der Fitparameter ist also auch in diesem Fall sehr groß. Darüber hinaus erlaubt der einfache Verlauf der gemessenen Isothermen nicht, diese Effekte mit hinreichender Genauigkeit widerzugeben. Es sollte jedoch in jedem Fall das Ziel zukünftiger Untersuchungen sein, diese Phasenseparationen, besonders in Systemen mit bekannten Zusammensetzungen der Phasen in diesem Modell zu berücksichtigen. 


\subsection{Zustandsdichten an ZANC}

In der Tabelle 8 sind die Ergebnisse aus den Anpassungen der Zustandsdichten aus der Nicht-Gleichgewichts-Isotherme (nGG) und der Gleichgewichts-Isotherme(GG) dargestellt.

Tabelle 8: Ergebnisse der Anpassung der experimentellen und theoretischen Zustandsdichten für die aus den Nicht-Gleichgewichts-Isothermen $(n G G)$ und der Gleichgewichts-Isotherme $(G G)$ gewonnenen Daten.

\begin{tabular}{|c|c|c|}
\hline & ZANC-nGG & ZANC-GG \\
\hline $\mathrm{S}$ & 0,15 & 0,1 \\
\hline$\sigma[\mathrm{kJ} / \mathrm{mol}]$ & $<3$ & 5 \\
\hline $\mathrm{f}$ & 2,5 & 3 \\
\hline
\end{tabular}

Es ist sehr interessant, dass im Gegensatz zu den Vitreloy-Proben ein viel kleiner S - Wert benötigt wird, um die experimentell ermittelten Zustandsdichten zu beschreiben. Betrachtet man noch einmal die Abbildung 5 bzw. Gleichung (30), so bedeutet dies, dass alle Tetraeder nahezu in der Größe vorliegen, wie es für freie Tetraeder zu erwarten wäre. Der Einfluss der elastischen Energiebeiträge ist daher sehr gering, da jeder einzelne Tetraeder nur minimal verzerrt vorliegt. Eventuell könnte die Probenherstellung einen Einfluss darauf haben: Durch die Sputterdeposition erzielt man sehr hohe Abschreckraten. Eine solche Probe sollte weit weg vom kristallinen Zustand sein. In dem vorgestellten Modell entspräche dies dem amorphen Zustand, in dem alle Tetraederplätze die Größe ihrer freien Äquivalente haben. Beladungsexperimente an zum Beispiel für verschieden lange Zeiten ausgelagerten aber noch immer amorphen Proben sollten darüber Aufschluss geben können. Je kleiner der S-Parameter wird, desto geringer ist auch der Einfluss der elastischen Energiebeiträge zu einer Tetraederplatzenergie. Der Vergleich mit den Ergebnissen an den Vitreloy-Legierungen zeigt, dass im Fall von ZANC dieser Einfluss geringer zu sein scheint.

Die Halbwertsbreiten wurden in der ZANC-Legierung zu Werten kleiner als $\sigma \leq 5 \mathrm{~kJ} / \mathrm{mol}$ bestimmt. Sowohl die in Vitreloy gefundenen Werte als auch Werte aus der Literatur [Kir88, Kie89] liegen in der Größenordnung $\sigma=10 \mathrm{~kJ} / \mathrm{mol}$. Für die Isothermen aus den Nicht-Gleichgewichtsmessungen gibt es einen Ansatz, der ein kleines $\sigma$ aus dem Messverfahren zusammen mit der Diffusivität des Wasserstoffes in amorphen Proben 
erklären kann: Entsprechend Gleichung (11) bzw. [Kir85] nimmt die Diffusionsgeschwindigkeit mit zunehmender Wasserstoffkonzentration zu. Da damit eine Gleichgewichtseinstellung schneller geschehen kann, bedeutet dies, dass die in diesem Versuch gemessenen Isothermen dem Gleichgewichtszustand umso näher sind, je größer die Konzentration in der Probe ist. Dies wiederum hat zur Folge, dass eine auf diese Art bestimmte Isotherme umso flacher verläuft, je mehr Wasserstoff in der Probe ist. Und je flacher eine Isotherme und ein darin enthaltenes Plateau verlaufen, desto schmaler erscheint die Halbwertsbreite des Maximums in der Zustandsdichte, das für das entsprechende Plateau verantwortlich ist. Diese Eigenschaft kann auch die im Vergleich zur berechneten Zustandsdichte sehr große Zustandsdichte (also das zweite Plateau in der Isotherme der Probe ZANC-6a in Abbildung 21) bei $\mathrm{E}=-25 \mathrm{~kJ} / \mathrm{mol}$ erklären.

Die geringe Halbwertsbreite in der Zustandsdichte die aus der Gleichgewichtsisotherme bestimmt worden ist, lässt sich nicht über diesen Ansatz erklären. Durch eine nach der Beladung durchgeführten Röntgenmessung konnte eine eventuelle Kristallisation oder strukturelle Relaxation nicht als Ursache für diese schmale Verteilung gefunden werden. Aus der Tatsache, dass es sich bei dem diskutierten Maximum um die Plätze, die durch den reinen $\mathrm{Zr}_{4}$-Tetraeder gebildet werden, handelt, könnte folgende Überlegungen eine Erklärung bieten: Die Vermutung liegt nahe, dass diese $\mathrm{Zr}_{4}$-Tetraeder versuchen, sich so anzuordnen, wie sie es aus der stöchiometrisch und strukturellen Weise im kristallinen $\mathrm{Zr}$ gewohnt sind. Zwar liegen sie noch nicht als reines Zr auskristallisiert vor, jedoch in einer elementähnlicheren dichteren Packung. Wäre dies der Fall, so kann man annehmen, dass mit Ausnahme dieser Tetraeder die anderen Tetraeder mit einer größeren Halbwertsbreite verteilt sein sollten. Dies kann die Diskrepanz der experimentellen und theoretischen Zustandsdichte in der Abbildung 34 erklären: Das beobachtete zweite und niedrigere Maximum würde durch ein größeres $\sigma$ flacher verlaufen. Eine bessere Anpassung der hier experimentell bestimmten Z(E) wäre so möglich. Die Qualität der gemessenen Isotherme und der daraus ermittelten Zustandsdichte lässt jedoch keine genaueren Aussagen zu. Zukünftige Untersuchungen sollten diesen Effekt in den ZANC-Legierungen näher studieren. Dieser Effekt kann ebenfalls die niedrigen $\sigma$-Werte in den Nicht-Gleichgewichtsmessungen erklären. Zu erwarten wäre dort somit eine Überlagerung dieses $\mathrm{Zr}_{4}$-Tetraeder-Einflusses mit dem Einfluss durch die Diffusivität wie oben beschrieben. 
Der Parameter $\mathrm{f}$ schließlich liegt mit $\mathrm{f}=2,5$ in der Nähe des zu erwartenden Wertes von $\mathrm{f}=3$. Da beim ZANC nicht die Notwendigkeit besteht, dass energetisch sehr tiefliegende Plätze in den gemessenen Bereich hinzuaddiert werden, wurde hier ein physikalisch sinnvoller Wert bestimmt. 


\subsection{Bestimmung der Wasserstoffdiffusion in amorphen Materialien}

In diesem Abschnitt werden die Ergebnisse der Durchbruchsexperimente (s. Kapitel 3.2.2), die im Kapitel 4.4 vorgestellt worden sind, näher diskutiert. Nachdem die Ursachen für die Ablösung der Schichtsysteme erörtert worden sind, wird der Einfluss der Pd-Deckschicht auf die Messergebnisse beschrieben. Schließlich wird die Druckabhängigkeit des intrinsischen Diffusionskoeffizienten von Wasserstoff in der amorphen ZANC-Legierung bei Raumtemperatur erklärt.

\subsubsection{Schichtablösung bei der Wasserstoffbeladung}

Wie die Ergebnisse in Kapitel 4.4 zeigten, wird bei zunehmenden Schichtdicken der amorphen Legierung eine schnellere Degradation der Schichtsysteme beobachtet, die auf hohe mechanische Spannungen bei der Wasserstoffbeladung hinweist. Wie hergestellt, weisen dünne Schichten in der Regel präparationsbedingte Spannungen auf [Win92]. Die Aufnahme von Wasserstoff induziert zusätzliche Spannungen [Dor02]. Vernachlässigt man den Einfluss der sehr dünnen Pd-Deckschicht, so führt zunächst der Einbau von Wasserstoff in der amorphen Matrix zu einer Volumenaufweitung [Fuk93]. Mit zunehmender Diffusionszeit erfolgt auch eine Beladung der Pd-Leitschicht, mit ebenfalls einer daraus resultierenden Volumenaufweitung. Diese Volumenaufweitungen führen zu Kräften auf die Grenzflächen zwischen Pd und Substrat, sowie zwischen amorpher Schicht und Pd, die von den Dicken der Schichten und der Wasserstoffkonzentration bzw. dem Wasserstoffkonzentrationsgradienten abhängen: Mit zunehmender Schichtdicke summieren sich näherungsweise auch die durch die Spannungen hervorgerufenen Kräfte, die auf die Schichten bzw. Grenzflächen einwirken. Daraus resultiert, dass die durch eine Aufnahme von Wasserstoff zusätzlich induzierten Spannungen und Kräfte eher zu einer Degradation des Filmes führen, als bei einer dünnen Probe, wo eine kritische Kraft erst bei höheren Wasserstoffkonzentrationen erreicht wird. Es konnte in dieser Arbeit nicht eindeutig geklärt werden, welche Grenzfläche die Degradation der Probe bestimmt. Einige Ergebnisse deuten darauf hin, dass die in der Abbildung 40 zu beobachtende makroskopische Ablösung an der Grenzfläche zwischen Substrat und Pd-Leitschicht stattfand. Ebenso können die amorphen 
und spröden Schichten bei der Volumenaufweitung Mikrorisse bilden, die eine Kurzschlussdiffusion des Wasserstoffs ermöglicht, und die Ergebnisse der eigentlichen Durchbruchszeiten verfälscht, was zu der unphysikalischen Abnahme der Durchbruchszeiten mit zunehmender Schichtdicke in Abbildung 38 führt. Ob und in wieweit die Bildung von Mikrorissen durch eine geeignete Wahl des Substrates beeinflusst werden kann, erfordert ein besseres Verständnis der rissbildenden Mechanismen. Unabhängig davon muss man davon ausgehen, dass für die Messungen der Durchbruchszeiten eine Wasserstoffkonzentrationsabhängige kritische Schichtdicke der amorphen Schicht existiert. Unterhalb dieser Schichtdicke ist der Widerstandsverlauf reversibel und lässt eine reproduzierbare Bestimmung der Durchbruchszeiten zu.

\subsubsection{Einfluss der Pd-Deckschicht auf den gemessenen Widerstand}

In der Abbildung 37 und der Abbildung 39 ist kein Einfluss der ca. $8 \mathrm{~nm}$ dicken Pd-Deckschichten auf die Widerstandsänderung zu sehen. Es wäre zu erwarten, dass durch die Wasserstoffbeladung und der damit verbundenen Widerstandsänderung der Pd-Deckschicht eine Stufe im Widerstandsverlauf zu Beginn einer jeden Beladung zu beobachten ist. Das Fehlen dieses Einflusses folgt aus den bei einer nominellen Schichtdicke von $8 \mathrm{~nm}$ noch nicht geschlossenen, aus Inseln bestehende Pd Bedeckung [Dan02]. Bei nominell nur ca. 2,5 nm dickeren Pd-Deckschichten konnte eine Abnahme des Widerstandes bei Wasserstoffaufnahme als Folge von Perkolation dieser Inseln beobachtet werden. Zwei Durchbruchszeitenmessungen die diesen Effekt zeigen sind in Abbildung 43 dargestellt. 


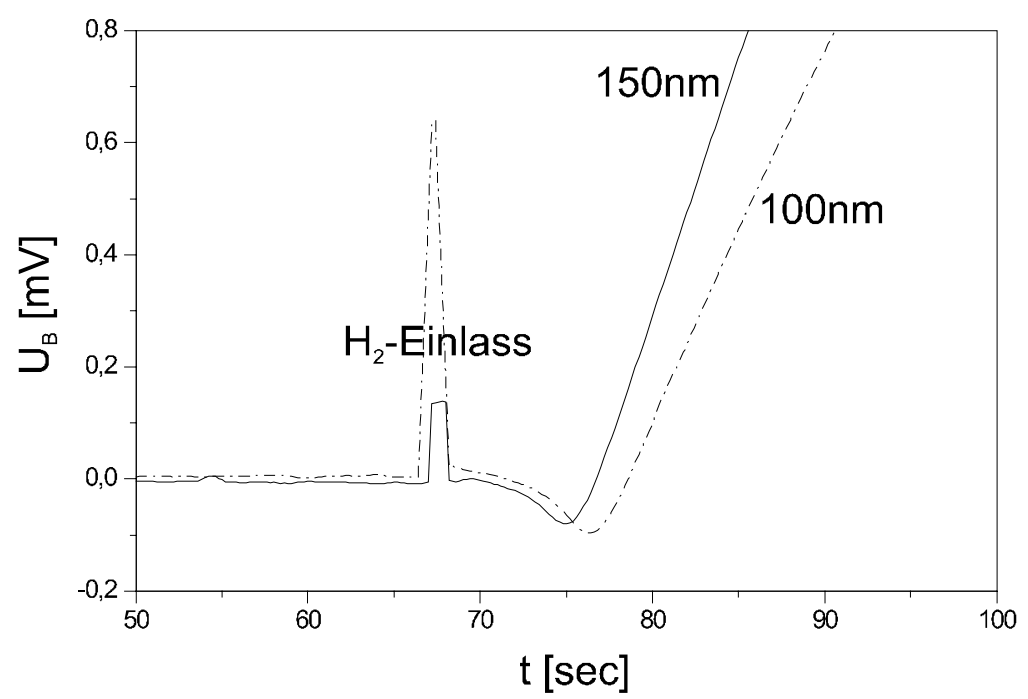

Abbildung 43: Durchbruchsmessung an zwei Proben - das Minimum folgt aus der Perkolation sich ausdehnender Pd-Inseln. Diese bilden die nicht geschlossene Pd-Deckschicht.

In [Dan02] wurde gezeigt, dass ca. 10nm dicke Pd-Schichten noch nicht geschlossen sind und aus voneinander isolierten Inseln bestehen. Somit liegt an solchen Proben keine elektrische Leitfähigkeit vor. Bei Beladung der Pd-Inseln mit Wasserstoff dehnen sich diese gemäß [Dan02, Fuk93] aus. Waren die Inseln ursprünglich dicht genug beieinander, so führt diese Ausdehnung $\mathrm{zu}$ einer Perkolation sich berührender Inseln über die gesamte Probenbreite. Mit der so erhaltenen elektrischen Leitfähigkeit dieser Schicht folgt auch ein Beitrag zum Gesamtwiderstand der Probe. Da offensichtlich nominell nur 2,5 nm bis zur Perkolationsfähigkeit „fehlen“, kann man von einer großflächigen Bedeckung der Oberfläche bei den untersuchten Proben ausgehen. Somit ist der Zweck der Pd-Deckschicht, die amorphe Schicht vor Oxidation zu schützen und die Wasserstoffaufnahme zu katalysieren, jedoch ohne einen Einfluss auf die Widerstandsmessungen zu haben, gegeben.

\subsubsection{Bestimmung des intrinsischen Diffusionskoeffizienten von Wasserstoff in amorphen $\mathrm{Zr}_{66,8} \mathrm{Al}_{17,4} \mathrm{Ni}_{7,2} \mathrm{Cu}_{8,8}(\mathrm{ZANC})$ - Schichten}

In Kapitel 2.2 wurde die Gleichung (15) hergeleitet, die eine wurzelförmige Abhängigkeit des intrinsischen Wasserstoffdiffusionskoeffizienten vom Wasserstoffpartialdruck 
beschreibt, falls $\delta \mu_{\mathrm{H}} / \delta \mathrm{c}_{\mathrm{H}}$ nicht von der Wasserstoffkonzentration abhängt. Nach Gleichung (4) ist diese Ableitung gleich der reziproken effektiven Zustandsdichte $Z(\mu)$. In dem Druckbereich von $10^{-5}$ bar $\left(\mu_{\mathrm{H}} \approx-5 \mathrm{~kJ} / \mathrm{mol}\right)$ bis $10^{-2}$ bar $\left(\mu_{\mathrm{H}} \approx-14 \mathrm{~kJ} / \mathrm{mol}\right)$, in $\operatorname{dem} \operatorname{der}$ Diffusionkoeffizient gemessen wurde, ist $Z(\mu)$ praktisch nicht von $\mu_{\mathrm{H}}$ (s. Abbildung 34) und somit auch nicht von der Wasserstoffkonzentration abhängig.

Um die in Gleichung (15) vorhergesagte Abhängigkeit von $\left(\mathrm{p}_{\mathrm{H} 2}\right)^{1 / 2}$ zu überprüfen, sind in der Abbildung 36 die Werte in der Form $1 / \Delta \mathrm{t}$ als Funktion von $\left(\mathrm{p}_{\mathrm{H} 2} / \mathrm{p}_{\mathrm{H} 2,0}\right)^{1 / 2}$ doppelllogarithmisch aufgetragen worden. In dieser Auftragung ist entsprechend Gleichung (15) ein linearer Zusammenhang der Messgrößen mit der Steigung 1 zu erwarten.

Die Abbildung 36 zeigt tatsächlich eine gute Übereinstimmung von Theorie und Experiment. Die Messwerte sind durchaus mit dem zu erwartenden linearen Verhalten vereinbar. Mit Gleichung (40), der Schichtdicke $\mathrm{s}=50 \mathrm{~nm}=5 \times 10^{-6} \mathrm{~cm}$ und dem Wert $1 / \Delta \mathrm{t} \approx 0,06$ an der Stelle $\log \left(\mathrm{p}_{\mathrm{H} 2} / \mathrm{p}_{\mathrm{H} 2,0}\right)=0$ erhält man für den experimentell ermittelten intrinsischen Diffusionskoeffizienten entleerter ZANC-Proben bei Raumtemperatur folgenden Zusammenhang mit dem Wasserstoffdruck:

$$
D_{i n} \approx 2,5 \cdot 10^{-13} \frac{\mathrm{cm}^{2}}{\mathrm{sec}} \cdot \sqrt{\frac{p_{H 2}}{p_{H 2,0}}} ; \quad\left(\text { mit } p_{H 2,0}=1 \mathrm{mbar}\right)
$$

Aus den Abbildungen 4.6 und 4.8 aus der Referenz [Kie89] erhält man bei Raumtemperatur für die Diffusionskoeffizienten für die amorphen Legierungen $\mathrm{Ni}_{35} \mathrm{Zr}_{65}$ und $\mathrm{Ni}_{65} \mathrm{Zr}_{35}$ Werte im Bereich $10^{-9} \mathrm{~cm}^{2} / \mathrm{sec}$ bis $10^{-7} \mathrm{~cm}^{2} / \mathrm{sec}$ bei Wasserstoffkonzentrationen um $\mathrm{c}_{\mathrm{H}} \approx 0,5 \mathrm{H} / \mathrm{Me}$. Bei einem Druck von 0,01 mbar folgt nach Gleichung (37) mit $\mathrm{U}_{0}=-182 \mathrm{mV}$ bei gleichem chemischen Potential für die EMK: $U \approx-40 \mathrm{mV}$. Aus der Gleichgewichtsisotherme in Abbildung 21 lässt sich dann eine Konzentration von $\mathrm{c}_{\mathrm{H}} \approx 0,6 \mathrm{H} / \mathrm{Me}$ in der Probe bestimmen. Aus Gleichung (41) folgt dann: $D_{\text {in }} \approx 2,510^{-14} \mathrm{~cm}^{2} / \mathrm{sec}$. Der für die ZANCLegierung bestimmte Wert ist also um mindestens vier bis fünf Größenordnungen kleiner als in den erwähnten NiZr-Legierungen. 
Es sei noch erwähnt, dass entsprechend Gleichung (11) eigentlich eine nicht zu vernachlässigende (1-c) $)^{2}$-Abhängigkeit des Diffusionskoeffizienten zu erwarten ist. Die physikalische Ursache für eine Zunahme des Diffusionskoeffizienten liegt in dem Auffüllen von $Z(E)$ mit zunehmendem chemischen Potential $\mu_{\mathrm{H}}$ : Je mehr Wasserstoff gelöst ist, desto mehr energetisch tiefe Plätze sind besetzt und umso größer ist die Energie der Plätze, die durch zusätzliche Atome besetzt werden können. Die Energiedifferenz eines Platzes bei dem chemischen Potential $\mu_{\mathrm{H}} \mathrm{zu}$ einer bestimmten Sattelpunktsenergie $\mathrm{G}^{0}$ wird also umso geringer, je höher die Konzentration an Wasserstoff in der Probe ist. Je kleiner diese Energiedifferenz $\left(\mu_{\mathrm{H}^{-}} \mathrm{G}^{0}\right)$, die der Aktivierungsenergie für die Diffusion entspricht, ist, desto größer wird nach Gleichungen (11) und (13) der Diffusionskoeffizient [Kir88].

Diese Konzentrationsabhängigkeit ist der Grund für die Streuung der Messwerte in Abbildung 36. Vor der Bestimmung der einzelnen Durchbruchszeiten wurden die Proben entleert, indem der Rezipient für einige Zeit abgepumpt worden ist. Es kann leider nicht ausgeschlossen werden, dass zu Beginn eines jeden Experimentes noch eine nicht genau bestimmbare Ausgangskonzentration in den Proben vorlag, da die Abpumpzeiten zwischen einigen Stunden und einigen Tagen variierten. Der Einfluss dieser Zeiten wurde erst bei der Auswertung der gewonnenen Daten offensichtlich. Zukünftige Untersuchungen sollten zum Ziel haben, mit noch genauerer Sicherheit die Diffusionskoeffizientenbestimmung durchzuführen. 


\section{Zusammenfassung}

In dieser Arbeit wurde ein Modell zur Beschreibung von Wasserstoff in amorphen binären Legierungen erweitert, um auch mehrkomponentige Legierungen hinsichtlich ihres Wasserstofflösungsverhaltens zu beschreiben. Dabei wird von einer Energieverteilung der Zwischengitterplätze (Tetraeder) im Sinne einer Zustandsdichte für den Wasserstoff ausgegangen. Die Energie eines statistisch möglichen in einer Legierung vorkommenden Tetraedertyps wird nach Berücksichtigung chemischer Lösungsenergien durch einen Beitrag einer elastischen Verzerrungsenergie individuell in Abhängigkeit der Tetraederzusammensetzung für jeden Tetraederplatztyp berechnet. Die Einflüsse der elastischen Energiebeiträge sind besonders bei unterschiedlichen Atomradien der Legierungskomponenten sehr groß und können die chemisch zu erwartenden Lösungsenergien stark verändern.

Am Beispiel zweier Vitreloy-Legierungen und einer ZANC-Legierung $\left(\mathrm{Zr}_{66,8} \mathrm{Al}_{17,4} \mathrm{Ni}_{7,2} \mathrm{Cu}_{8,8}\right)$, die als Prototypen für massive metallische Gläser verwendet wurden, konnten experimentell bestimmte Energieverteilungen mit den berechneten verglichen werden. Die zur Anpassung der Kurven verwendeten Parameter S (berücksichtigt das Tetraedervolumen), $\sigma$ (entspricht der Energieverteilung jedes Tetraeders) und $f$ (entspricht der Zahl der Tetraeder, die mathematisch gesehen von einem Atom gebildet werden) konnten physikalisch sinnvoll gewählt werden. Damit hat man einen Hinweis auf die Gültigkeit der gemachten Annahmen gewonnen.

Neben dem Wasserstofflösungsverhalten kann mit Hilfe dieser Energieverteilung auch das Diffusionsverhalten von Wasserstoff in amorphen mehrkomponentigen Legierungen berechnet werden. Um zukünftig die berechneten Diffusionskoeffizienten mit experimentellen Daten vergleichen zu können wurde eine neue Methode entwickelt, die es erlaubt, quantitative Diffusionsmessungen durchzuführen. Herkömmliche Methoden vcrsagen an den untersuchten Legierungen, da deren Sprödigkeit stets eine Zerstörung der Probe bei Wasserstoffbeladung zur Folge hat. 
Bei der neu entwickelten Methode wird die Probe in Form eines dünnen Filmes auf einer Pd-Leitschicht präpariert. Der an der Probenoberfläche angebotene Wasserstoff diffundiert dann durch die zu untersuchende Schicht in die Pd-Leitschicht und wird dort über eine Widerstandsänderung detektiert.

Dieses Prinzip entspricht einem Permeationsexperiment mit dessen Hilfe Durchbruchszeiten bestimmt werden können, um daraus Wasserstoffdiffusionskoeffizienten $\mathrm{zu}$ berechnen. Gegenüber herkömmlichen Permeationsmessungen konnte bei der neuen Methode die Probendicke soweit reduziert werden, dass bei der Sorption von Wasserstoff keine Risse und damit keine Kurzschlussdiffusion mehr auftrat.

Es wurden schließlich mit diesem Verfahren Durchbruchszeiten an einer ZANC - Legierung bestimmt, wodurch zum ersten mal in der Literatur die quantitative Bestimmung des intrinsischen Wasserstoffdiffusionskoeffizienten in massiven metallischen Gläsern als Funktion des Druckes bei Raumtemperatur bestimmt werden konnte. Dieser ist erstaunlicher Weise vier bis fünf Größenordnungen niedriger als z.B. in NiZr-Legierungen. Mit dieser Methode hat man für zukünftige Messungen die Voraussetzung geschaffen, quantitative Untersuchungen des Diffusionsverhaltens von Wasserstoff auch in Abhängigkeit der Temperatur oder der Wasserstoffkonzentration durchzuführen. 


\section{ANHANG}

\subsection{Phasendiagramm Pd-H}

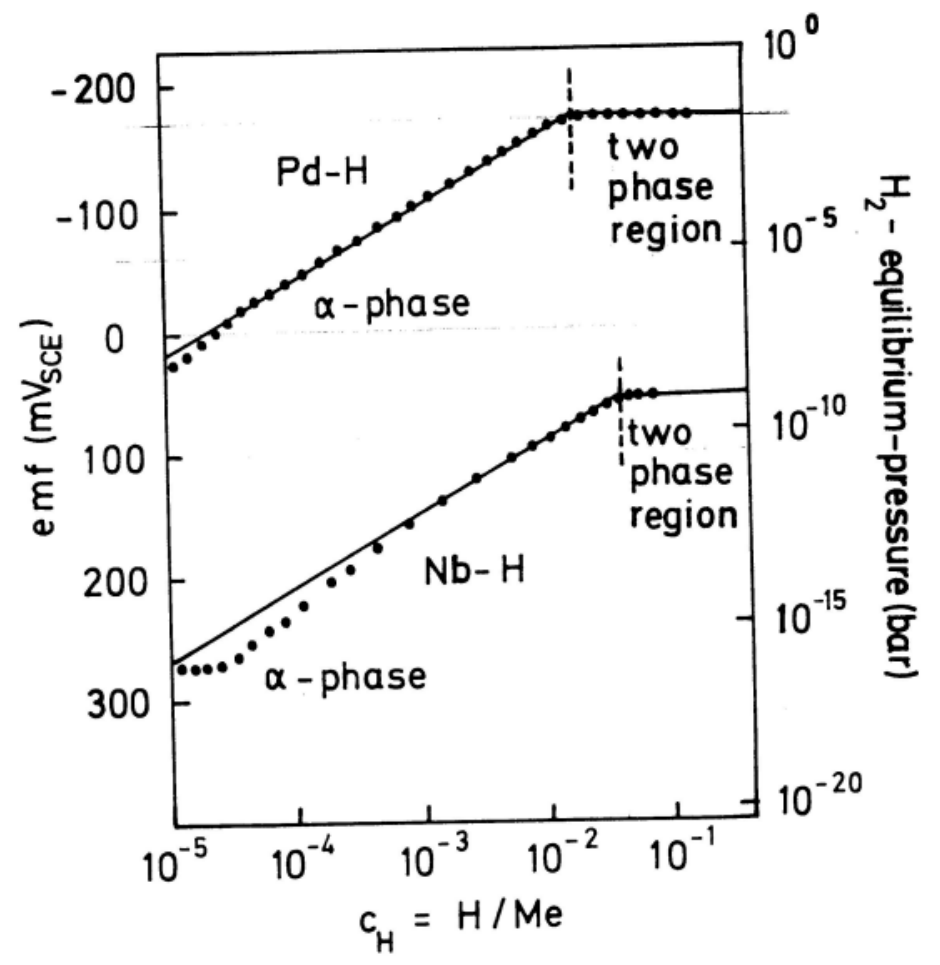

Abbildung 44: Phasendiagramme von Pd-H und Nb-H aus [Kir88] für kleine Wasserstoffkonzentrationen. Die EMK (engl.: emf - electro motoric force) wurde gegen eine $\mathrm{Hg} / \mathrm{HgCl}-$ Elektrode mit $U_{0}=-220 \mathrm{mV}$ bestimmt. In dieser Arbeit betrug $U_{0}=-182 \mathrm{mV}$, da Ag/AgCl-Elektroden verwendet wurden. Die EMK-Skala muss in dieser Darstellung dann um $38 \mathrm{mV}$,,nach oben “verschoben werden. Das Zwei-Phasengebiet für Pd-PdH liegt dann bei $U \approx-135 \mathrm{mV}$. 


\subsection{Wheatstonesche Brücke}

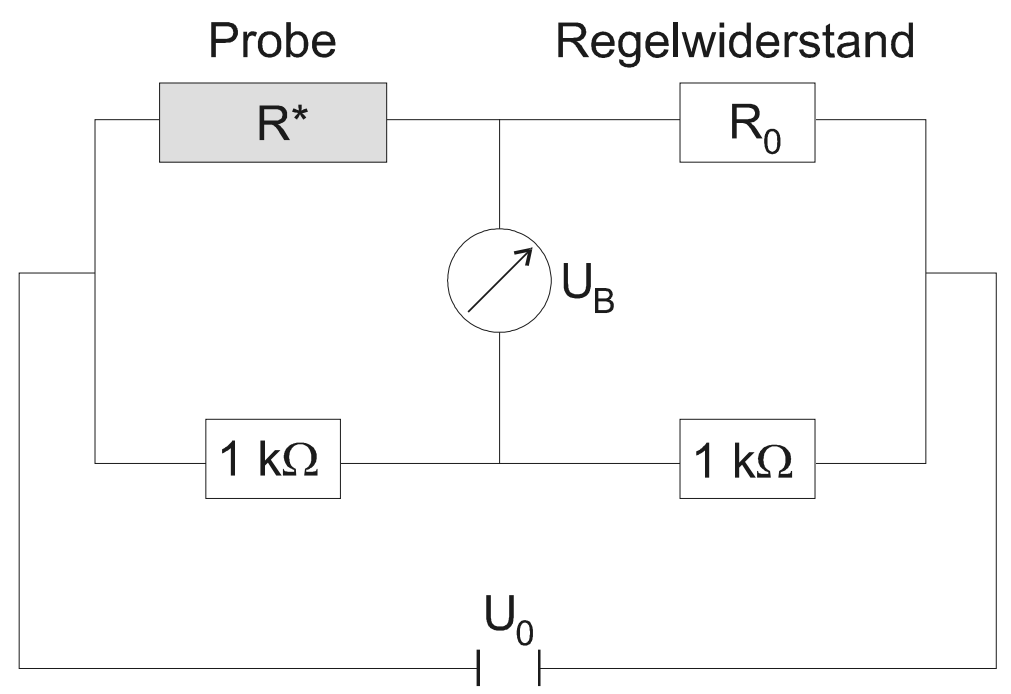

Abbildung 45: Wheatstonesche Brückenschaltung

Die Wheatstonsche Brückenschaltung besteht aus vier Widerständen, die entsprechend Abbildung 45 miteinander verschaltet sind. An der Brücke liegt die Speisespannung $\mathrm{U}_{0}$ an. Wenn der Probenwiderstand $\mathrm{R}^{*}$ genauso groß ist, wie der Regelwiderstand $\mathrm{R}_{0}$, beträgt die abgreifbare Brückenspannung $U_{B}=0 \mathrm{~V}$. Bei einer Veränderung des Probenwiderstandes ändert sich die Brückenspannung gemäß [Ger93]:

$$
U_{B}=\frac{1}{4} \cdot U_{0} \cdot \frac{R^{*}-R_{0}}{R_{0}}
$$

Das entscheidende an diesem Aufbau ist, dass die Änderung des Widerstandes $\mathrm{R}^{*}$ und nicht sein Absolutwert gemessen wird. Damit hat man bei geeigneter Wahl von $\mathrm{U}_{0}$ die Möglichkeit, eine Änderung von $\mathrm{R}^{*}$ sehr empfindlich nachzuweisen. Für die relative Widerstandsänderung folgt:

$$
\frac{R^{*}}{R_{0}}=4 \cdot \frac{U_{B}}{U_{0}}+1
$$




\subsection{Die Tetraeder in Vit 1}

Folgende 70 Tetraeder sind in einer $\mathrm{Zr}_{41} \mathrm{Ti}_{14} \mathrm{Cu}_{12.5} \mathrm{Ni}_{10} \mathrm{Be}_{22.5}$-Legierung (Vit 1) möglich. Die Wahrscheinlichkeit einen bestimmten Tetraeder in der Legierung $\mathrm{zu}$ finden, $\mathrm{W}_{\mathrm{i}}$ (s. Kapitel 2.3.1), und die mittlere Energie $E_{i}$ sind sowohl mit, als auch ohne den Beitrag elastischer Energien berechnet worden (s. Kapitel 2.3.2). Der Einfluss des Wasserstoffblockings (s. Kapitel 2.3.3) ist bereits berücksichtigt. Die 15 häufigsten Tetraeder sind mit einem Stern gekennzeichnet. Die Tetraeder sind aufsteigend nach der mittleren Energie nach Berücksichtigung der elastischen Energien für $\mathrm{S}=0,55$ sortiert.

Tetraeder (Vit 1) $\quad W_{\mathrm{i}}$ mit El. E $_{\mathrm{i}}$ mit El. $[\mathrm{kJ} / \mathrm{mol}] \mathrm{W}_{\mathrm{i}}$ ohne El. E i $_{\mathrm{i}}$ ohne El. [kJ/mol]

\begin{tabular}{|c|c|c|c|c|}
\hline $\mathrm{Be} 4$ & 0.0026 & -529.04 & 0.0026 & -1.84 \\
\hline Ni1Be3 & 0.0046 & -363.40 & 0.0046 & 2.79 \\
\hline $\mathrm{Cu} 1 \mathrm{Be} 3$ & 0.0057 & -342.30 & 0.0057 & 10.88 \\
\hline Ti1Be3 & 0.0064 & -270.10 & 0.0064 & -12.69 \\
\hline $\mathrm{Ni} 2 \mathrm{Be} 2$ & 0.0030 & -257.55 & 0.0030 & 7.42 \\
\hline Ni1Cu1Be2 & 0.0076 & -239.33 & 0.0076 & 15.51 \\
\hline $\mathrm{Cu} 2 \mathrm{Be} 2$ & 0.0047 & -221.24 & 0.0047 & 23.60 \\
\hline Ti1Ni1Be2 & 0.0085 & -190.56 & 0.0085 & -8.06 \\
\hline Ni3Be1 & 0.0009 & -184.04 & 0.0009 & 12.05 \\
\hline $\mathrm{Zr} 1 \mathrm{Be} 3$ & 0.0187 & -178.59 & 0.0187 & -16.26 \\
\hline TilCu1Be2 & 0.0106 & -173.73 & 0.0106 & 0.03 \\
\hline Ni2Cu1Be1 & 0.0034 & -167.77 & 0.0034 & 20.14 \\
\hline Ni1Cu2Be1 & 0.0042 & -151.61 & 0.0042 & 28.23 \\
\hline Ti2Be 2 & 0.0060 & -136.69 & 0.0060 & -23.54 \\
\hline $\mathrm{Cu} 3 \mathrm{Be} 1$ & 0.0018 & -135.57 & 0.0018 & 36.32 \\
\hline Ti1Ni2Be1 & 0.0038 & -134.33 & 0.0038 & -3.43 \\
\hline $\mathrm{Ni} 4$ & 0.0001 & -129.93 & 0.0001 & 16.68 \\
\hline Zr1Ni1Be2 & 0.0249 & -124.57 & 0.0249 & -11.63 \\
\hline Ti1Ni1Cu1Be1 & 0.0095 & -119.12 & 0.0095 & 4.66 \\
\hline $\mathrm{Ni3Cul}$ & 0.0005 & -115.08 & 0.0005 & 24.77 \\
\hline
\end{tabular}


Tetraeder (Vit 1) $\quad W_{\text {i }}$ mit El. $E_{i}$ mit El. [kJ/mol] $W_{i}$ ohne El. E $E_{i}$ ohne El. [kJ/mol]

\begin{tabular}{|c|c|c|c|c|c|}
\hline Zr1Cu1Be2 & * (11) & 0.0311 & -109.40 & 0.0311 & -3.54 \\
\hline Ti1Cu2Be1 & & 0.0059 & -104.02 & 0.0059 & 12.75 \\
\hline $\mathrm{Ni} 2 \mathrm{Cu} 2$ & & 0.0009 & -100.31 & 0.0009 & 32.86 \\
\hline Ti2Ni1Be1 & & 0.0053 & -94.37 & 0.0053 & -18.91 \\
\hline Ti1Ni3 & & 0.0006 & -92.34 & 0.0006 & 1.20 \\
\hline Ni1Cu3 & & 0.0008 & -85.64 & 0.0008 & 40.95 \\
\hline Zr1Ti1Be2 & $*(9)$ & 0.0349 & -85.28 & 0.0349 & -27.11 \\
\hline Zr1Ni2Be1 & & 0.0111 & -84.62 & 0.0111 & -7.00 \\
\hline Ti2Cu1Be1 & & 0.0066 & -80.05 & 0.0066 & -10.82 \\
\hline Ti1Ni2Cu1 & & 0.0021 & -78.33 & 0.0021 & 9.29 \\
\hline $\mathrm{Cu} 4$ & & 0.0002 & -71.05 & 0.0002 & 49.04 \\
\hline Zr1Ni1Cu1Be1 & $*(15)$ & 0.0277 & -70.65 & 0.0277 & 1.09 \\
\hline Ti1Ni1Cu2 & & 0.0026 & -64.39 & 0.0026 & 17.38 \\
\hline Ti3Be1 & & 0.0025 & -62.49 & 0.0025 & -34.39 \\
\hline Ti2Ni2 & & 0.0012 & -62.29 & 0.0012 & -14.28 \\
\hline $\mathrm{Zr} 1 \mathrm{Cu} 2 \mathrm{Be} 1$ & & 0.0173 & -56.76 & 0.0173 & 9.18 \\
\hline Zr1Ti1NilBe1 & * (12) & 0.0310 & -55.29 & 0.0310 & -22.48 \\
\hline Zr1Ni3 & & 0.0016 & -53.73 & 0.0016 & -2.37 \\
\hline Ti1Cu3 & & 0.0011 & -50.53 & 0.0011 & 25.47 \\
\hline Ti2Ni1Cu1 & & 0.0029 & -48.99 & 0.0029 & -6.19 \\
\hline $\mathrm{Zr} 2 \mathrm{Be} 2$ & $*(4)$ & 0.0511 & -47.93 & 0.0511 & -30.68 \\
\hline Zr1Ti1Cu1Be1 & $*(6)$ & 0.0387 & -42.02 & 0.0387 & -14.39 \\
\hline $\mathrm{Zr} 1 \mathrm{Ni} 2 \mathrm{Cu} 1$ & & 0.0062 & -40.68 & 0.0062 & 5.7 \\
\hline Ti3Ni1 & & 0.0011 & -38.56 & 0.0011 & -29.76 \\
\hline $\mathrm{Ti} 2 \mathrm{Cu} 2$ & & 0.0018 & -35.75 & 0.0018 & 1.90 \\
\hline Zr1Ti2Be1 & & 0.0217 & -32.21 & 0.0217 & -37.96 \\
\hline Zr1Ti1Ni2 & & 0.0069 & -31.77 & 0.0069 & -17.85 \\
\hline Zr1Ni1Cu2 & & 0.0077 & -27.69 & 0.0077 & 13.81 \\
\hline Zr2Ni1Be1 & $*(5)$ & 0.0454 & -26.27 & 0.0454 & -26.05 \\
\hline Ti3Cu1 & & 0.0014 & -25.85 & 0.0014 & -21.67 \\
\hline Ti4 & & 0.0004 & -20.15 & 0.0004 & -45.24 \\
\hline Zr1TilNi1Cu1 & & 0.0172 & -19.28 & 0.0172 & -9.76 \\
\hline
\end{tabular}


Tetraeder (Vit 1) $\quad$ W $_{\mathrm{i}}$ mit El. E $_{\mathrm{i}}$ mit El. [kJ/mol] $\mathbf{W}_{\mathrm{i}}$ ohne El. E Ehe $_{\mathbf{i}}$ ohne El. [kJ/mol]

\begin{tabular}{cccccc}
\hline \hline Zr1Ti2Ni1 & & 0.0096 & -14.78 & 0.0096 & -33.33 \\
Zr1Cu3 & & 0.0032 & -14.75 & 0.0032 & 21.90 \\
Zr2Cu1Be1 & $*(3)$ & 0.0567 & -13.82 & 0.0567 & -17.96 \\
Zr2Ti1Be1 & $*(1)$ & 0.0635 & -9.90 & 0.0635 & -41.53 \\
Zr2Ni2 & & 0.0101 & -8.75 & 0.0101 & -21.42 \\
Zr1Ti1Cu2 & & 0.0108 & -6.84 & 0.0108 & -1.67 \\
Zr1Ti2Cu1 & & 0.0121 & -2.78 & 0.0121 & -25.24 \\
Zr1Ti3 & & 0.0045 & -2.04 & 0.0045 & -48.81 \\
Zr2Ti1Ni1 & $*(14)$ & 0.0282 & 2.95 & 0.0282 & -36.90 \\
Zr2Ni1Cu1 & & 0.0252 & 3.08 & 0.0252 & -13.33 \\
Zr3Be1 & $*(2)$ & 0.0620 & 6.54 & 0.0620 & -45.10 \\
Zr2Ti2 & & 0.0198 & 11.23 & 0.0198 & -52.38 \\
Zr2Ti1Cu1 & $*(8)$ & 0.0353 & 14.39 & 0.0353 & -28.81 \\
Zr2Cu2 & & 0.0158 & 14.88 & 0.0158 & -5.24 \\
Zr3Ni1 & & 0.0276 & 16.12 & 0.0276 & -40.47 \\
Zr3Ti1 & $*(7)$ & 0.0386 & 20.84 & 0.0386 & -55.95 \\
Zr3Cu1 & $*(10)$ & 0.0345 & 27.12 & 0.0345 & -32.38 \\
Zr4 & $*(13)$ & 0.0283 & 27.63 & 0.0283 & -59.52 \\
& & & & &
\end{tabular}




\subsection{Die Tetraeder in Vit 4}

Folgende Tetraeder sind in einer $\mathrm{Zr}_{46.75} \mathrm{Ti}_{8.25} \mathrm{Cu}_{7.5} \mathrm{Ni}_{10} \mathrm{Be}_{27.5}$-Legierung möglich. Die Wahrscheinlichkeit einen bestimmten Tetraeder in der Legierung $\mathrm{zu}$ finden $\mathrm{W}_{\mathrm{i}}$ (s. Kapitel 2.3.1) und die mittlere Energie $E_{i}$ sind sowohl mit, als auch ohne den Beitrag elastischer Energien berechnet worden (s. Kapitel 2.3.2). Der Einfluss des Wasserstoffblockings (s. Kapitel 2.3.3) ist bereits berücksichtigt. Die 15 häufigsten Tetraeder sind mit einem Stern gekennzeichnet. Die Tetraeder sind aufsteigend nach der mittleren Energie nach Berücksichtigung der elastischen Energien für $\mathrm{S}=0,55$ sortiert.

Tetraeder (Vit 4) $\quad$ W $_{\mathrm{i}}$ mit El. E $_{\mathrm{i}}$ mit El. [kJ/mol] $\mathbf{W}_{\mathrm{i}}$ ohne El. E $_{\mathrm{i}}$ ohne El. [kJ/mol]

\begin{tabular}{|c|c|c|c|c|c|}
\hline $\mathrm{Be} 4$ & & 0.005719 & -534.90 & 0.005719 & -1.84 \\
\hline Ni1Be3 & & 0.008319 & -368.13 & 0.008319 & 2.79 \\
\hline Cu1Be3 & & 0.006239 & -347.08 & 0.006239 & 10.88 \\
\hline Ti1Be3 & & 0.006863 & -274.91 & 0.006863 & -12.69 \\
\hline $\mathrm{Ni} 2 \mathrm{Be} 2$ & & 0.004538 & -261.55 & 0.004538 & 7.42 \\
\hline Ni1Cu1Be2 & & 0.006806 & -243.35 & 0.006806 & 15.51 \\
\hline $\mathrm{Cu} 2 \mathrm{Be} 2$ & & 0.002552 & -225.30 & 0.002552 & 23.6 \\
\hline Ti1Ni1Be2 & & 0.007487 & -194.61 & 0.007487 & -8.06 \\
\hline Ni3Bel & & 0.0011 & -187.51 & 0.0011 & 12.05 \\
\hline Zr1Be3 & $*(9)$ & 0.038932 & -182.90 & 0.038932 & -16.26 \\
\hline TilCu1Be2 & & 0.005615 & -177.81 & 0.005615 & 0.03 \\
\hline Ni2Cu1Be1 & & 0.002475 & -171.26 & 0.002475 & 20.14 \\
\hline $\mathrm{Ni} 1 \mathrm{Cu} 2 \mathrm{Be} 1$ & & 0.001856 & -155.13 & 0.001856 & 28.23 \\
\hline Ti2Be 2 & & 0.003088 & -140.74 & 0.003088 & -23.54 \\
\hline $\mathrm{Cu} 3 \mathrm{Be} 1$ & & 0.000464 & -139.10 & 0.000464 & 36.32 \\
\hline Ti1Ni2Be1 & & 0.002723 & -137.84 & 0.002723 & -3.43 \\
\hline $\mathrm{Ni4}$ & & 0.0001 & -133.00 & 0.0001 & 16.68 \\
\hline Zr1Ni1Be2 & $*(7)$ & 0.042471 & -128.27 & 0.042471 & -11.63 \\
\hline Ti1Ni1Cu1Be1 & & 0.004084 & -122.65 & 0.004084 & 4.66 \\
\hline Ni3Cu1 & & 0.0003 & -118.17 & 0.0003 & 24.77 \\
\hline
\end{tabular}


Tetraeder (Vit 4) $\quad$ W $_{\text {i }}$ mit El. E E mit El. [kJ/mol] $W_{i}$ ohne El. $E_{i}$ ohne El. [kJ/mol]

\begin{tabular}{|c|c|c|c|c|c|}
\hline Zr1Cu1Be2 & *(12) & 0.031853 & -113.11 & 0.031853 & -3.54 \\
\hline Ti1Cu2Be1 & & 0.001531 & -107.56 & 0.001531 & 12.75 \\
\hline $\mathrm{Ni2Cu} 2$ & & 0.000338 & -103.42 & 0.000338 & 32.86 \\
\hline Ti2Ni1Be1 & & 0.002246 & -97.87 & 0.002246 & -18.91 \\
\hline Ti1Ni3 & & 0.00033 & -95.44 & 0.00033 & 1.2 \\
\hline Zr1Ti1Be2 & $*(10)$ & 0.035039 & -88.95 & 0.035039 & -27.11 \\
\hline Ni1Cu3 & & 0.000169 & -88.76 & 0.000169 & 40.95 \\
\hline Zr1Ni2Be1 & & 0.015444 & -87.86 & 0.015444 & -7 \\
\hline Ti2Cu1Be1 & & 0.001685 & -83.57 & 0.001685 & -10.82 \\
\hline Ti1Ni2Cul & & 0.000743 & -81.44 & 0.000743 & 9.29 \\
\hline $\mathrm{Cu} 4$ & & 0.000032 & -74.19 & 0.000032 & 49.04 \\
\hline Zr1Ni1Cu1Be1 & $*(15)$ & 0.023166 & -73.91 & 0.023166 & 1.09 \\
\hline Ti1Ni1Cu2 & & 0.000557 & -67.52 & 0.000557 & 17.38 \\
\hline Ti3Be1 & & 0.000618 & -65.96 & 0.000618 & -34.39 \\
\hline $\mathrm{Ti} 2 \mathrm{Ni} 2$ & & 0.000408 & -65.39 & 0.000408 & -14.28 \\
\hline $\mathrm{Zr} 1 \mathrm{Cu} 2 \mathrm{Be} 1$ & & 0.008687 & -60.03 & 0.008687 & 9.18 \\
\hline Zr1Ti1Ni1Be1 & $*(14)$ & 0.025483 & -58.51 & 0.025483 & -22.48 \\
\hline Zr1Ni3 & & 0.001872 & -56.62 & 0.001872 & -2.37 \\
\hline Ti1Cu3 & & 0.000139 & -53.67 & 0.000139 & 25.47 \\
\hline Ti2Ni1Cu1 & & 0.000613 & -52.09 & 0.000613 & -6.19 \\
\hline $\mathrm{Zr} 2 \mathrm{Be} 2$ & $*(2)$ & 0.099382 & -51.28 & 0.099382 & -30.68 \\
\hline Zr1Ti1Cu1Be1 & & 0.019112 & -45.25 & 0.019112 & -14.39 \\
\hline $\mathrm{Zr} 1 \mathrm{Ni} 2 \mathrm{Cu} 1$ & & 0.004212 & -43.58 & 0.004212 & 5.72 \\
\hline Ti3Ni1 & & 0.000225 & -41.62 & 0.000225 & -29.76 \\
\hline $\mathrm{Ti} 2 \mathrm{Cu} 2$ & & 0.00023 & -38.86 & 0.00023 & 1.9 \\
\hline Zr1Ti2Be1 & & 0.010512 & -35.38 & 0.010512 & -37.96 \\
\hline Zr1Ti1Ni2 & & 0.004633 & -34.63 & 0.004633 & -17.85 \\
\hline Zr1Ni1Cu2 & & 0.003159 & -30.59 & 0.003159 & 13.81 \\
\hline Zr2Ni1Be1 & *(3) & 0.072278 & -29.24 & 0.072278 & -26.05 \\
\hline Ti3Cul & & 0.000168 & -28.92 & 0.000168 & -21.67 \\
\hline Ti4 & & 0.000046 & -23.16 & 0.000046 & -45.24 \\
\hline Zr1Ti1Ni1Cu1 & & 0.00695 & -22.15 & 0.00695 & -9.76 \\
\hline
\end{tabular}




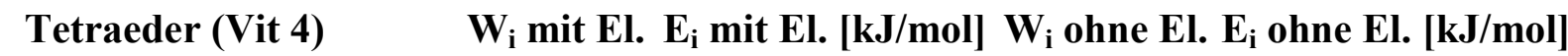

\begin{tabular}{|c|c|c|c|c|c|}
\hline $\mathrm{Zr1Cu} 3$ & & 0.00079 & -17.67 & 0.00079 & 21.9 \\
\hline Zr1Ti2Ni1 & & 0.003822 & -17.61 & 0.003822 & -33.33 \\
\hline $\mathrm{Zr} 2 \mathrm{Cu} 1 \mathrm{Be} 1$ & $*(5)$ & 0.054208 & -16.79 & 0.054208 & -17.96 \\
\hline Zr2Ti1Be1 & $*(4)$ & 0.059629 & -12.81 & 0.059629 & -41.53 \\
\hline $\mathrm{Zr} 2 \mathrm{Ni} 2$ & & 0.013141 & -11.41 & 0.013141 & -21.42 \\
\hline Zr1Ti1Cu2 & & 0.002606 & -9.72 & 0.002606 & -1.67 \\
\hline Zr1Ti2Cu1 & & 0.002867 & -5.60 & 0.002867 & -25.24 \\
\hline Zr1Ti3 & & 0.001051 & -4.81 & 0.001051 & -48.81 \\
\hline Zr2Ti1Ni1 & & 0.021683 & 0.33 & 0.021683 & -36.9 \\
\hline Zr2Ni1Cu1 & & 0.019712 & 0.41 & 0.019712 & -13.33 \\
\hline $\mathrm{Zr} 3 \mathrm{Be} 1$ & $*(1)$ & 0.112754 & 3.85 & 0.112754 & -45.1 \\
\hline Zr2Ti2 & & 0.008944 & 8.67 & 0.008944 & -52.38 \\
\hline $\mathrm{Zr} 2 \mathrm{Ti} 1 \mathrm{Cu} 1$ & & 0.016263 & 11.78 & 0.016263 & -28.81 \\
\hline $\mathrm{Zr} 2 \mathrm{Cu} 2$ & & 0.007392 & 12.21 & 0.007392 & -5.24 \\
\hline Zr3Ni1 & $*(8)$ & 0.041001 & 13.69 & 0.041001 & -40.47 \\
\hline Zr3Til & $*(11)$ & 0.033826 & 18.46 & 0.033826 & -55.95 \\
\hline $\mathrm{Zr} 3 \mathrm{Cu} 1$ & $*(13)$ & 0.030751 & 24.69 & 0.030751 & -32.38 \\
\hline $\mathrm{Zr} 4$ & $*(6)$ & 0.047972 & 25.41 & 0.047972 & -59.52 \\
\hline
\end{tabular}




\subsection{Die Tetraeder in ZANC}

Folgende 35 Tetraeder sind in einer $\mathrm{Zr}_{66,8} \mathrm{Al}_{17,4} \mathrm{Ni}_{7,2} \mathrm{Cu}_{8,8}$-Legierung möglich. Die Wahrscheinlichkeit einen bestimmten Tetraeder in der Legierung $\mathrm{zu}$ finden $\mathrm{W}_{\mathrm{i}}$ (s. Kapitel 2.3.1) und die mittlere Energie $E_{i}$ sind sowohl mit, als auch ohne den Beitrag elastischer Energien berechnet worden (s. Kapitel 2.3.2). Der Einfluss des Wasserstoffblockings (s. Kapitel 2.3.3) ist bereits berücksichtigt. Die 15 häufigsten Tetraeder sind mit einem Stern gekennzeichnet. Die Tetraeder sind aufsteigend nach der mittleren Energie nach Berücksichtigung der elastischen Energien für $\mathrm{S}=0,15$ sortiert.

Tetraeder (ZANC) $\quad W_{i}$ mit El. $E_{i}$ mit El. [kJ/mol] $W_{i}$ ohne El. $E_{i}$ ohne El. [kJ/mol]

\begin{tabular}{|c|c|c|c|c|c|}
\hline $\mathrm{Ni4}$ & & 0.000027 & -60.18 & 0.000027 & 16.68 \\
\hline $\mathrm{Zr} 4$ & $*(2)$ & 0.196506 & -50.49 & 0.196506 & -59.52 \\
\hline $\mathrm{Ni3Cul}$ & & 0.000132 & -49.20 & 0.000132 & 24.77 \\
\hline Zr3Ni1 & *(4) & 0.085001 & -40.54 & 0.085001 & -40.47 \\
\hline Zr1Ni3 & & 0.000994 & -39.83 & 0.000994 & -2.37 \\
\hline $\mathrm{Ni} 2 \mathrm{Cu} 2$ & & 0.000243 & -38.30 & 0.000243 & 32.86 \\
\hline $\mathrm{Zr} 2 \mathrm{Ni} 2$ & $*(14)$ & 0.013788 & -35.76 & 0.013788 & -21.42 \\
\hline Al1Ni3 & & 0.000259 & -34.27 & 0.000259 & 28.32 \\
\hline $\mathrm{Zr} 3 \mathrm{Cu} 1$ & $*(3)$ & 0.104362 & -31.67 & 0.104362 & -32.38 \\
\hline Zr1Ni2Cu1 & & 0.003661 & -30.00 & 0.003661 & 5.72 \\
\hline NilCu3 & & 0.000199 & -27.49 & 0.000199 & 40.95 \\
\hline Zr2Ni1Cu1 & $*(8)$ & 0.033857 & -26.54 & 0.033857 & -13.33 \\
\hline Zr3Al1 & $*(1)$ & 0.205183 & -23.84 & 0.205183 & -28.83 \\
\hline $\mathrm{Al1Ni2Cu1}$ & & 0.000956 & -23.65 & 0.000956 & 36.41 \\
\hline Zr1Ni1Cu2 & & 0.004495 & -20.20 & 0.004495 & 13.81 \\
\hline Zr1Al1Ni2 & & 0.007198 & -18.77 & 0.007198 & 9.27 \\
\hline Zr2Al1Ni1 & $*(7)$ & 0.066566 & -17.40 & 0.066566 & -9.78 \\
\hline $\mathrm{Zr} 2 \mathrm{Cu} 2$ & $*(10)$ & 0.020785 & -17.34 & 0.020785 & -5.24 \\
\hline $\mathrm{Cu} 4$ & & 0.000061 & -16.75 & 0.000061 & 49.04 \\
\hline Al1Ni1Cu2 & & 0.001173 & -13.09 & 0.001173 & 44.5 \\
\hline
\end{tabular}


Tetraeder (ZANC) $\quad$ W $_{\mathrm{i}}$ mit El. E E mit El. [kJ/mol] $W_{i}$ ohne El. $E_{i}$ ohne El. [kJ/mol]

\begin{tabular}{|c|c|c|c|c|c|}
\hline $\mathrm{Zr1Cu} 3$ & & 0.00184 & -10.45 & 0.00184 & 21.9 \\
\hline $\mathrm{A} 12 \mathrm{Ni} 2$ & & 0.00094 & -9.90 & 0.00094 & 39.96 \\
\hline Zr1Al1Ni1Cu1 & $*(11)$ & 0.017676 & -9.11 & 0.017676 & 17.36 \\
\hline $\mathrm{Zr} 2 \mathrm{Al1Cu} 1$ & $*(5)$ & 0.081728 & -8.27 & 0.081728 & -1.69 \\
\hline $\mathrm{A} 11 \mathrm{Cu} 3$ & & 0.00048 & -2.60 & 0.00048 & 52.59 \\
\hline A12Ni1Cul & & 0.002307 & 0.45 & 0.002307 & 48.05 \\
\hline $\mathrm{Zr} 1 \mathrm{~A} 11 \mathrm{Cu} 2$ & $*(15)$ & 0.010851 & 0.51 & 0.010851 & 25.45 \\
\hline Zr2A12 & $*(6)$ & 0.080341 & 0.64 & 0.080341 & 1.86 \\
\hline $\mathrm{Zr} 1 \mathrm{Al} 2 \mathrm{Ni1}$ & $*(12)$ & 0.017376 & 1.61 & 0.017376 & 20.91 \\
\hline $\mathrm{A} 12 \mathrm{Cu} 2$ & & 0.001416 & 10.74 & 0.001416 & 56.14 \\
\hline $\mathrm{Zr} 1 \mathrm{~A} 12 \mathrm{Cu} 1$ & $*(9)$ & 0.021334 & 11.13 & 0.021334 & 29 \\
\hline Al3Nil & & 0.001512 & 13.42 & 0.001512 & 51.6 \\
\hline Zr1Al3 & $*(13)$ & 0.013981 & 21.55 & 0.013981 & 32.55 \\
\hline $\mathrm{A} 13 \mathrm{Cu} 1$ & & 0.001856 & 23.56 & 0.001856 & 59.69 \\
\hline Al4 & & 0.000912 & 36.07 & 0.000912 & 63.24 \\
\hline
\end{tabular}




\section{Literaturverzeichnis}

[Ban98] J. Bankmann, Untersuchungen zum Einsatz der Sekundärionenmassenspektrometrie bei der Tiefenprofilierung dünner Einzel- und Vielfachtschichten, Diplomarbeit, Inst. f. Materialphysik, Göttingen 1998

[Ben87] A. Benninghoven, F. G. Rüdenauer, H. W. Werner, Secondary Ion Mass Spectroscopy, John Wiley \& Sons, 1987

[Bie80] J.P. Biersack, L. Haggmark, Nucl. Instr. and Meth., 174 (1980) 257

[Bro77] Bronstein, Semendjajew, Taschenbuch der Mathematik, Verlag Harri Deutsch, 1977

[Bro89a] R.C. Brouwer, R. Griessen, Phys. Rev. B, 40(3) (1989) 1481-1494

[Bro89b] R.C. Brouwer, J. Rector, N. Koemann, R. Griessen, Phys. Rev. B, 40(6) (1989) 3546-3559

[Cra67] J. Crank, Mathematics of Diffusion, Oxford University Press, 1967

[Dan02] O. Dankert, A. Pundt, Appl. Phys. Lett, 81(9) (2002) 1618-1620

[Dor02] M. Dornheim, Spannungen, Dehnungen und Lage der Phasengrenzen in dünnen Nb- und Y-Schichten bei Wasserstoffbe- und -entladung, Dissertation, Universität Göttingen 2002

[Esh56] J.D. Eshelby, Solid State Physics, eds. F. Seitz und D. Turnbull, Academic, New York, 1956

[Fee88] R. Feenstra, R. Brouwer, R. Griessen, Europhys. Lett., 7(5) (1988) 425-430

[Fla01] T. B. Flanagan, R. Balasubramaniam, R. Kirchheim, Platinum Met. Rev., 15 (2001) 114 und 166

[Flo01] K. M. Flores, R. H. Dauskardt, Acta Mat., 49 (13) (2001) 2527-2537

[Fro76] E. Fromm and E. Gebhardt, Gase und Kohlenstoff in Metallen, Springer Verlag, Berlin (1976)

[Fuk93] Y. Fukai, The Metal-Hydrogen System, Springer-Verlag, Berlin Heidelberg, 
1993

[Ger93] C. Gerthsen, H. Vogel, Physik 17. Auflage, Springer-Verlag Berlin Heidelberg, 1993

[Gey96] U. Geyer, S. Schneider, W.L. Johnson, et al. , Appl. Phys. Lett., 69 (1996) 2492-2494

[Gra66] T. Graham, Phil. Trans. Roy. Soc. London, 156 (1866) 399

[Har87] J. H. Harris, W. A. Curtin, M. A. Tenhover, Phys. Rev. B 36 (1987) 5784-5797

[Ino90] A. Inoue, T. Zhang, T. Masumoto, Mater. Trans., JIM 31 (1990) 425

[Ino92] A. Inoue, T. Nakamura, N. Nishiyama, T. Masumoto, Mater. Trans., JIM 33 (1992) 937

[Ism00] N. Ismail, M. Uhlemann, A. Gebert, J. Eckert, J. Alloys Comp., 198 (2000) 146-152

[Kes00] P. Kesten, Hochauflösende Untersuchung der lokalen Wasserstoffverteilung in metallischen Multischichten mit Hilfe der tomographischen Atomsonde und der Sekundärionenmassenspektrometrie, Dissertation, Universität Göttingen, 2000

[Kie89] W. Kieninger, Löslichkeit und Diffusion von Wasserstoff in amorphen NiZrund NiTi-Legierungen, Dissertation Universität Stuttgart, 1989

[Kim88] J.J. Kim, D.A. Stevenson, J. Non-Cryst. Solids, 101 (1988) 187

[Kir80] R. Kirchheim, Rex B. McLellan, Alloys. J. Electrochem. Soc., 127(11) (1980) 2419-2425

[Kir82] R. Kirchheim, F. Sommer, G. Schluckebier, Acta metall., 30 (1982) 1059-1068

[Kir83] R. Kirchheim, J. Non-Cryst. Solids, 55 (1983) 243

[Kir85] R. Kirchheim, J. Non-Cryst. Solids, 70 (1985) 323-341

[Kir88] R. Kirchheim, Prog. mat. Sci., 32(4) (1988) 262-325

[Mac02] M.P. Macht, S. Mechler, M. Müller, N. Wanderka, Mat. Sci. Forum, 386-388 (2002) 99-104

[Max00] M. Maxelon, Segregation von Wasserstoff und Deuterium an Versetzungen Pd, Dissertation, Universität Göttingen, 2000 
[Nas96] M. Nastasi, J.W. Mayer, J.K. Hirvonen, Ion-Solid Interactions:Fundamentals and Applications, Cambridge University Press, Cambridge, 1996.

[Pek93] A. Peker, W.L. Johnson, Appl. Phys. Lett., 63(17) (1993) 2342

[Pun01] PD Dr. A. Pundt, persönliche Mitteilung

[Ric82] P.M. Richards, Phys Rev B, 27 (1982) 2059

[Sam83]～K. Samwer, W.L. Johnson, Phys. Rev. B, 28(6) (1983) 2907-2913

[Sto84a] U. Stolz, Löslichkeit, Diffusion und partielles Molvolumen von Wasserstoff in amorphen Pd-Si-Legierungen, Dissertation, Universität Stuttgart, 1984

[Sto84b] U. Stolz, R. Kirchheim, J.E. Sadoc, M. Laridjani, J. Less-Comm. Met., 103 (1984) 81

[Suh01]Ｄ. Suh, R.H. Dauskardt, Mat. Trans., 42(4) (2001) 638-641

[Suh99] D. Suh, R.H. Dauskardt, Scripta mater., 42 (2000) 233-240

[Swi78] A. C. Switendick, Theoretical study of hydrogen in metals: current status and further aspects, SAND 78-0250, 1978

[Uch91] H. Uchida, A. Hisano, K. Terao, N. Sato, A. Nagashima, J. Less-Common Met., 172-174 (1991) 1018-1027

[Wan98] N. Wanderka, Q. Wie, R. Doole, M. Jenkins, S. Friedrich, H. Wollenweber, Mat. Sci. Forum, 269-272 (1998) 713

[Wan00]Ｎ. Wanderka, M. P. Macht, M. Seidel, S. Melcher, Appl. Phys. Lett., 77 (2000) 3935-3937

[Web02] Webelements, http://www.webelements.com, 2002

[Win92] H. Windischmann, Critical reviews in solide state and Materials Science, 17(6) (1992) 547

[Yan95] H. W. Yang, S.N. Jenq, Y.Y. Wang, C.C. Wan, J. Alloys Comp., 227 (1995) $69-75$

[Za195] L. Zaluski, A. Zaluska, P. Tessier, J.O. Ström-Olsen, R. Schulz, J. Alloys Comp., 217 (1995) 295-300

[Zan00] D. Zander, U. Köster, N- Eliaz, D. Eliezer, Mat. Sci. Eng., 294-296 (2000) $112-115$

[Zha91] T. Zhang, A. Inoue, T. Masumoto, Mater. Trans., JIM 32 (1991) 1005 
[Zie85] J.F. Ziegler, J.P. Biersack, U. Littmark, The Stopping and Range oflons in Solids, Pergamon Press, New York, 1985. 


\section{Danksagung}

Herrn Prof. Dr. R. Kirchheim danke ich, dass er mir ermöglicht hat, meine Doktorarbeit am Institut für Materialphysik durchzuführen. Vielen Dank auch für die vielen anregenden und konstruktiven Diskussionen.

Ich bedanke mich auch bei meiner Betreuerin Astrid Pundt, die es durch ihr Interesse und ihre Ideen verstand, mich in meiner Arbeit zu motivieren.

Den weiteren Mitgliedern unserer Arbeitsgruppe (auch den ehemaligen) wie Olof Dankert, Kai Nörthemann, Mohammed Suleimann, Martin Dornheim und Christine Borchers danke ich für viele anregende und spannende Gruppensitzungen und Messreisen.

Viele anregende Gespräche habe ich mit Jörg Hoffmann in den letzten Jahren führen können. Die Energie und die Konstruktivität mit der er mir besonders in den letzten Wochen unter die Arme griff, waren unglaublich und unendlich hilfreich.

Eine große Hilfe war die Bereitschaft der Mitglieder der Werkstätten und der Laboranten, alles elektrisch, technisch und feinmechanisch machbare in kürzester Zeit in die Tat umzusetzen. Auch auf Schnitzereien und Gartenpflegetips von unserem Hausmeister musste ich nie lange warten.

Das Leben im Institut machte besonders Spaß durch Chrissi Lang, Lars Kautschor, Torsten Nägel, Jörg Faupel, Sebastian Dreyer und Christian Kluthe. Mit ihnen konnte ich auch häufig noch bis spät in die Nacht über alles denkbare philosophieren.

Christian Schmelcher und unsere für mich sehr wichtigen Diskussionen haben mir außerhalb des Instituts sehr viel geholfen. Dafür und für Tomatix möchte ich ihm herzlichst danken.

Zum Schluss möchte ich meinem Neffen Niklas erwähnen: ohne ein Wort zu sagen, versteht er es mich für alles zukünftige zu motivieren. Meiner Schwester Kerstin und Björn danke ich für ihre tolle Unterstützung aus der Ferne. Vielen Dank an meine Eltern, die mit ihrer Geduld und ihrer Liebe dies alles erst ermöglicht haben. Danke! 


\section{Lebenslauf}

\section{Persönliche Daten}

$\begin{array}{ll}\text { Name: } & \text { Bankmann, Joachim } \\ \text { Geburtsort: } & \text { 49356 Diepholz } \\ \text { Geburtsdatum: } & \text { 01. Oktober 1972 } \\ \text { Eltern: } & \text { Helmut und Gerlinde Bankmann } \\ \text { Nationalität: } & \text { deutsch }\end{array}$

\section{Ausbildung}

$\begin{array}{ll}\text { Schulbildung: } & \text { Allgemeine Hochschulreife am } \\ & \text { Graf-Friedrich-Gymnasium in Diepholz }\end{array}$

Wehrdienst: $\quad 07 / 92-12 / 93$

Studium:

10/93-09/95 Physik-Studium an der
Technischen Universität Clausthal

10/95-10/98 Physik-Diplom-Studium an der

Georg-August-Universität Göttingen

10/98 Physik-Diplomprüfung

seit 06/98 Wissenschaftlicher Mitarbeiter am

Institut für Materialphysik

an der Georg-August-Universität Göttingen 